\title{
The Effect of Age and Gender on the Relative Fatigability of the Human Adductor Pollicis Muscle
}




\title{
The Effect of Age and Gender on the Relative Fatigability of the Human Adductor Pollicis Muscle
}

\author{
By
}

David S. Ditor

\author{
A Thesis \\ Submitted to the School of Graduate Studies \\ in Partial Fulfillment of the Requirements \\ for the Degree \\ Master of Science
}

McMaster University

September, 1999 
Master of Science (1999)

McMaster University

(Kinesiology)

Hamilton, Ontario

Title: $\quad$ The Effect of Age and Gender on the Relative Fatigability of the Human Adductor Pollicis Muscle

Author: David S. Ditor, B.Kin. (University of Western Ontario)

Supervisor: Dr. Audrey L. Hicks

Number of Pages: xiii, 151 


\begin{abstract}
The purpose of this study was to examine the relationships between age, gender and fatigue resistance, and further, to determine the relative influence that estrogen status, membrane excitability, absolute force and muscle length have over the development of fatigue. A total of 48 subjects, classified by age and gender participated in this study; 12 young males ( $25.3 \pm 2.1$ yrs.), 12 young females ( $23.5 \pm 2.1$ yrs.), 12 elderly males ( 71.7 \pm 5.6 yrs.) and 12 elderly females $(69.5 \pm 4.6$ yrs.). The young females were all eumenorrheic, not taking oral contraceptives and tested in the mid-follicular phase of the menstral cycle. None of the elderly females were on hormone replacement therapy.
\end{abstract}

A 3 minute paradigm of intermittent maximal voluntary contractions (MVC) was used to fatigue the adductor pollicis muscle, in which 5s MVC's were alternated with $2 \mathrm{~s}$ rest periods. In addition, maximal twitches were evoked in each $2 \mathrm{~s}$ rest period.

No gender difference in fatigability was evident between young males and young females when considering the fatigue index of the evoked twitch (FI-PT) (young males: $39.8 \pm 26.7 \%$, young females: $36.6 \pm 19.0 \%$ ). There was also no gender difference in fatigability found between elderly males and elderly females when considering the FI-PT (elderly males: $24.9 \pm 26.6 \%$, elderly females: $16.4 \pm 48.9 \%$ ). However, potentiation of the evoked twitches during fatigue may have confounded these measures.

When considering the changes in voluntary force during fatigue, there was a strong trend for a gender by time interaction between young males and young females 
$(p=0.06)$, which suggests that the former were more fatigable. The trend for this gender difference was also apparent in the voluntary fatigue index (FI-MVC) (young males: 44.7 $\pm 10.5 \%$, young females: $37.8 \pm 14.1 \% ; \mathrm{p}=0.12$ ). Similarly, young males had a significant decrease in $\mathrm{M}$-wave amplitude during the fatigue protocol and a trend for a decrease in $M$-wave area $(p=0.08)$, while young females showed no significant decreases in either M-wave measure during the fatigue protocol.

There was no gender difference found between the elderly males and elderly females when considering the FI-MVC (elderly males: $24.2 \pm 10.7 \%$, elderly females: $26.3 \pm 14.5 \%)$. Both groups also showed small but significant reductions in the M-wave amplitude during the fatigue protocol, although $\mathrm{M}$-wave area was well maintained.

The fact that a strong trend for a gender difference in fatigability was found in the young subjects but not the elderly subjects, suggests that estrogen may possess fatigue resisting properties, even during short duration exercise in which glycogen depletion is not a concern.

With respect to the age-related differences in fatigue, elderly males were found to be significantly more fatigue resistant than young males as indicated by the FI-MVC $(\mathrm{p}<0.01)$, and the significant age by time interaction during the fatigue protocol $(\mathrm{p}<0.01)$. In contrast, only a trend was found for an age by time interaction between the young and elderly females during the fatigue protocol $(\mathrm{p}=0.06)$. This trend for an age-related difference in fatigue amongst women was also reflected in the FI- MVC $(\mathrm{p}=0.13)$. 
In conclusion, age was found to be the strongest predictor of fatigability when all subjects were pooled together $(\mathrm{p}<0.01)$, however, gender was also found to play a role in determining fatigue resistance in young adults. In contrast, differences in absolute force and fibre length were not found to contribute to age or gender differences in fatigue, nor were they found to influence fatigability when groups were considered separately. 


\section{Acknowledgements}

I would like to extend my gratitude to the many people who contributed to the completion of this project. First, I would like to sincerely thank Dr. Audrey Hicks for always being approachable and accommodating, well beyond the call of duty, for expressing her confidence in me by encouraging my pursuit of a Ph.D., and for offering her friendship as well as her guidance over the last two years. I would also like to thank my supervisory committee, Dr. Sale and Dr. Galea for cheerfully giving their time and input into this thesis. Special thanks must also go to Dr. Kathleen Martin for her help with the statistical analysis of this work, and to Dr. Stu Phillips for agreeing to sit on the examining committee despite the time restrictions associated with newly found fatherhood. A thousand thank you's to John Moroz for his technical assistance, and for talking me in off the ledge, and to Mary Cleland for running the show. I must also thank Dr. McCartney and Dr. Dowling for hiding the evidence and supplying the get-away car.

There were also many people who helped to make this the most enjoyable academic experience I have ever had. Thanks to my boy Gianni Parise, Eddy the Goose, Timmy the Rose, C3-Pineau, Couller, Jack, Taylor, Thirsty Durk, Elston, Special-K, Snoop Dougy Doug, Golden Boy, Gagme, Saj, Teddy, Hugs, Luc, Ars, the NCAA, the TSE and always Dan Marino and Tony Fernandez. 


\section{Table of Contents}

Abstract

Acknowledgements $\quad$ vi

List of Appendices $\quad \mathrm{X}$

List of Figures $\quad x i$

List of Tables $\quad$ xiii

\section{Chapter I. Introduction}

1.1 Mechanisms of Fatigue 1

1.2 Central Fatigue 1

1.2.1 Excitatory Drive to the Motor Cortex 1

1.2.2 Motoneuron Excitability 3

1.3 Peripheral Neuromuscular Fatigue 4

1.3.1 The Compound Muscle Action Potential 4

1.3.2 The Neuromuscular Junction 6

1.3.3 The Muscle Membrane 7

1.3.4 Excitation-Contraction Coupling 9

1.4 The Effects of Aging on Endurance Performance 10

1.4.1 Age-Related Changes in Skeletal Muscle 10

1.4.2 Age-Related Changes in Fatigue 13

1.4.3 Age, Fatigue and Fibre Type Distribution 14

1.4.4 Age, Fatigue and Membrane Excitability 15

1.4.5 Age, Fatigue and Muscle Blood Flow 18

1.4.6 Age, Fatigue and Metabolism 20

1.5 The Effects of Gender on Endurance Performance 22

1.5.1 Gender Differences in Strength 22

1.5.2 Gender Differences in Fatigue 24

1.5.3 Gender, Fatigue and Absolute Force 25

1.5.4 Gender, Fatigue and Fibre Type Distribution 26

1.5.5 Gender, Fatigue and Muscle Blood Flow 27

1.5.6 Gender, Fatigue and Fibre Length 29

1.5.7 Gender, Fatigue and Metabolism 30 


\section{Chapter II. The Effect of Age and Gender on the Relative Fatigability of the Human Adductor Pollicis Muscle}

$\begin{array}{lll}2.1 & \text { Introduction } & 44\end{array}$

$\begin{array}{lll}2.2 & \text { Methods } & 48\end{array}$

2.2.1 Subjects 48

2.2.2 Stimulating and recording 49

2.2.3 Baseline measures $\quad 50$

2.2.3.1 Evoked twitches $\quad 50$

2.2.3.2 Maximum voluntary contractions $\quad 52$

2.2.4 Fatigue protocol $\quad 52$

2.2 .5 Recovery 53

2.2.6 EMG processing $\quad 54$

2.2.7 Measurement of hand dimensions $\quad 54$

$\begin{array}{lll}2.2 .8 & \text { Statistical analysis } & 55\end{array}$

$\begin{array}{lll}2.3 & \text { Results } & 55\end{array}$

2.3.1 Baseline Measures $\quad 55$

2.3.1.1 Peak twitch torque $\quad 55$

2.3.1.2 Maximum rate of evoked torque development 56

2.3.1.3 Time to peak torque $\quad 56$

2.3.1.4 Half-relaxation time $\quad 56$

2.3.1.5 M-wave Characteristics $\quad 57$

2.3.1.6 Maximal voluntary contractions 58

2.3.1.7 Average voluntary EMG $\quad 58$

2.3.1.8 Motor unit activation $\quad 58$

2.3.1.9 Estimated adductor pollicis length $\quad 58$

$\begin{array}{ll}\text { 2.3.2 Fatigue and recovery measures } & 59\end{array}$

2.3.2.1 The evoked twitch 60

2.3.2.2 Maximum rate of evoked torque development 61 
2.3.2.3 Half-relaxation time $\quad 62$

2.3.2.4 M-wave area $\quad 62$

2.3.2.5 M-wave amplitude $\quad 63$

2.3.2.6 M-wave duration $\quad 64$

2.3.2.7 Maximum voluntary contractions 64

2.3.2.8 AEMG 65

2.3.2.9 Motor unit activation $\quad 65$

2.3.2.10 The AEMG:M-wave area ratio 66

2.3.2.11 Adductor pollicis length and absolute baseline force 66

$\begin{array}{lll}2.4 & \text { Discussion } & 68\end{array}$

2.4.1 Gender differences in fatigue in young adults 68

2.4.2 Gender differences in fatigue in the elderly 73

2.4.3 Age-related differences in fatigue 76

$\begin{array}{ll}2.4 .4 \text { Conclusions } & 78\end{array}$

$\begin{array}{lll}2.5 & \text { Figure Captions } & 84\end{array}$ 


\section{List of Appendices}

Appendix A. Subject Consent Form

Appendix B. The optimal joint angle for adductor pollicis force production in men and women.

Appendix C. ANOVA Tables

123

Appendix D. Raw Data

135 


\section{List of Figures}

Figure 1. Drawing of Apparatus

Figure 2a. Changes in evoked twitch torque, relative to baseline for young males and young females

Figure 2b. Changes in evoked twitch torque, relative to baseline for elderly males and elderly females

Figure 3a. Changes in maximum rate of evoked torque development, relative to baseline for young males and young females

Figure 3b. Changes in maximum rate of evoked torque development, relative to baseline for elderly males and elderly females

Figure 4. Changes in half relaxation time, relative to baseline for males and females

Figure 5a. Changes in M-wave area, relative to baseline for young males and young females

Figure 5b. Changes in M-wave area, relative to baseline for elderly subjects

Figure 6a. Changes in M-wave amplitude, relative to baseline for young males and young females

Figure 6b. Changes in M-wave amplitude, relative to baseline for the elderly

Figure 7. Changes in M-wave duration, relative to baseline for all subjects

Figure 8a. Changes in maximum voluntary torque, relative to baseline for young males and young females

Figure 8b. Changes in maximum voluntary torque, relative to baseline for elderly males and elderly females 
Figure 8c. Changes in maximum voluntary torque, relative to baseline for young males and elderly males

Figure 8d. Changes in maximum voluntary torque, relative to baseline for young females and elderly females

Figure 9. Changes in AEMG, relative to baseline for young and elderly subjects

Figure 10. Changes in percent motor unit activation, relative to baseline for young and elderly subjects

Figure 11. Changes in the AEMG:M-wave area ratio, relative to baseline for young and elderly subjects 


\section{$\underline{\text { List of Tables }}$}

Table 1a. Baseline evoked twitch characteristics

Table 1b. Baseline maximum voluntary torque, AEMG, percent motor unit activation and estimated adductor pollicis length

Table 2. Fatigue indices of the evoked twitch and maximum voluntary contractions 


\section{Chapter 1}

\section{Introduction}

\subsection{Mechanisms of Fatigue}

Although muscle fatigue has been studied extensively, there is much about it that remains to be understood. What makes this subject so elusive is the number of sites along the neuromuscular system which must be considered, as well as the multiple factors within each site which may be susceptible to change during exertion. Complicating the issue further is the fact that the relative contribution of each potential site to fatigue, may depend on the age, gender and fitness level of the individual, amongst other factors.

The mechanisms which may contribute to skeletal muscle fatigue may be classified into two main categories depending on where along the neuromuscular system they are associated. Central mechanisms are those associated with sites proximal to, and including, the motor neurons, while peripheral mechanisms are those associated with sites within the muscle fibres themselves.

\subsection{Central Fatigue}

\subsubsection{Excitatory Drive to the Motor Cortex}

The ability of the central nervous system (CNS) to maximally activate skeletal muscle has been a great source of debate. In a classic study by Merton (1954), it was 
found that in well motivated subjects, the forces produced by maximal voluntary contractions of the adductor pollicis muscle were equal to the forces that were evoked by maximal tetanic stimulation. Furthermore, Merton discovered that the declining force of the adductor pollicis muscle that was apparent during sustained maximal contractions, could not be augmented by either the interpolation of a single supramaximal twitch or a brief train of $50 \mathrm{~Hz}$ tetanic stimulation (Merton, 1954). This absence of CNS failure during muscle fatigue has since been documented many times in the quadriceps, elbow flexors and adductor pollicis muscles (Bigland-Ritchie et al., 1983; Bigland-Ritchie et al., 1986; Gandevia et al., 1998). While these results strongly suggest that the CNS is not a major locus for skeletal muscle fatigue, some exceptions have been noted. For example, Bigland-Ritchie et al. (1986), and Belanger and McComas (1981), have both shown evidence for central failure in the plantar flexor muscles. In the former study, subjects performed intermittent submaximal contractions until a target force could no longer be maintained. At regular intervals, subjects were tested for fatigue by either performing a brief MVC or enduring a brief $50 \mathrm{~Hz}$ tetanic stimulation. Results showed that when only $50 \%$ of the initial MVC could be produced by voluntary effort, the response to $50 \mathrm{~Hz}$ stimulation was still approximately $70 \%$ of that recorded from the unfatigued muscle.

Central fatigue was also found to occur in a more recent experiment by Loscher $e t$ al. (1996a) during sustained submaximal contractions of the plantar flexors. In this study subjects performed contractions at $30 \% \mathrm{MVC}$ until exhaustion. When the endurance limit ( $401 \pm 91$ s) had been reached for voluntary force, the triceps surae was electrically stimulated, and as a result the target force of $30 \% \mathrm{MVC}$ could be maintained for an 
additional 60s. Further, subsequent to this electrical stimulation, subjects could again maintain the required force voluntarily for an additional $85 \pm 48 \mathrm{~s}$. These results suggest that the period of electrical stimulation, although maintaining metabolic stress and contractile fatigue, allowed for a supraspinal, muscle spindle and/or motoneuronal recovery.

More research may therefore be required in order to determine the relative influence of central failure in the loss of force generating capacity during exercise in well motivated subjects.

\subsubsection{Motoneuron Excitability}

It has been frequently observed that the decline in the force generating capacity of a muscle occurs in a parallel manner to the decrease in the muscle's electromyographical (EMG) signal (Bigland-Ritchie et al., 1979; Garland et al., 1988). While this behavior of the EMG activity may be the result of CNS failure, or the loss of excitability at either the neuromuscular junction (NMJ) or muscle membrane, some have argued that it is more likely due to reductions in the excitability of the motoneurons. Support for this hypothesis has been provided by Garland et al. (1988), who noted a decline in the force and EMG activity of the dorsiflexor muscles, after repetitive stimulation of the peroneal nerve, with no accompanying decline in the $\mathrm{M}$-wave and no augmentation of force in response to electrical stimuli interpolated among the voluntary activity. These data refute any notion of transmission block along either the NMJ or muscle membrane, or failure in CNS drive, respectively. Garland therefore concluded that the parallel decline in force 
and EMG were due to a reflex inhibition of motoneurons by afferents from the fatigued muscle. On the other hand, in the previously mentioned study by Loscher et al. (1996a) in which evidence for central fatigue was found, the authors concluded that peripheral inhibition of alpha-motoneurons was of minor significance to the development of fatigue. In addition, further work by Loscher and colleagues (1996b) suggested that the excitatory drive to the triceps surae alpha-motoneuron pool (as indicated by $\mathrm{H}$ reflex measures), actually increased during fatiguing isometric contractions at $30 \% \mathrm{MVC}$.

It is well established that a decline in motorneuron firing rates occurs in parallel with the voluntary fatigue of skeletal muscle force (Grimby et al., 1981; Marsden et al., 1971). While this decline in firing frequency may serve to reduce EMG activity during fatigue, its causative relationship to force reduction, per se, has been challenged. Jones and colleagues (1979), for example, found greater reductions in adductor pollicis force when subjects were stimulated via a constant $80 \mathrm{~Hz}$ frequency, than when they were stimulated with frequencies that were gradually reduced over time. Jones therefore concluded that the reduction in motor neuron firing frequency that occurs during prolonged voluntary effort acts to optimize fatigue resistance rather than hinder it.

\subsection{Peripheral Neuromuscular Fatigue}

\subsubsection{The Compound Muscle Action Potential}

The compound muscle action potential (M-wave) has proven to be an invaluable tool to our understanding of the peripheral mechanisms which contribute to muscle 
fatigue. It is therefore advisable to review the M-wave and the interpretations that may be based on its characteristics, before discussing the mechanisms of peripheral fatigue.

The M-wave is the algebraic sum of all the impulses evoked in a population of muscle fibres, and thus, it is highly representative of the integrity of neuromuscular transmission. Specifically, the M-wave amplitude represents the muscle membrane excitability, as it depends on the resting membrane potential of single muscle fibres, and the size of the action potentials of individual fibres. The M-wave amplitude also gives information regarding the state of neuromuscular transmission, as it is reflective of the number of active muscle fibres. Clearly there are difficulties in interpreting $\mathrm{M}$-wave observations, as changes in amplitude could be caused by either failure at the NMJ or the muscle membrane. Some assistance, however, comes from analysis of the M-wave duration and area. Factors which influence the duration of the M-wave are the synchronization of the muscle fibre action potentials and the conductance of the ionic sodium and potassium channels within the muscle membrane. Therefore, it is agreed that decrements in the M-wave amplitude and area during exertion, despite increases in $\mathrm{M}$ wave duration are indicative of neuromuscular transmission failure, while a maintenance of the M-wave amplitude, during fatigue, accompanied by inreases in $\mathrm{M}$-wave duration and area is representative of a slowing of muscle membrane conduction velocity (Bigland-Ritchie et al., 1979). 


\subsubsection{The Neuromuscular Junction}

There are many discrete sites at the NMJ that may be susceptible to failure during prolonged muscular effort, and similarly, there are many methods which may be used to determine if failure at the NMJ exists. Merton (1954), examined the characteristics of the evoked M-wave as a means of assessing the degree of NMJ failure during maximal voluntary contractions. No reduction of the $\mathrm{M}$-wave amplitude was detected, despite the substantial decline in force that resulted from 3 minutes of maximal adductor pollicis contractions. Merton therefore concluded that muscle fatigue is not associated with failure of transmission across the NMJ. Similar findings of a maintained M-wave amplitude during fatigue have been reported by others in the adductor pollicis (BiglandRitchie et al., 1986) as well as the first dorsal interosseous muscle (Bigland-Ritchie et al., 1982). In contrast, reductions of the $\mathrm{M}$-wave amplitude have been detected during fatiguing contractions of the adductor pollicis muscle (Bellemare and Garzaniti, 1988). However, as stated above, M-wave analysis does not distinguish between the events at the NMJ and those at the muscle membrane, and therefore it may not be a highly reliable test for failure at the NMJ.

More direct methods of measuring NMJ integrity are to a) compare the muscle fatigue produced by indirect nerve stimulation to that produced by direct muscle stimulation, and b) to measure the end plate potentials (EPPS) that result from any given nerve stimulation. Sieck and Prakash (1995), employed the former method and discovered an interesting interaction between the integrity of the NMJ during fatigue and the fibre type of the stimulated muscle. The underlying premise of this study was that 
during repetitive nerve stimulation, the fibres that are more susceptible to fatigue via NMJ failure will not be activated and will not therefore be depleted of glycogen following exhaustive activation. The results revealed that Type IIB muscle fibres exhibited a substantial glycogen depletion as a result of repetitive direct muscle stimulation, whereas repetitive nerve stimulation resulted in far less glycogen depletion in these fibres. In contrast, differences in the depletion of glycogen stores between muscle and nerve stimulation were much less pronounced in the Type I and IIA muscle fibres.

There is also evidence of an interaction between NMJ failure and the stimulation frequency used to produce fatigue. Sieck and Prakash (1995) demonstrated this interaction, in an in vitro rat nerve-diaphragm preparation, by monitoring the EPPS in response to repetitive stimulation at $10,20,40$ and $75 \mathrm{~Hz}$. Failure of the nerve to evoke EPPS were only found during stimulation frequencies of $75 \mathrm{~Hz}$. It was therefore concluded that although NMJ failure can be produced, it is only associated with unphysiologically high stimulation frequencies, and thus, does not contribute to the fatigue of voluntary contractions.

\subsubsection{The Muscle Membrane}

The excitability of the muscle membrane is largely dependent on the electrochemical gradient that exists across the extracellular and intracellular spaces. As indicated by the Goldman-Hodgkin-Katz equation (Hodgkin and Katz, 1949), this electrochemical gradient, or membrane potential, is in turn, greatly determined by the intracellular and extracellular concentrations of sodium $\left(\mathrm{Na}^{+}\right)$and potassium $\left(\mathrm{K}^{+}\right)$. 
During muscle contraction, the inward flux of $\mathrm{Na}^{+}$and the outward flux of $\mathrm{K}^{+}$act to reduce the muscle membrane potential, and it is believed that this membrane depolarization imposes detrimental effects on the amplitude of the muscle action potential. For example, it has been shown that increases in the $\mathrm{K}^{+}$concentrations of a bathing medium from $5 \mathrm{mM}$ to $10 \mathrm{mM}$ cause a $70 \%$ reduction in the muscle action potential in isolated rat diaphragm preparations (Jones, 1981). Likewise, a cell depolarization of 10-20 mV is commonly observed in fatigued muscle cells (Fitts, 1994). It is not clear, however, if these ionically induced changes in membrane potential are sufficient to impede force during voluntary muscle contraction. As mentioned above, the size of the M-wave is commonly maintained despite losses in muscular force (BiglandRitchie et al., 1986; Merton, 1954). Acting in opposition to the ionic fluxes during exertion, and possibly conserving the membrane excitability, is the membrane bound $\mathrm{Na}^{+} / \mathrm{K}^{+}$ATPase. By exchanging $\mathrm{Na}^{+}$and $\mathrm{K}^{+}$across the muscle membrane in an electrogenic manner ( $3 \mathrm{Na}^{+}$out, $2 \mathrm{~K}^{+}$in), the pump has been shown to add approximately $10 \mathrm{mV}$ to the membrane potential at rest, and up to $30 \mathrm{mV}$ during exercise when its activity is enhanced (Hicks and McComas, 1989). However, while the $\mathrm{Na}^{+} / \mathrm{K}^{+}$ATPase may prevent depolarization of the surface membrane, it has been proposed that ionic fluxes may impede muscle excitation at the T-tubules where the pump density is not as great (Fitts, 1994). The exact relationships between membrane depolarization, membrane excitability and muscle fatigue are therefore, still controversial. 


\subsubsection{Excitation-Contraction Coupling}

The role of the T-tubular system is to allow the muscle membrane action potential to propagate into the core of the fibre (Fitts, 1994). When the action potential reaches the T-tubule it is sensed by an intramembranous protein called the dihydropyridine (DHP) receptor. This protein then undergoes a conformational change, which in turn, triggers the release of calcium $\left(\mathrm{Ca}^{2+}\right)$ from adjacent $\mathrm{Ca}^{2+}$ channels in the sarcoplasmic reticulum (SR). The DHP receptor has an essential $\mathrm{Ca}^{2+}$ binding site on its extracellular side, and therefore, the integrity of the T-tubular system is in part, dependent on the extracellular $\mathrm{Ca}^{2+}$ concentrations (Fitts, 1994). Too much T-tubular $\mathrm{Ca}^{2+}$ may induce fatigue by blocking conduction of the action potential, while excessively low $\mathrm{T}$-tubular $\mathrm{Ca}^{2+}$ concentrations may reduce intramembranous $\mathrm{T}$-tubular charge movement and lead to a reduced release of $\mathrm{Ca}^{2+}$ from the SR (Fitts, 1994). For example, Howell and Snowdowne (1981), showed a linear fall in peak tension as extracellular $\mathrm{Ca}^{2+}$ concentrations rose from 1-20 mM, and concluded that this force decline was a result of $\mathrm{C} \mathrm{Ca}^{2+}$ induced conduction block in the T-tubule.

Aside from the T-tubular conduction block, failure of excitation-contraction coupling may result from a depletion of releasable $\mathrm{Ca}^{2+}$ from the SR. This is unlikely to be a major factor in fatigue, however, as Eberstein and Sandow (1963), were able to reverse the tension loss in fatigued muscle fibres with the administration of caffeine, a known stimulator of $\mathrm{Ca}^{2+}$ release from SR stores. 
Finally, metabolic factors may impede the excitation contraction coupling as reductions in $\mathrm{pH}$ have been found to reduce the frequency and duration of ryanodine channel opening (Fitts, 1994).

\subsection{The Effects of Aging on Endurance Performance}

\subsubsection{Age-related Changes in Skeletal Muscle}

Perhaps the easiest to detect, and most widely reported, age-related change in skeletal muscle is its diminished force generating capacity. This loss of strength has been demonstrated in a variety of muscles, regardless of whether the force is generated voluntarily or via electrical stimulation. For example, the twitch and tetanic tensions of the triceps surae, as well as maximal voluntary contractions, have been shown to be $33 \%$, $49 \%$ and $43 \%$ lower respectively, in elderly males as compared to young males (Davies et al., 1983). Likewise, Winegard et al. showed a $26.5 \%$ decrease in voluntary plantarflexor strength in females when comparing 60-80 year old to $20-30$ year old women (Winegard et al., 1997). Similar age-related decrements in strength have been found in both large muscle groups such as the leg extensors (Hakkinen and Hakkinen, 1991) and elbow flexors (Hicks and McCartney, 1996), as well as the smaller intrinsic hand muscles such as the adductor pollicis (Bruce et al., 1989; Narici et al., 1991). While some reduction in muscle mass and strength has been shown to occur in the fifth and sixth decades of life, it is generally agreed that significant reductions in force generating 
capacity are not evident until the age of 50-60 years (Govindasamy and Paterson, 1994; Hakkinen and Hakkinen, 1991; Lexell et al., 1988).

The decline in strength with age has been attributed, in part, to both a loss of Type I and Type II fibres, as well as a selective atrophy of Type II fibres (Lexell et al., 1988). These changes in fibre size and number appear to be neuropathic in origin as opposed to myopathic. Fewer motor neurons are found in the spinal cord of the elderly (Tomlinson and Irving, 1977), and further, aged muscle exhibits a reduction in the number of functioning motor units with an increase in the size of the surviving low-threshold motor units (Brown, 1972; Stalberg and Fawcett, 1982). Together, these observations suggest an age-related process of denervation and subsequent reinnervation, via collateral sprouting, of skeletal muscle which results in fibre type grouping and a gradual loss of strength. The process of denervation/reinnervation with aging however, may be specific to certain muscles. For example, Galea (1996), found no significant loss of motor units in elderly biceps muscles, and although both the thenar and EDB muscles were found to experience denervation with aging, only the former showed evidence of subsequent reinnervation. In contrast however, Doherty and colleagues (1993) found evidence for both a loss of motor units and collateral reinnervation in the biceps brachii and brachialis muscles of elderly men and women aged 60-81 years.

While age-related muscle atrophy is well established, it is still unclear as to whether there is a reduction in the specific tension (force/CSA) of the surviving muscle. Hakkinen et al. (1991), found no change in MVC force/CSA with age in the quadriceps femoris muscle, while Bruce and colleagues (1989), did find a specific tension reduction 
in the elderly adductor pollicis muscle. However, in the latter study the age-related changes in body composition were not accounted for and therefore the CSA of the muscle per se may have been overestimated in the elderly. Brookes and Faulkner (1988), examined the EDL and soleus muscles of male mice and found that maximal force normalized for total fibre CSA was $20 \%$ lower for aged mice ( 28 months) as compared to young ( 3 months) and adult (12 months) mice. These researchers later discovered that single permeabilized muscle fibres from aged mice, when maximally activated by a high calcium concentration, develop the same maximal specific forces as fibres from adult mice (Brookes and Faulkner, 1994). This observation suggests that the factors responsible for possible age-related declines in specific force may be "upstream" from the actual myofilaments.

More research is needed to settle the issue of age and its effect on specific tension, however, some interesting observations have been made which point toward an ageassociated decrease in specific tension. For example, evidence of deficits at the neuromuscular junction have been found in aged rats, including a reduction in the number of nerve terminals (Tucek and Gutmann, 1973), as well as a decreased concentration of acetylcholine (Smith and Weiler, 1987). Furthermore, work by Larsson and Salviati (1989), suggests that the volume of the sarcoplasmic reticulum may decrease with age in Type II rat skeletal muscle fibres. 


\subsubsection{Age-related Changes in Fatigue}

The age-related loss of muscle mass and strength dictates that at any absolute load the elderly should fatigue more quickly than the young. Some of the earlier work examining aging and fatigue supports this contention (Burke et al., 1953; Collumbine et al., 1950), and while such findings have practical implications, it is of greater interest to investigate the age-related changes in relative fatigability. Despite the extensive amount of research in this area there is still no clear consensus as to the relative fatigue resistance in elderly compared to young adults. Complicating the issue are several potentially confounding factors that accompany the aging process, including the general decline in physical activity (Govindasamy and Paterson, 1994), as well as subtle changes in the response of elderly muscle to electrical stimulation. For example, Cupido et al. in 1992, measured the decline in torque during $60 \mathrm{~s}$ of tetanic stimulation of the dorsiflexors and found the elderly to be less (at $20 \mathrm{~Hz}$ ) or equally resistant (at 30 and $40 \mathrm{~Hz}$ ) to fatigue as compared to the young. However, these findings must be interpreted carefully, with consideration given to the age-related changes in the force-frequency relationship. Specifically, it has been shown that, with aging, comes a leftward shift in the forcefrequency curve (Narici et al., 1991). Therefore, at any given stimulation frequency up to approximately $50 \mathrm{~Hz}$ (as was the case in the Cupido study), the elderly will produce contractions at a higher percentage of their maximal tetanic force, as compared to the young, and thus, show an underestimated resistance to fatigue. 


\subsubsection{Age, Fatigue and Fibre Type Distribution}

As previously stated, there is an age-associated process of muscular denervation and reinnervation via collateral sprouting that results in a reduction in the number of both Type I and Type II fibres, as well as a selective atrophy of Type II fibres (Lexell et al., 1988). Although such changes in the elderly are well established, the resulting net effect on fibre type distribution remains controversial.

It is reasonable to assume that any age-related changes in muscle which favour an eventual dominance in Type I fibre representation, would confer benefits to the relative fatigue resistance in the elderly. This follows not only from the greater endurance capabilities of slow-twitch as compared to fast-twitch fibres, but also from the possibility that Type I fibres could serve as a recipient for the lactate produced in Type II fibres (Tesch et al., 1978).

Larsson and Karlsson (1978), examined biopsies from the vastus lateralis muscle in 50 male subjects between the ages of 22 and 65 years, all of whom were reported as sedentary. Their results showed that the percentage of both Type IIA and Type IIB muscle fibres decreased with age, as did Type II fibre area. Furthermore, the area of Type I fibres was found to be unaffected by age, and consequently, the Type II/I fibre area ratio decreased from 1.30 to 0.99 in the $20-29$ and 60-65 year old groups, respectively. Isometric and dynamic endurance tests were also performed in this study; the former being defined as the length of time that a force equal to $50 \%$ of the MVC could be maintained, and the latter being defined as the relative decline in peak torque that resulted from 50 maximal isokinetic contractions of the quadriceps. Both isometric and dynamic 
endurance showed significant negative correlations to Type II fibre area. Further, the ability to perform these fatigue protocols tended to increase in the elderly, although not to significant levels.

In contrast, studies examining the vastus lateralis (Essen-Gustavsson and Borges, 1986), and the gastrocnemius muscles (Coggan et al.,1992), in young and old men and women, found no age-related change in the fibre type distribution in either gender. However, while Essen-Gustavsson and colleagues found atrophied Type I and Type II fibres in the elderly with no apparent age-related change in the Type II/Type I fibre area ratio, the Coggan study found age-related atrophy in only the Type II fibres, and a significant decrease in the Type IIa/TypeI and Type IIb/Type I fibre area ratios.

Such discrepancies in the literature may be due to the biopsy procedure which may have questionable worth in fibre type distribution assessment (Essen-Gustavsson and Borges, 1986; Lexell et al., 1988), and a tighter consensus may result from more appropriate techniques. For example, when cross-sections of whole vastus lateralis muscles were examined (in 43 previously healthy men, aged 15-83 years, 3 days post mortem), there was no age-related change found for fibre type distribution (\% Type I fibres), and while the Type II fibres atrophied significantly with age, there was no such decrease in the area of Type I fibres (Lexell et al., 1988).

\subsubsection{Age, Fatigue and Membrane Excitability}

The excitability of the muscle membrane, at rest, has been reported to decrease with age (Campbell et al., 1973; Vandervoort and McComas, 1986). This degeneration 
has been attributed to age-related decreases in both chloride conductance and the activity of the $\mathrm{Na}^{+} / \mathrm{K}^{+}$ATPase enzyme, which together, act to decrease the resting membrane potential in aged muscle (De Luca et al., 1990; Kjeldson, 1987). For example, Frolkis et al. (1976) observed a significant 6-9 $\mathrm{mV}$ decrease in the resting membrane potential of old rats. Experimentally, the muscle membrane excitability can be evaluated by measurement of the compound muscle action potential (M-wave). Specifically, the peakto-peak amplitude of the M-wave is symbolic of the muscle membrane excitability as it is dependent on both the resting membrane potential and the extent of the action potential overshoot in single muscle fibres (Milner-Brown and Miller, 1986).

The maintenance of muscle membrane excitabilty during muscle fatigue is still under some debate, as some studies have reported no change in the M-wave amplitude during muscular exertion (Bigland-Ritchie et al., 1986; Merton, 1954), while others have noted a significant decrease (Cupido et al., 1992). The lack of consensus on this issue, however, may be due to the variety of chosen fatigue protocols between studies. For example, some of those who have reported no significant decline in the M-wave during exercise have employed a voluntary fatigue model (Bigland-Ritchie et al., 1982), while those who have noted $\mathrm{M}$-wave reductions have utilized electrical stimulation to induce fatigue (Bigland-Ritchie et al., 1979; Cupido et al., 1992). Because there is still some debate as to whether muscle membrane excitability decreases during exercise, it is difficult to answer with any confidence if this excitability during exercise exhibits an agerelated difference. Nevertheless, two studies in particular have shed some light on this aging issue. 
Cupido et al. (1992), stimulated the tibialis anterior muscles of elderly (67.7 years) and young ( 26.7 years) adults at frequencies of 20,30 , or $40 \mathrm{~Hz}$ for $60 \mathrm{~s}$. The Mwave amplitudes were found to decrease during exercise in each group, and while the size of the M-wave was consistently depressed in the elderly as compared to the young, there was no age-related difference in the relative amount of $\mathrm{M}$-wave reduction over the 60 second fatigue protocol. However, as mentioned previously, this study did not account for the age-related change in the force frequency curve and therefore, the elderly subjects may have produced greater relative forces during their fatigue protocol than the young.

Hicks et al. (1992), also studied the effects of aging on muscle membrane excitability during fatigue. Hicks utilized a 2 minute voluntary fatigue model in the brachioradialis, tibialis anterior and thenar muscles of elderly (66.3 years) and young (31.2 years) adults. Furthermore, the subjects in this experiment performed these fatigue protocols before and after 12 weeks of resistance training. The results showed that although the M-waves appeared to be consistently smaller in the elderly adults, as compared to the young during the 2 minute test, there was no significant fatigue-related reduction in the M-wave amplitudes in either group. As well, the muscle membrane excitability was shown to increase in elderly muscle following the 12 weeks of resistance training (although it was still depressed compared to the young), and it remained resistant to reductions during the 2 minute fatigue protocol. It should also be noted, that since the M-wave amplitude is a summed response of the many muscle fibre action potentials, that the age-related decline in this measure may be accounted for, in part, by the age- 
associated loss of muscle fibres, rather than a loss of membrane excitability per se. Hicks therefore demonstrated that although there are inevitable reductions in muscle membrane excitability as a result of aging, the maintenance of this excitability during exercise is a property that persists in the skeletal muscles of the elderly.

\subsubsection{Age, Fatigue and Muscle Blood Flow}

The blood flow to exercising skeletal muscle would seem to have great influence over the muscle's ability to resist fatigue. The capilliarity not only contributes to the degree of oxygen delivery, but also to the clearance of muscle metabolites and therefore, plays an important role in the muscle's ability to resist fatigue during both aerobic exercise and more intense anaerobic exercise. It is therefore of interest to determine if there are any age-related changes in skeletal muscle capilliarity, and if so, the relative influence that these changes bear on skeletal muscle fatigue.

Irion and colleagues (1987) addressed these questions by examining the fatigability and muscle blood flow in young and old rats during maximal intermittent tetanic contractions of the plantar flexors. The senescent rats exhibited both a greater degree of fatigue following 10 minutes of stimulation as well as a reduced blood flow to the Type I, IIa, and IIb fibres of the exercising muscles. Further, no age-related differences were found for exercising heart rate or mean arterial pressure. The reduction in muscle blood flow in the senescent rats was in part, accounted for by their inability to dilate the vessels in their skeletal muscle. Although it was the absolute force decline that was measured in this study, there was not a significant difference in the baseline tetanic 
strength between the young and old rats. It would therefore appear that the aged rats had a greater relative fatigability, possibly due to reductions in muscular blood flow.

However, it is also interesting to note that the senescent rats had a significantly smaller plantar flexor muscle mass than the young, and therefore, in light of their similar strength, could have had greater specific strength. This force/CSA advantage in the elderly rats may have contributed to the age-related reduction in muscle blood flow via a greater relative pressure being exerted on the vessels. While this age-related advantage in specific tension is commonly found in rats (Eddinger et al., 1986; Fitts et al., 1984), the force/CSA is generally believed to remain unchanged (Hakkinen and Hakkinen, 1991) or decline (Bruce et al.,1989) with age in humans, and therefore, the results of the Irion study may not be generalizable to the human model.

The studies that have investigated the possibility of age-related changes in human skeletal muscle capillarization have produced conflicting results. There are studies which suggest that capillarization does not decrease with age (Denis et al., 1986; Jakobsson et al., 1990), however these researchers have been criticized for comparing physically active elderly people to young sedentary controls (Coggan et al., 1992). In a more rigorously controlled study, capillary density was found to decline by approximately $25 \%$ between the ages of 20 and 69 years in the gastrocnemius muscle (Coggan et al., 1992).

Measures of capillarization by themselves, however, may not be sufficient in determining the skeletal muscle blood flow during exercise. This point is emphasized by Haidet and Parsons (1991), who found no age-related decline in skeletal muscle blood flow during maximal exercise in beagles, despite a significant reduction in the 
capillary/fibre ratio of the exercising muscles. This finding was explained by the ability of the senescent beagles to adequately redirect splanchnic blood flow to the exercising limbs.

\subsubsection{Age, Fatigue and Metabolism}

The ability to derive energy from fuel sources clearly has great influence over an individual's ability to perform prolonged exercise. Substrate metabolism during exercise is an extremely broad topic, much of which is beyond the scope of this review. Due to the exercise protocol chosen in the present study, the age-related changes in glucose metabolism during short duration exercise will be the main focus of this section.

In a study conducted by Dudley and Fleck (1984), the plantar flexors of young and old rats were stimulated for 3 minutes at either $2 \mathrm{~Hz}$ (45 tetani/min) or $5 \mathrm{~Hz}$ (90 tetani/min). The results showed that the ATP and phosphocreatine levels of the aged rats were significantly more depressed in nearly all the stimulation trials as compared to the young. Further, the lactate levels were significantly higher in the aged rat muscle as compared to the young. The initial resting levels of lactate, ATP and phosphocreatine could not have been confounding factors as they were similar in the young and old; a finding in agreement with some researchers (Fitts et al., 1984; Taylor et al., 1984), and in conflict with others (Ermini and Verzar, 1968). It is also unlikely that Dudley's results were due to age-related differences in resting muscle glycogen (Cartee and Farrar, 1988; Fitts et al., 1984). 
Similar results were found by Fitts and colleagues in 1984. Specifically, Fitts found that following 30 minutes of electrical stimulation of the soleus, elderly rats showed a significantly greater glycogen and phosphocreatine depletion, and an exaggerated muscle lactate accumulation than young rats. These age-related differences were found despite equal resting concentrations of glycogen, lactate and phosphocreatine, between the two age groups. These results suggest that the glycolytic system was stressed to a greater degree in the elderly rats despite a common maximal workload. The mechanisms accounting for these age-related differences, however, are not clear. Studies investigating the activity of various glycolytic enzymes have failed to explain the greater glycolytic stress in the elderly during exercise. In a review by Cartee (1994), it was stated that the maximal activities of phosphorylase and phosphofructokinase (PFK) are not altered in elderly rat muscle, although lactate dehydrogenase (LDH) activity has been reported to be lower (23\%). It has been hypothesized that there are age-related deficits in aerobic metabolism, and that these deficits necessitate greater demands on glycolysis during exercise in the elderly. This theory is supported by findings of reduced activities of citrate synthase and succinate dehydrogenase in the exercising muscles of the aged rat (Holloszy et al., 1991).

Human studies also provide some support for the hypothesis of an age-related increase in glycolysis dependence during exercise. For example, Essen-Gustavsson and Borges (1986), found a significant age-related decrease in citrate synthase activity, with no concomitant decrease in the activities of glycolytic enzymes such as LDH or hexokinase (HK). Likewise, Coggan et al. (1992), found no age-related decrease in 
phosphorylase, PFK or LDH activity, while the activities of mitochondrial enzymes such as succinate dehydrogenase and citrate synthase were found to decline by approximately $25 \%$ between the ages of 20-69 years. While greater degrees of energy depletion may be expected to result from these age-related changes in enzyme activities, there is evidence in human studies which suggests otherwise. For example, in a study conducted by Taylor and colleagues in 1984, there were no age-related differences in phosphocreatine depletion or the fall in muscle $\mathrm{pH}$ following 5 minutes of handgrip exercise.

\subsection{The Effects of Gender on Endurance Performance}

\subsubsection{Gender Differences in Strength}

The male advantage in absolute strength is well documented (for a detailed review see Laubach, 1976; Miller et al., 1993). This advantage is emphasized in the upper limbs, as women have been shown to have $60-80 \%$ of men's leg strength, but only $50-60 \%$ of men's upper limb strength (Sale, 1999). This gender difference in absolute strength has been attributed to a greater muscle size in men. To expand, the female thigh muscle cross-sectional area (CSA) is generally $65-70 \%$ of that in men, and the female upper arm muscle CSA is generally $50-60 \%$ of that in men (Sale, 1999). These gender differences in strength are apparent in both isometric (Miller et al., 1993), or dynamic (Sale et al., 1987), testing protocols. While the ability to fully activate the muscle is also of vital importance to voluntary strength measures, studies have shown no gender differences in this variable (Miller et al., 1993). 
Relative strength is commonly expressed as either strength per unit body mass, or strength per unit lean body mass (fat-free mass). Because men, in general, have a greater body mass than women, as well as a lower percentage of body fat (Maughan et al., 1983; Miller et al., 1993), the gender differences in strength are not as pronounced when expressed in these relative terms. For example, Miller and colleagues (1993), found the absolute knee extension strength in women to be $69 \%$ of that in men, while their knee extension strength expressed per unit of lean body mass was $80 \%$ of that in men.

While it is established that males have a greater quantity of muscle than females, it is still debatable whether males also have a greater quality of muscle. Muscle quality, or specific tension, refers to the strength per unit of muscle cross-sectional area. A review of the literature by Sale (1999), expresses the general agreement that there is no significant gender difference in the specific tension of human skeletal muscle, although some exceptions have been noted in the knee extensors and flexors (Kanehisa et al., 1994; Young et al., 1985). Any observed gender difference in muscle quality have been attributed to the greater relative amount of intramuscular fat (Kanehisa et al., 1994) and connective tissue (Miller et al., 1993) that females possess as compared to males. The smaller relative area of Type II fibres in female muscle (to be discussed)(Miller et al., 1993), may also account for any observed gender differences in specific force as Type II muscle fibres have been reported to produce 1.8 times greater forces per CSA than Type I fibres (Grindrod et al., 1987; Young et al., 1984). 


\subsubsection{Gender Differences in Fatigue}

Because of the distinct gender difference in strength, any absolute load will represent a lower percentage of the maximum force generating capacity for males as compared to females, and therefore, men also show superior absolute endurance (Maughan et al., 1986). While the ability to maintain any given absolute force may have practical, or performance related significance, it may be of greater theoretical interest to study the possible gender differences in relative endurance.

When endurance tasks are performed relative to one's own strength, women commonly exhibit a greater resistance to fatigue than their age-matched male counterparts. This gender difference in fatigability has been demonstrated over a wide range of muscles, and seems to persist despite the aging process. For example, in young subjects, West and colleagues (1995), discovered a significant female advantage in enduring sustained, submaximal handgrip contractions, while Maughan et al. (1986), detected a similar relative female fatigue resistance during sustained submaximal quadriceps contractions, as well as during dynamic submaximal elbow flexor exercise. Furthermore, Hicks and McCartney (1996), found elderly women to be less fatigable than elderly men during ankle dorsiflexor contractions. The exact mechanisms underlying this apparent gender difference in relative endurance remain to be elucidated, although several theories have been proposed. 


\subsubsection{Gender, Fatigue and Absolute Force}

As stated above, the male advantage in absolute force production, allows men to be more fatigue resistant than women when both sexes are asked to maintain any given absolute load. In contrast, it has been suggested that this male strength advantage may be responsible, in part, for the greater fatigability in men as compared to women during endurance tasks at any given relative load. The premise behind this suggestion is that the lower absolute force production in females during relative endurance tasks may translate to less local muscle ischemia and therefore a greater fatigue resistance compared to males.

Lewis and colleagues (1997), tested this hypothesis with a unique protocol that matched men and women for absolute adductor pollicis strength. Subjects performed intermittent isometric contractions ( $5 \mathrm{~s}$ contraction, $5 \mathrm{~s}$ rest) of thumb adduction at $50 \%$ pre-exercise MVC until exhaustion, i.e. 50\% of the original MVC force could no longer be maintained for $5 \mathrm{~s}$. Lewis found that despite the matched adductor pollicis strength, women remained decisively less fatigable than men. Specifically, women were able to produce the required forces for $14.1 \pm 1.2 \mathrm{~min}$. while men could not continue past $7.9 \pm$ $0.7 \mathrm{~min}$.

While the results of this study suggest that the lower absolute force generating capacity in females is not influential to the gender difference in fatigue, it must be noted that Lewis failed to include any EMG or motor unit activation measurements in his protocol, and therefore, possible gender differences in motivation or muscle activation could have confounded Lewis' findings. 


\subsubsection{Gender, Fatigue and Fibre Type}

It has been hypothesized that the gender difference in fatigue resistance is, in part, accounted for by females possessing a greater relative amount of fatigue resistant Type I

muscle fibres than males. As mentioned previously, females would benefit not only from the greater fatigue resistance of slow-twitch fibres, but also from the possibility that Type I fibres could serve as a recipient for the lactate produced in Type II fibres (Tesch et al., 1978). There is evidence to support such a theory as women have been reported to have greater Type I/II area ratios (Nygaard, 1981; Simoneau and Bouchard, 1989), and a greater percentage of Type I fibres (Miller et al., 1993; Simoneau et al., 1985) than men. Various studies, however, have challenged the theory of a Type I dominance in females as compared to males. Specifically, Sale et al. (1987) found no gender difference in either the percentage of Type II fibres or the percent Type II fibre area in the biceps, and Schantz et al. (1983), found no gender differences in the percentage of Type I, IIA, or IIB fibres in the vastus lateralis.

The theory that the gender difference in fatigue resistance is accounted for by a gender difference in fibre type is subject to further criticism, as there is even controversy regarding the correlation between isometric endurance and fibre type distribution. For example, Maughan and colleagues (1985), found no correlation between the percentage of Type I fibres or the percent Type I fibre area and knee extension endurance at 20, 50 and $80 \%$ of the MVC. 


\subsubsection{Gender, Fatigue and Muscle Blood Flow}

As stated previously, the ability to adequately supply blood to exercising muscle is vitally important to the muscle's ability to endure exercise. Thus, any gender differences in skeletal muscle blood flow could account for a large portion of the gender difference in fatigability. In healthy individuals, sufficient muscular blood flow during exercise is, in the most part, due to the muscle capillarization, which can be expressed as either the number of capillaries per fibre, or the number of capillaries per unit of muscle CSA. Bell and Jacobs (1990), compared these two indices of muscular blood flow between males and females, and found no significant gender difference in either measure. Furthermore, no gender differences in capillarization were detected between male and female body builders, suggesting a strong and persistent gender similarity.

Although there may be no gender difference in capillarization, there still may be muscular differences between men and women, which create a female advantage in muscle blood flow. For example, although the majority of literature suggests otherwise (Sale, 1999), there is some evidence to support the case of a male advantage in specific tension (Kanehisa et al., 1994; Young et al., 1985). Gender differences in the force per unit CSA of muscle may contribute to the gender difference in fatigability because, as explained by Sale (1999), in isometric endurance tasks where blood flow is compromised, the lower intramuscular force per CSA in female muscle may make women less susceptible to occlusion of blood flow. A study by West and colleagues (1995), allows this theory to be examined in some detail. In West's experiment, male and female subjects performed sustained isometric handgrip contractions at 30,50 and $75 \%$ 
of their pre-exercise MVC. The results showed women to be significantly more fatigue resistant at each of the exercise intensities as measured by time to fatigue (women vs. men; $400.7 \pm 35.8$ vs. $364.3 \pm 34.4$ s, $205.1 \pm 15.6$ vs. $139.4 \pm 13 \mathrm{~s}$, and $89.9 \pm 11.4$ vs. $66.4 \pm 6.4 \mathrm{~s}$ at $30 \%, 50 \%$ and $75 \% \mathrm{MVC}$, respectively). The fact that women showed a greater fatigue resistance than men during the most intense contraction trial (in which a complete occlusion of blood would be expected in both sexes), suggests that gender differences in fatigue are independent of blood flow. However, it should be noted that the female endurance advantage may have been more pronounced at the lower intensities, in which some blood flow would be expected. Although West did not statistically report on these relationships, it appears that women lasted $135 \%$ as long as men at $30 \% \mathrm{MVC}$ but only $110 \%$ as long as men at $75 \%$ MVC. Similarly, in a study by Maughan et al. (1986) women were found to be more fatigue resistant than men during sustained isometric contractions at $20 \% \mathrm{MVC}$ (when some blood flow would be expected), but no gender differences in fatigue were found during the higher intensities of 50 and $80 \%$ MVC in which subjects likely experienced an occlusion of muscle blood flow. It may be argued, therefore, that gender differences in blood flow, secondary to gender differences in specific force may contribute to the female advantage in fatigue resistance. The studies by West and Maughan raise some interesting questions that warrant further investigation.

Finally, it has also been suggested that there is a gender difference in vascular reactivity that may result in a female advantage in muscular blood flow. To expand, a study by Li and colleagues (1997), concluded that vasoconstriction is less in females, as 
submaximal norepinephrine concentrations produced less constriction of female rat tail arteries than that in male rat tail arteries. Li however, did not use an exercise model to test this theory, and therefore, did not account for the complex relationships between vasoconstriction, vasodilation, and blood redistribution during exercise. Li's conclusions, therefore, may be misleading. For example, the greater vasoconstriction in male rat arteries, if restricted to non-exercising vessels, may enhance blood redistribution and actually increase the blood flow to exercising muscles.

\subsubsection{Gender, Fatigue and Fibre Length}

de Haan and colleagues (1988), introduced the idea that gender differences in muscle dimensions may, in part, account for the observed gender difference in fatigue resistance. It was de Haan's contention that, although the maximal force generating capacity of a muscle is determined by its cross-sectional area, it is the total muscle volume that determines the energy cost of a contraction. Therefore, for any two muscles of equal CSA, but different lengths, the metabolic demand of any given force will be greater for the longer muscle (assuming a constant mean sarcomere length between muscles). This is because the number of sarcomeres in series increases the energy utilization of a contraction, but does not enhance force production. Since males are, in general, larger than females and thus have longer muscles, the hypothesis of de Haan becomes relevant in the discussion of gender differences in fatigue. To expand, the fewer number of sarcomeres in series in female muscle allows for more economical relative 
force production, which in turn, translates to a greater fatigue resistance in women. The de Haan theory, however, has yet to be adequately tested.

\subsubsection{Gender, Fatigue and Metabolism}

As outlined above, the ability to resist fatigue during exercise is greatly influenced by the ability to efficiently derive energy from fuel sources. A complete detailing of the gender differences in substrate metabolism reach well beyond the scope of this discussion (for an excellent review see Tarnopolsky, 1999). Due to the exercise protocol chosen in the present study, the gender differences in glucose metabolism during exercise, will be the main focus of this section.

It has been hypothesized for some time that females rely less on carbohydrate (CHO) metabolism during exercise than males, and while some early work has supported this notion, hard conclusions have been difficult to obtain due to the numerous variables that must be controlled. For example, in a relatively early study by Costill et al. (1979), trained male and female subjects performed 60 minutes of treadmill exercise at $70 \%$ $\mathrm{VO}_{2 \max }$, and blood and expired air samples were analyzed in order to determine the relative contributions of $\mathrm{CHO}$ and fat metabolism. Costill found no gender differences in the respiratory exchange ratio (RER), and a higher blood lactate concentration in females after 60 minutes, however, these conclusions have been highly criticized as Costill neglected to account for individual differences in lactate thresholds as well as the menstral cycle stage of the female subjects. This lack of control for variables such as 
fitness levels, menstral phase and diet were commonplace in the early investigations in this area (for a review see Ruby, 1999).

In the last decade however, rigorously controlled experiments by Tarnopolsky et al. (1990, 1995), have helped to clarify the gender differences in CHO metabolism during exercise. In the first of these experiments (Tarnopolsky et al., 1990), males and females were matched for various aspects of fitness and activity, and females were eumenorrheic, not currently taking oral contraceptives and tested during their midfollicular phase. Females were found to have significantly lower RER values throughout the $15 \mathrm{Km}$ treadmill exercise protocol (approx. $63 \%$ of $\mathrm{VO}_{2 \max }$ ) as compared to males, and further, females showed a lesser degree of muscle glycogen depletion than males. These results strongly suggest a lower reliance on $\mathrm{CHO}$ metabolism during exercise in females. In a later experiment Tarnopolsky et al. (1995), exercised rigourosly matched males and females after 5 days of either a high ( $75 \%)$ or "low" $(\sim 59 \%)$ CHO diet. Despite a higher exercise intensity ( $75 \% \mathrm{VO} 2 \mathrm{max}$ for $60 \mathrm{~min}$. followed by $85 \% \mathrm{VO} 2 \mathrm{max}$ to exhaustion) than in the previous study, women were again found to have lower RER values throughout exercise, although there was no gender difference in muscle glycogen depletion. It was therefore concluded that, regardless of diet, males exhibit a greater dependence on $\mathrm{CHO}$ metabolism during exercise than females.

It must be noted, however, that each of the above experiments examined the gender differences in metabolism during exercise of relatively long duration. The literature is much more scarce as to the gender differences in $\mathrm{CHO}$ utilization during relatively short duration exercise, where the energy supplied by anaerobic glycolysis is of 
greater importance. Nevertheless, one study in particular, conducted by Jacobs et al. (1982) examined the lactate accumulations of female subjects during 30s Wingate tests. Although no male subjects were included in this experiment, Jacobs and colleagues reported distinctly lower post-exercise lactate levels in their female subjects as compared to the typical lactate levels found in males during similar exercise protocols. However, gender differences in workload during the Wingate test, rather than differences in glycolytic capacity per se, may be responsible for these apparent gender differences in lactate accumulation. Further insight, however, may be gained in this area by examining the glycolytic enzyme activities in male and female skeletal muscle. For example, it is frequently observed that women have lower activities of hexokinase, phosphofructokinase and lactate dehydrogenase than men (Simoneau et al., 1985; Simoneau and Bouchard, 1989). While these results suggest that women rely less on anaerobic glycolysis as an energy source than men, more research is needed to determine the effects of these gender differences on the relative fatigability of males and females during exercise of short duration.

In an attempt to determine the mechanisms behind the gender difference in $\mathrm{CHO}$ metabolism, and the related fatigue resistance of females, many researchers have focused on the effects of estrogen. In a study by Kendrick and colleagues (1987), oophorectomized rats were treated with various doses of estradiol and exercised to exhaustion on a treadmill. Kendrick found that estradiol treated rats were able to run significantly longer before exhaustion, in a dose-dependent manner, than sham injected rats. The estradiol treated rats were also found to have significantly lower degrees of 
glycogen depletion in the skeletal muscle, myocardial and hepatic tissues than the sham injected rats. Rooney et al. in (1993), found similar glycogen sparing properties of the female sex hormone, as estradiol treated male rats did not show significant muscle glycogen depletion until 90 min. of treadmill running whereas oil injected rats were significantly depleted of muscle glycogen after $30 \mathrm{~min}$. Rooney also measured plasma FFA concentrations and muscle triacylglycerol content, and concluded that the significant glycogen sparing effects of estradiol are secondary to an estradiol-mediated increase in lipid substrate availability during exercise. While the studies by Kendrick and Rooney helped to elucidate the glycogen sparing, and therefore fatigue resistant properties of estradiol during exercise of long duration, much less is known about the possible fatigue resisting properties of estradiol during short duration exercise where glycogen depletion is not a factor in fatigue. Studies by Lewis (1997), and West (1995), however, have suggested that the female advantage in fatigue resistance cannot be completely explained by estradiol-mediated glycogen sparing, as they found the gender difference in fatigue to exist in exercise of very short duration. Similarly, Hicks and McCartney (1996), found post-menopausal women to be more fatigue resistant than age-matched men during 3 minutes of intermittent elbow flexor and ankle dorsiflexor exercise.

\subsection{Summary and Statement of Purpose}

It is generally accepted that the age-associated loss of strength and muscle mass results in a decreased ability to maintain any given absolute load as compared to the young. However, much less is known about how the complex muscular changes that 
accompany the aging process effect the relative fatigue resistance in the elderly. Those who have investigated this area with the use of voluntary fatigue protocols have often failed to account for possible age-related changes in motor unit activation (Aniansson et al. 1978; Larsson and Karlsson, 1978; Petrofsky and Lind, 1975), while those who used electrically-induced fatigue protocols have not accounted for the age-related leftward shift in the force frequency curve (Cupido et al., 1992; Davies et al., 1986; Klein et al., 1988). While no concrete conclusions have been drawn, it has been hypothesized that a histological shift toward Type I muscle fibre predominance in the elderly may enhance their fatigue resistance (Narici et al., 1991). Furthermore, some researchers have shown an age-related loss of muscle membrane excitability at rest (Vandervoort and McComas, 1986), while others have found the elderly to demonstrate the same maintenance of membrane excitability during fatiguing exercise as young adults (Hicks et al., 1992). Finally, it is also not fully understood how possible age-related changes in muscular blood flow (Coggan et al., 1992), and substrate metabolism (Fitts et al., 1984), may affect the fatigue resistance of elderly men and women.

While the gender difference in fatigability is well documented (Lewis et al., 1997; Maughan et al., 1986; West et al., 1995), it is still unclear what mechanisms underlie the female advantage in muscular endurance. Some have shown males to possess a greater proportion of Type II muscle fibres than females (Miller et al., 1993; Simoneau et al., 1985), while others have shown no such gender difference in fibre type distribution (Sale et al., 1987; Schantz et al., 1983). Further, while estrogen has been shown to possess fatigue resisting qualities in rats during exercise of long duration (Kendrick et al., 1987), 
much less is known about its influence on short duration exercise in humans. Although Hicks and McCartney (1996) found post-menopausal women to be significantly more fatigue resistant than age-matched males during 3 minutes of intermittent MVC's of the elbow flexors and ankle dorsiflexors, they did not test young adults, and it is possible that they would have found a greater (estrogen-related) gender difference in fatigue among young males and females.

It has also been theorized that the greater absolute strength in males may lead to a greater local muscle ischemia during exercise as compared to females, and hence, result in a lower fatigue resistance. Although Lewis et al. (1997) found evidence to contradict this theory, no electromyographical measures were made and motor unit activation was not considered.

Finally, de Haan and colleagues (1988), theorized that the greater fatigability of males may be due to a gender difference in muscle economy, which may result from a greater number of sarcomeres in series in the muscle fibres of men as compared to women. This theory, however, has yet to be adequately addressed.

The purpose of the present study was therefore, to examine the relationships between age, gender and fatigue resistance, with consideration to the relative influence that estrogen status, membrane excitability, absolute force and fibre length have over the development of fatigue during short duration exercise of the adductor pollicis muscle. 


\section{$\underline{\text { References }}$}

Aniansson, A., Grimby, G., Hedberg, M., Rundgren, A. and Sperling, L. (1978). Muscle function in old age. Scand. J. Rehab. Med. (Suppl.) 6: 43-49.

Belanger, A.Y. and McComas, A.J. (1981). Extent of motor unit activation during effort. J. Appl. Physiol. 51(5): 1131-1135.

Bell, D.G. and Jacobs, I. (1990). Muscle fibre area, fibre type and capillarization in male and female body builders. Can. J. Spt. Sci. 15(2): 115-119.

Bellemare, F. and Garzaniti, N. (1988). Failure of neuromuscular propagation during human maximal voluntary contraction. J. Appl. Physiol. 64(3): 1084-1093.

Bigland-Ritchie, B., Jones, D.A. and Woods, J.J. (1979). Excitation frequency and muscle fatigue: Electrical responses during human voluntary and stimulated contractions. Exp. Neurol. 64: 414-427.

Bigland-Ritchie, B., Kukulka, C.G., Lippold, O.C.J. and Woods, J.J. (1982). The absence of neuromuscular transmission failure in sustained maximal voluntary contractions. J. Physiol. 330: 265-278.

Bigland-Ritchie, B., Johansson, R., Lippold, O.C.J. and Woods, J.J. (1983). Contractile speed and EMG changes during fatigue of sustained maximal voluntary contractions. J. Neurophysiol. 50: 313-324.

Bigland-Ritchie, B., Furbush, F. and Woods, J.J. (1986). Fatigue of intermittent submaximal voluntary contractions: central and peripheral factors. J. Appl. Physiol. 61(2): 421-429.

Brookes, S.V. and Faulkner, J.A. (1988). Contractile properties of skeletal muscles from young, adult and aged mice. J. Physiol. 472: 61-80.

Brookes, S.V. and Faulkner, J.A. (1994). Isometric shortening and lengthening contractions of muscle fibre segments from adult and old mice. Am. J. Physiol. 267: C507-C513.

Brown, W.F. (1972). A method for estimating the number of motor units in thenar muscles and the changes in motor unit count with ageing. J. Neurol. Neurosurg. Psychiat. 35: 845-852.

Bruce, S.A., Newton, D. and Woledge, R.C. (1989). Effect of age on voluntary force and cross-sectional area of human adductor pollicis muscle. Q. J. Exp. Physiol. 74, 359-362. 
Burke, W.E., Tuttle, W.W., Thompson, C.W., Janney, C.D. and Weber, R.J. (1953). The relation of grip strength and grip strength endurance to age. J. Appl. Physiol. 5: 628-630.

Campbell, M.J., McComas, A.J. and Petito, F. (1973). Physiological changes in aging muscles. J. Neurol. Neurosurg. Psychiatry 36:174-182.

Cartee, G.D. and Farrar, R.P. (1988). Exercise training induces glycogen sparing during exercise by old rats. J. Appl. Physiol. 63: 259-265.

Cartee, G.D. (1994). Influence of age on skeletal muscle glucose transport and glycogen metabolism. Med. Sci. Sports Exerc. 26(5): 577-585.

Coggan, A.R., Spina, R.J., King, D.S., Rogers, M.A., Brown, M., Nemeth., P.M. and Holloszy, J.O. (1992). Histochemical and enzymatic comparison of the gastrocnemius muscle of young and elderly men and women. J. Gerontol. 47(3): B71-76.

Collumbine, H., Bibile, S.W., Wikramanayake, T.W. and Watson, R.S. (1950). Influence of age, sex, physique and muscular development on physical fitness. J. Appl.

Physiol. 2: 488-511.

Costill, D.L., Fink, W.J., Getchell, L.H., Ivy, J.L., Witzman, F.A. (1979). Lipid metabolism in skeletal muscle of endurance-trained males and females. J. Appl. Physiol. 47: 787-791.

Cupido, C.M., Hicks, A.L. and Martin, J. (1992). Neuromuscular fatigue during repetitive stimulation in elderly and young adults. Eur. J. Appl. Physiol. 65: 567-572.

Davies, C.T.M., White, M.J. and Young, K. (1983). Electrically evoked and voluntary maximal isometric tension in relation to dynamic muscle performance in elderly male subjects, aged 69 years. Eur. J. Appl. Physiol. 51: 37-43.

Davies, C.T.M., Thomas, D.O. and White, M.J. (1986). Mechanical properties of young and elderly human muscle. Acta Med. Scand. (Suppl.) 711: 219-226.

de Haan, A., Rexwinkel, R., van Doorn, J., Westra, H., Hollander, A., Huijing, P., Woittiez, R. and Sargeant, A. (1988). Influence of muscle dimensions on economy of isometric exercise in rat medial gastrocnemius muscles in situ. Eur. J. Appl. Physiol. 57: 64-69.

De Luca, A., Mambrini, M. and Camerino, D.C. (1990). Changes in membrane ionic conductances and excitability characteristics of rat skeletal muscle during aging. Pflug. Arch. 415: 642-644. 
Denis, C., Chatard, J.C., Dormois, D., Linossier, M.T., Geyssant, A. and Lacour, J.P. (1986). Effects of endurance training on capillary supply of human skeletal muscle on two age groups (20 and 60 years). J. Physiol. 81: 379-383.

Doherty, T.J., Vandervoort, A.A., Taylor, A.W. and Brown, W.F. (1993). Effects of motor unit losses on strength in older men and women. J. Appl. Physiol. 74(2): 868-874.

Dudley, G.A. and Fleck, S.J. (1984). Metabolite changes in aged muscle during stimulation. J. Gerontol. 39(2): 183-186.

Eberstein, A. and Sandow, A. (1963). Fatigue mechanisms in muscle fibres. In: The effect of use and disuse of neuromuscular function. Gutmann, E. and Hnik, P. (eds.). Prague: Czech. Acad. Sci. 515-526.

Eddinger, T.J., Cassens, R.G. and Moss, R.L. (1986). Mechanical and histochemical characterization of skeletal muscles from senescent rats. Am. J. Physiol. 251: C421-430.

Ermini, M. and Verzar, F. (1968). Decreased restitution of creatine phosphate in white and red skeletal muscles during aging. Experientia. 24: 902-904.

Essen-Gustavsson, B. and Borges, O. (1986). Histochemical and metabolic characteristics of human skeletal muscle in relation to age. Acta. Physiol. Scand. 126: 107-114.

Fitts, R.H., Troup, J.P., Witzman, F.A. and Holloszy, J.O. (1984). The effect of aging and exercise on skeletal muscle function. Mech. Age. Develop. 27: 161-172.

Fitts, R.H. (1994). Cellular mechanisms of muscle fatigue. Physiological Reviews. 74(1): 49-94.

Frolkis, V.V., Martynenko, O.A. and Zamostyan, V.P. (1976). Aging of the neuromuscular apparatus. Gerontol. 22: 244-279.

Galea, V. (1996). Changes in motor unit estimates with aging. J. Clin. Neurophysiol. 13(3): 253-260.

Gandevia, S.C., Herbert, R.D. and Leeper, J.B. (1998). Voluntary activation of the human elbow flexor muscles during maximal concentric contractions. J. Physiol. 512 (Pt 2): 595-602.

Garland, J.S., Garner, S.H. and McComas, A.J. (1988). Reduced voluntary electromyographic activity after fatiguing stimulation of human muscle. J. Physiol. 401: 547-556. 
Govindasamy, D. and Paterson, D. (1994). The physiology of aging. In: Zeigler, E., editor. Physical activity and the older adult: A knowledge base for managing exercise programs. Illinois: Stipes Publishing Company, 2-5.

Grimby, L., Hannerz, J. and Hedman, B. (1981). The fatigue and voluntary discharge properties of single motor units in man. J. Physiol. 316: 545-554.

Grindrod, S., Round, J.M. and Rutherford, O.M. (1987). Type 2 fibre composition and force per cross-sectional area in the human quadriceps (Abstract). J. Physiol. 390: $154 \mathrm{P}$.

Haidet, G.C. and Parsons, D. (1991). Reduced exercise capacity in senescent beagles: an evaluation of the periphery. Am J. Physiol. 260: H173-182.

Hakkinen, K. and Hakkinen, A. (1991). Muscle cross-sectional area, force production and relaxation characteristics in women at different ages. Eur. J. Appl. Physiol. 62: 410-414.

Hicks, A.L. and McComas, A.J. (1989). Increased sodium pump activity following stimulation of rat soleus muscles. J. Physiol. 414: 337-349.

Hicks, A.L., Cupido, C.M., Martin, J. and Dent, J. (1992). Muscle excitation in elderly adults: The effects of training. Muscle Nerve 15: 87-93.

Hicks, A. and McCartney, N. (1996). Gender Difference in isometric contractile properties and fatigability in elderly human muscle. Can. J. Appl. Phys. 21(6): 441-454.

Hodgkin, A.L. and Katz, B. (1949). The effect of sodium ions on the electrical activity of the giant axon of the squid. J. Physiol. 108: 37-77.

Holloszy, J.O., Chen, M., Cartee, G.D. and Young, J.C. (1991). Skeletal muscle atrophy in old rats: Differential changes in the three fibre types. Mech. Ageing Dev. 60: 199-213.

Howell, J.N. and Snowdowne, K.W. (1981). Inhibition of tetanus tension by elevated extracellular calcium concentration. Am. J. Physiol. 240: C193-C200.

Irion, G.L., Vasthare, U.S. and Tuma, R.F. (1987). Age-related change in skeletal muscle blood flow in the rat. J. Gerontol. 42(6): 660-665.

Jacobs, I., Bar-Or, O., Karlsson, J., Dotan, R., Tesch, P., Kaiser, P. and Inbar, O. (1982). Changes in muscle metabolites in females with 30-s exhaustive exercise. Med. Sci. Sports Exerc. 14(6): 457-460. 
Jakobsson, F., Borg, K. and Edstrom, L. (1990). Fibre-type composition, structure and cytoskeletal protein location of fibres in anterior tibialis muscle. Comparison between young adults and physically active aged humans. Acta Neuropathol. 80: 459-468.

Jones, D.A., Bigland-Ritchie, B. and Edwards, R.H.T. (1979). Excitation frequency and muscle fatigue: Mechanical responses during voluntary and stimulated contractions. Exp. Neurol. 64: 401-413.

Jones, D.A. (1981). Muscle fatigue due to changes beyond the neuromuscular junction. In: Porter, R. and Whelan, J. (eds.) Human muscle fatigue: Physiological mechanisms. Pitman Medical, London, 178-196.

Kanehisa, H., Ikegawa, S. and Fukunaga, T. (1994). Comparison of muscle crosssectional area and strength between untrained men and women. Eur. J. Appl. Physiol. 68(2): 148-154.

Kendrick, Z.V., Steffen, C.A., Rumsey, W.L. and Goldberg, D.I. (1987). Effect of estradiol on tissue glycogen metabolism in exercised oophorectomized rats. J. Appl. Physiol. 63(2): 492-496.

Kjeldson, K. (1987). Regulation of the concentration of ${ }^{3} \mathrm{H}$-ouabain binding sites in mammalian skeletal muscle - Effects of age, K-depletion, thyroid status and hypertension. Dan. Med. Bull. 34: 15-46.

Klein, C., Cunningham, D.A., Paterson, D.H. and Taylor, A.W. (1988). Fatigue and recovery contractile properties of young and elderly men. Eur. J. Appl. Physiol. 57: 684-690.

Larsson, L. and Karlsson, J. (1978). Isometric and dynamic endurance as a function of age and skeletal muscle characteristics. Acta. Physiol. Scand. 104: 129-136.

Larsson, L. and Salviati, G. (1989). Effects of age on calcium transport activity of sarcoplasmic reticulum in fast-and slow-twitch rat muscle fibres. J. Physiol. 419: 253-264.

Laubach, L. (1976). Comparative muscular strength of men and women: A review of the literature. Aviat. Space Environ. Med. 47(5): 534-542.

Lewis, S.F., Rock, P.B., Muza, S.R. Lammi, E., Moore, L.G., Cymerman, A. and Fulco, C.S. (1997). Slower rate of adductor pollicis muscle fatigue in women than in men. (Abstract). Med. Sci. Sport Exer. Suppl. 29(5): S92. 
Lexell, J., Taylor, C. and Sjostrom, M. (1988). What is the cause of aging atrophy? Total number, size and proportions of different fibre types studied in whole vastus lateralis muscle from 15 to 83 year old men. J. Neurol. Sci. 84: 275-294.

Loscher, W.N., Cresswell, A.G. and Thorstensson, A. (1996a). Central fatigue during long lasting submaximal contraction of the triceps surae. Exp. Brain Res. 108(2): 305-314.

Loscher, W.N., Cresswell, A.G. and Thorstensson, A. (1996b). Excitatory drive to the alpha-motoneuron pool during a fatiguing submaximal contraction in man. $\mathbf{J}$. Physiol. 491 (Pt 1): 271-280.

Li, Z., Krause, D.N., Doolen, S. and Duckles, S.P. (1997). Ovariectomy eliminates sex differences in rat tail artery response to adrenergic nerve stimulation. Am. J. Physiol. 272(4 pt 2): H1819-H1825.

Marsden, C.D., Meadows, J.C. and Merton, P.A. (1971). Isolated single motor units in human muscle and their rate of discharge during maximum voluntary effort. $\mathbf{J}$. Physiol. 217: 12P-13P.

Maughan, R.J., Watson, J.S. and Weir, J. (1983). Strength and cross-sectional area of human skeletal muscle. J. Physiol. 338: 37-49.

Maughan, R.J., Nimmo, M.A. and Harmon, M. (1985). The relationship between muscle myosin ATP-ase activity and isometric endurance in untrained male subjects. Eur. J. Appl. Physiol. 54(3): 291-296.

Maughan, R.J., Harmon, M., Leiper, J.B., Sale, D. and Delman, A. (1986). Endurance capacity of untrained males and females in isometric and dynamic muscular contractions. Eur. J. Appl. Physiol. 55: 395-400.

Merton, P.A. (1954). Voluntary strength and fatigue. J. Physiol. 123: 553-564.

Miller, A.E.J., MacDougall, J.D. Tarnopolsky, M.A. and Sale, D.G. (1993). Gender differences in strength and muscle fibre characteristics. Eur. J. Appl. Physiol. 66: 254-262.

Milner-Brown, H.S. and Miller, R.G. (1986). Muscle membrane excitation and impulse propogation velocity are reduced during muscle fatigue. Muscle Nerve 9: 367374.

Narici, M.V., Bordini, M. and Cerretelli, P. (1991). Effect of aging on human adductor pollicis muscle function. J. Appl. Physiol. 71(4): 1277-1281. 
Nygaard, E. (1981). Skeletal muscle fibre characteristics in young women. Acta Physiol. Scand. 112: 299-304.

Petrofsky, J.S. and Lind, A.R. (1975). Aging, isometric strength and endurance, and cardiovascular response to static effort. J. Appl. Physiol. 38(1): 91-95.

Ruby, B.C. (1999). Gender differences in carbohydrate metabolism: Rest, exercise and post-exercise. In: Tarnopolsky, M.A., editor. Gender differences in metabolism: Practical and nutritional implications. Boca Raton, Florida: CRC Press LLC, 6185.

Rooney, T.P., Kendrick, Z.V., Carlson, J., Ellis, G.S., Matakevich, B., Lorusso, S.M. and McCall, J.A. (1993). Effect of estradiol on the temporal pattern of exerciseinduced tissue glycogen depletion in male rats. J. Appl. Physiol. 75(4): 15021506.

Sale, D.G., MacDougall, J.D., Alway, S.E. and Sutton, J.R. (1987). Voluntary strength and muscle characteristics in untrained men and women and male bodybuilders.

J. Appl. Physiol. 62(5): 1786-1793.

Sale, D.G. (1999). Neuromuscular Function. In: Tarnopolsky, M.A., editor. Gender differences in metabolism: Practical and nutritional implications. Boca Raton, Florida: CRC Press LLC, 61-85.

Schantz, P., Randell-Fox, E., Hutchinson, W., Tyden, A. and Astrand, P.O. (1983). Muscle fibre type distribution, muscle cross-sectional area and maximal voluntary strength in humans. Acta Physiol. Scand. 117: 219-226.

Sieck, G.C. and Prakash, Y.S. (1995). Fatigue at the neuromuscular junction: Branch point vs. presynaptic vs. postsynaptic mechanisms. Adv. Exp. Med. Biol. 384: 83-100.

Simoneau, J.A., Lortie, G., Boulay, M.R., Thibault, M.C., Theriault, G. and Bouchard, C. (1985). Skeletal muscle histochemical and biochemical characteristics in sedentary male and female subjects. Can. J. Physiol. Pharmacol. 63: 30-35.

Simoneau, J.A. and Bouchard, C. (1989). Human variation in skeletal muscle fibre type proportion and enzyme activities. Am. J. Physiol. 257: E567-E572.

Smith, D.O. and Weiler, M.H. (1987). Acetylcholine metabolism and choline availability at the neuromuscular junction of mature adult and aged rats. J. Physiol. 383: 693709 . 
Stalberg, R. and Fawcett, P.R.W. (1982). Macro EMG in healthy subjects of different ages. J. Neurol. Neurosurg. Psychiat. 45: 870-878.

Tarnopolsky, L.J., MacDougall, J.D., Atkinson, S.A., Tarnopolsky, M.A. and Sutton, R.J. (1990). Gender difference in substrate for endurance exercise. J. Appl. Physiol. 68: 302-308.

Tarnopolsky, M.A., Atkinson, S.A., Phillips, S.M. and MacDougall, J.D. (1995). Carbohydrate loading and metabolism during exercise in men and women. J. Appl. Physiol. 78: 1360-1368.

Tarnopolsky, M.A. (1999). Gender differences in metabolism: Practical and nutritional implications. Boca Raton, Florida: CRC Press LLC, 61-85.

Taylor, D.J., Crowe, M., Bore, P.J., Styles, P., Arnold, D.L. and Radda, G.K. (1984). Examination of the energetics of aging skeletal muscle using nuclear magnetic resonance. Gerontol. 30: 2-7.

Tesch, P., Sjodin, B. and Karlsson, J. (1978). Muscle fatigue and its relation to lactate accumulation and LDH activity in man. Acta. Physiol. Scand. 103: 413-420.

Tomlinson, B.E. and Irving, D. (1977). The numbers of limb motor neurons in human lumbrosacral cord throughout life. J. Neurol. Sci. 34: 213-219.

Tucek, S. and Gutmann, E. (1973). Choline acetyltransferase activity in muscles of old rats. Exp. Neurol. 38: 349-360.

Vandervoort, A.A. and McComas, A.J. (1986). Contractile changes in opposing muscles of the human ankle joint with aging. J. Appl. Physiol. 61: 361-367.

West, W., Hicks, A., Clements, L. and Dowling, J. (1995). The relationship between voluntary electromyogram, endurance time and intensity of effort in isometric handgrip exercise. Eur. J. Appl. Physiol. 71: 301-305.

Winegard, K., Hicks, A. and Vandervoort, A. (1997). An evaluation of the length-tension relationship in elderly human plantarflexor muscles. J. Gerontol. 52(6): B337B343.

Young, A. (1984). The relative isometric strength of type I and type II muscle fibres in the human quadriceps. Clin. Physiol. 4: 23-32.

Young, A., Stokes, M. and Crowe, M. (1985). The size and strength of the quadriceps muscles of old and young men. Clin. Physiol. 5(2): 145-154. 


\section{Chapter II}

\section{The Effect of Age and Gender on the Relative Fatigability of the Human Adductor Pollicis Muscle}

\subsection{Introduction}

Age-related strength losses are well documented in both men and women, and have been found to occur in large muscle groups such as the leg extensors (Hakkinen and Hakkinen, 1991) and elbow flexors (Hicks and McCartney, 1996), as well as in the smaller intrinsic hand muscles such as the adductor pollicis (Bruce et al., 1989; Narici et al., 1991). This loss of strength in the elderly, which accelerates after the age of approximately 60 years (Govindasamy and Paterson, 1994), has been attributed in large part to the loss of Type I and Type II muscle fibres, and a selective atrophy of Type II muscle fibres (Lexell et al., 1988).

The age-related loss of muscle mass and strength dictate that at any absolute load the elderly should fatigue more quickly than the young. Some of the earlier work examining aging and fatigue supports this contention (Burke et al., 1953; Collumbine et al., 1950), and while such findings have practical implications, it may be of greater interest to investigate the age-related changes in relative fatigability. Despite the extensive amount of research in this area, there is still no clear consensus as to the relative fatigue resistance in elderly compared to young adults. 
For example, while some researchers have found either a similar (Klein et al., 1988; Larsson and Karlsson, 1978; Petrofsky and Lind, 1975) or enhanced (Aniansson et al., 1978; Narici et al., 1991) resistance to fatigue in the elderly as compared to the young, others have found age-related deficits in muscular endurance (Cupido et al., 1992; Davies et al., 1983; Davies et al., 1986). The discrepancy of these findings may be related to differences in the fatigue paradigms used, in addition to any number of confounding factors that accompany the aging process, including the general decline in physical activity (Govindasamy and Paterson, 1994), as well as subtle changes in the response of elderly muscle to electrical stimulation. With respect to the latter, it has been shown that, with aging, comes a leftward shift in the force-frequency curve (Narici et al., 1991). Therefore, at any given stimulation frequency up to approximately $50 \mathrm{~Hz}$, the elderly will produce contractions at a higher percentage of their maximal tetanic force, as compared to the young, and thus, their fatigue resistance may be underestimated. It should be noted that many of those who found age-related deficits in muscular endurance, employed electrically-induced fatigue protocols in which the young and elderly were stimulated at the same frequency (Cupido et al., 1992; Davies et al., 1983; Davies et al., 1986). Similarly, studies which utilize voluntary fatigue protocols frequently fail to monitor the percent motor unit activation, and therefore, may produce results that are confounded by possible age-related changes in central drive (Aniansson et al., 1978; Larsson and Karlsson, 1978).

Gender differences in muscle fatigue are well documented, and it is generally agreed that females exhibit a greater relative fatigue resistance as compared to males 
(Lewis et al., 1997; Maughan et al., 1986; West et al., 1995). This female advantage has been shown to exist in a variety of muscles, and seems to persist despite the aging process (Hicks and McCartney, 1996). The mechanisms responsible for this gender difference, however, are not completely resolved.

It has been suggested that the male strength advantage (for a review see Sale, 1999), may be partially responsible for the gender difference in fatigue, as the lower absolute force production in females during relative endurance tasks, may translate into less local muscle ischemia and therefore a greater fatigue resistance compared to males. Lewis et al. (1997), tested this hypothesis with a unique protocol that matched men and women for adductor pollicis strength. The results of this study showed that despite equal initial strength, women remained decisively less fatigable than men during intermittent isometric contractions. Lewis et al., however, did not measure the electrical activity of the muscle or the percent motor unit activation, and therefore, his results may have been confounded by differences in central drive between genders.

de Haan et al. (1988), theorized that differences in muscle length between males and females, may contribute to the gender difference in fatigue. Specifically, de Haan et al. stated that the number of sarcomeres in series in a muscle fibre acts to increase the energy utilization during contraction, but does not enhance force production, the latter being a function of muscle cross-sectional area. Therefore, when comparing two muscles of equal cross-sectional area, the longer of the two will require more energy for any given force production. Since males, in general, are larger than females, and thus have longer muscles, de Haan's reasoning may be relevant in the discussion of gender differences in 
fatigue. To expand, the fewer number of sarcomeres in series in female muscle may allow for a more economical force production, which in turn, would translate into a greater fatigue resistance in women. The de Haan theory, however, has yet to be adequately tested.

Gender differences in substrate metabolism may also bear great influence over the gender differences in fatigue resistance. In a series of rigorously controlled experiments by Tarnopolsky et al. $(1990 ; 1995)$, it was determined that females have a significantly lower reliance on carbohydrate $(\mathrm{CHO})$ metabolism during submaximal aerobic exercise as compared to males. Although gender differences in fibre type distribution remain controversial (Schantz et al., 1983; Simoneau and Bouchard, 1989), greater TypeI/II fibre area ratios in females may account for some of the gender difference in $\mathrm{CHO}$ metabolism. A more likely explanation for this reduced $\mathrm{CHO}$ dependence in females, is provided by the frequently observed glycogen sparing properties of estrogen (Kendrick et al., 1987; Rooney et al., 1993). However, while the fatigue resistant properties of estrogen have been frequently demonstrated during exercise of long duration, much less is known about the possible fatigue resisting properties of estrogen during short duration exercise, where glycogen depletion does not factor in fatigue. Although Hicks and McCartney (1996) found post-menopausal women to be more fatigue resistant than agematched men during 3 minutes of intermittent MVC's of the elbow flexors and dorsiflexors, they did not include young adults in their investigation, and it is possible that they would have found a significantly greater (estrogen-related) gender difference in fatigue among young males and females. 
The purpose of the present study was therefore, to examine the relationships between age, gender and fatigue resistance, with consideration to the relative influence that estrogen status, membrane excitability, absolute force and fibre length have over the development of fatigue during short duration exercise of the adductor pollicis muscle.

\subsection{Methods}

\subsection{1 $\underline{\text { Subjects }}$}

A total of 48 subjects, classified by age and gender, participated in this study. Twelve subjects were represented in each of the following groups: young males ( $25.3 \pm$ $2.1 \mathrm{yrs})$, young females ( $23.5 \pm 2.1 \mathrm{yrs})$, elderly males ( $71.7 \pm 5.6 \mathrm{yrs})$ and elderly females ( $69.5 \pm 4.6 \mathrm{yrs})$. The young subjects were recruited from McMaster University, and all were self-described as active, although none were considered elite athletes. Each of the young females reported as being eumenorrheic and none were taking oral contraceptives at the time of testing. Further each young female was tested in the late follicular phase of the menstral cycle (10-13 days after the onset of menstruation). The elderly subjects were recruited from the "Mac Seniors" exercise program, and thus, all were engaged in active lifestyles consisting of at least, twice weekly, supervised exercise training. None of the elderly subjects were taking $\beta$-blocker medications, and each of the elderly females reported as being post-menopausal and not on hormone replacement therapy. Further, all 48 subjects had abstained from caffeine consumption for at least 12 hours prior to testing, and none of the subjects had any contraindication to electrical 
muscle stimulation or lacked the full range of motion of the thumb. Informed consent was obtained from each subject (Appendix A), and the study was approved by the McMaster University Ethics Committee.

\subsubsection{Stimulating and recording}

Each subject placed his/her non-dominant hand palm-down in a custom designed apparatus. The hand was tightly secured in a fixed position, such that the angle between the thumb and index finger was $85^{\circ}$. This degree of thumb abduction had been previously determined as the optimal angle for adductor pollicis force production (Appendix B). An adjustable chair faced the apparatus such that each subject could sit comfortably with his/her pronated forearm perpendicular to the plane of the body with the elbow flexed at approximately $135^{\circ}$. Just prior to skin preparation, each subject had his/her hand wrapped in a heating pad for approximately 5 minutes so that the skin temperature measured at the palm was between $30-35^{\circ} \mathrm{C}$.

After cleansing and lightly abrading the skin on both sides of the hand and wrist, a stimulating electrode (coated with conducting cream) was placed on the volar surface of the forearm, just proximal to the wrist and medial to the midline, with the cathode distal. In this position the electrode would lie directly over the ulnar nerve. A Medi-trace pellet disposable electrode (Graphic Controls Canada Ltd., patent no. 1144606), consisting of a silver-silver chloride disc measuring $1 \mathrm{~cm}$ in diameter, was used as the recording electrode for EMG activity, and was secured over the belly of the adductor pollicis muscle. A $25 \mathrm{~mm} \times 5 \mathrm{~mm}$ silver strip was used as the reference electrode and was taped 
to the distal phalanx of the middle finger. Another Medi-trace disposable electrode was secured to the back of the wrist, between the recording and stimulating electrodes, to serve as a ground. A splint was put on the palmar side of the thumb in order to minimize flexion at the distal joint, and subjects then placed their thumb into a steel ring such that the ring straddled the distal joint. The steel ring was fixed to a strain gauge and the strain gauge mount was magnetized to the table surface and could be moved to attain any desired thumb angle (Fig. 1).

\subsubsection{Baseline measures}

\subsubsection{Evoked twitches}

To avoid any post-tetanic potentiation (Houston et al., 1985; Vandervoort et al., 1983), baseline twitches were performed prior to the baseline maximal voluntary contractions (MVC).

A stimulator delivered rectangular voltage pulses of $100 \mu$ s to evoke single twitches. The voltage was adjusted until there was no further increase in force, and this voltage was then used for all subsequent stimulations. Two twitches and corresponding compound muscle action potentials (M-waves) were then recorded and the greater of the two was determined to be the peak twitch torque. Data were streamed continuously to a computer disk and displayed in real time using a Dataq waveform scrolling board (WFS- 
Figure 1.

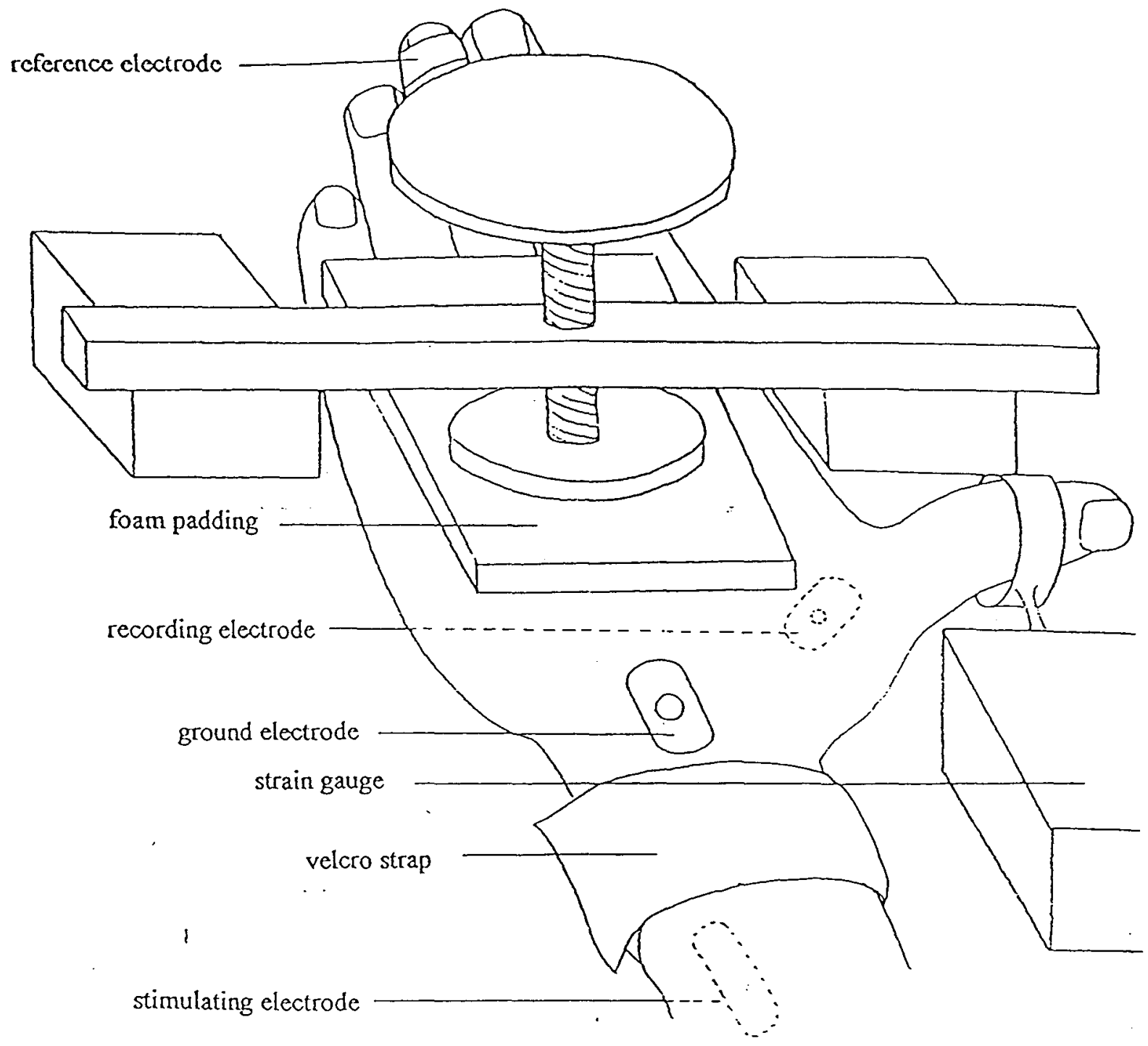


200PC; Dataq Instruments, Akron, Ohio). Measures were made for peak twitch torque (PT), time to peak twitch torque (TPT), half-relaxation time (HRT), maximum rate of torque development (MRTD), as well as $\mathrm{M}$-wave amplitude $\left(\mathrm{M}_{\mathrm{amp}}\right)$, duration $\left(\mathrm{M}_{\mathrm{dur}}\right)$ and area $\left(\mathrm{M}_{\text {area }}\right)$ using the Windaq and custom designed software (Dataq Instruments).

\subsubsection{Maximum voluntary contractions}

Following all measurements of the evoked responses, subjects performed two maximal voluntary contractions, and the greater of the two was determined to be the baseline MVC. Verbal encouragement from the experimenter as well as visual feedback from the force monitoring screen were both provided in an attempt to fully motivate each subject to achieve their maximum voluntary force. A single twitch was interpolated on each 5s MVC to estimate the amount of motor unit activation, according to the method of Belanger and McComas (1981). A 0.5s window of the electromyogram (EMG) associated with the MVC for each joint angle was analyzed for determination of voluntary EMG; average EMG (AEMG) was then determined by dividing the voluntary EMG by the $0.5 \mathrm{~s}$ time frame.

\subsubsection{Fatigue Protocol}

The fatigue protocol consisted of intermittent isometric MVC's of the adductor pollicis. Subjects repeatedly alternated $5 \mathrm{~s}$ maximal voluntary contractions with $2 \mathrm{~s}$ of rest, for a total duration of $3 \mathrm{~min}$. In each $2 \mathrm{~s}$ rest period a single twitch of the adductor pollicis was evoked, and single twitches were interpolated on 10 of the MVC's (approximately 
every third MVC), in order to determine the degree of motor unit activation throughout the fatigue protocol. Although $25 \mathrm{MVC}$ 's and twitches were performed during the fatigue protocol, only 10 of each were recorded and analyzed, which corresponded to approximately every third contraction. Subjects were instructed to focus their attention on a red light that signaled each of the MVC's, while the experimenter provided constant verbal encouragement. The importance of producing tension strictly in the plane of adduction (with as little force as possible being produced in the direction of flexion or opposition) was emphasized to each subject before the baseline measures, and again just prior to the fatigue protocol. Immediately following the final MVC, subjects were reminded to stay as still as possible throughout the subsequent recovery phase.

The fatigue index of both the voluntary and evoked force was also calculated using the following formula:

$$
\mathrm{FI}(\%)=\frac{\text { Force }_{\text {initial }}-\text { Force }_{\text {final }}}{\text { Force }_{\text {initial }}} \times 100
$$

\subsubsection{Recovery}

The recovery phase was 10 minutes in duration, during which time, 7 single twitches were evoked in each subject. The 7 twitches occurred at 0.5 min., 1.0 min., 1.5 $\min ., 2.0 \mathrm{~min} ., 3.0 \mathrm{~min} ., 5.0 \mathrm{~min}$. and $10.0 \mathrm{~min}$. into the recovery period. Shortly after the final evoked twitch, subjects performed one last 5 second MVC in order to determine the degree of voluntary force recovery. 


\subsubsection{EMG processing}

All EMG signals, corresponding to the M-wave and voluntary EMG data, were amplified using a Honeywell accudata EMG bio-amplifier at a sampling rate of $2.5 \mathrm{kHz}$ and a bandwidth of $15 \mathrm{~Hz}$ to $1.5 \mathrm{kHz}$. The Windac software program, ACODAS, (Dataq Instruments, Dayton, Ohio) was used to analyze the characteristics of the M-wave, and to full-wave digitally rectify and calculate the area under the curve for the voluntary EMG signals.

The AEMG:M-wave ratio was also calculated (AEMG $(\mathrm{mV}) / \mathrm{M}$-wave area $\mu \mathrm{V} \cdot \mathrm{s})$. This value relates the evoked muscle membrane excitability to the voluntary activation of the muscle and was used to help determine potential mechanisms for any changes in muscle activation.

\subsubsection{Measurement of Hand Dimensions}

Following the recovery period, two measurements of hand dimensions were made for each subject. The first measure was that of the proximal phalanx of the thumb, which was defined as the distance between the mid-points of its proximal and distal joints. This measurement, which represents the moment arm of torque production, was made on the dorsal side of the thumb. Dividing the measured torque by this moment arm length, allowed for a closer approximation of the relative muscle forces between subjects, i.e. without the confounding factor of variable moment arm lengths.

The second measurement estimated the length of the adductor pollicis muscle from origin to insertion. The distance between the third metacarpal and the base of the 
proximal phalanx of the thumb was used for this approximation (Salmons, 1995). This measure was made on the palmar side of the hand with the thumb abducted to $85^{\circ}$.

\subsubsection{Statistical analysis}

Each of the baseline dependent variables were analyzed with a two factor (age $\mathrm{x}$ gender) between subjects analysis of variance (ANOVA). The mechanical and electrical variables measured during the fatigue and recovery periods were analyzed with a three factor (age $\mathrm{x}$ gender $\mathrm{x}$ time) between subjects analysis of variance, with repeated measures for time. The Tukey A honestly significant difference post hoc test was used as needed to compare means. Multiple regression analysis was used to determine the relative contributions of age, gender, initial strength and adductor pollicis length to the development of fatigue. Statistical significance was established at $\mathrm{p} \leq 0.05$ and all data are presented as mean \pm standard deviation.

\section{$2.3 \quad$ Results}

\subsubsection{Baseline Measures}

\subsubsection{Peak Twitch Torque}

There were main effects found for both age and gender on evoked twitch torque. The young were found to produce significantly greater torques than the elderly $(0.85 \pm$ $0.22 \mathrm{Nm}$ vs. $0.60 \pm 0.22 \mathrm{Nm}$, respectively) and men were found to produce significantly 
greater torques than women $(0.86 \pm 0.26 \mathrm{Nm}$ vs. $0.59 \pm 0.15 \mathrm{Nm}$, respectively). These gender and age differences persisted after controlling for the different moment arm (proximal phalanx of the thumb) lengths between subjects (Table 1a). There was no significant age by gender interaction.

\subsubsection{Maximum Rate of Evoked Torque Development}

There were main effects for both age and gender on the MRTD. The peak rate of torque development for the young was significantly higher than that of the elderly $(22.3 \pm$ $5.6 \mathrm{Nm} / \mathrm{s}$ vs. $16.5 \pm 6.2 \mathrm{Nm} / \mathrm{s}$, respectively) and the peak rate of torque development for males was significantly higher than that of females $(23.1 \pm 6.5 \mathrm{Nm} / \mathrm{s}$ vs. $15.7 \pm 4.1 \mathrm{Nm} / \mathrm{s}$, respectively) (Table 1a). There was no significant age by gender interaction.

\subsubsection{Time to Peak Torque}

There were no main effects found for either age or gender on TPT and there was no age by gender interaction (Table 1a).

\subsubsection{Half-Relaxation Time}

There was a main effect for gender on HRT such that males had significantly shorter relaxation times than females $(68.9 \pm 9.9 \mathrm{~ms}$ vs. $78.8 \pm 16.9 \mathrm{~ms}$, respectively). There was no main effect for age and there was no age by gender interaction (Table 1a). 


\subsubsection{M-wave Characteristics}

There was a main effect for age on the M-wave area measures. The young produced significantly greater M-wave areas than the elderly $(0.082 \pm 0.014 \mathrm{mVs}$ vs. $0.056 \pm 0.017 \mathrm{mVs}$, respectively). There was no effect for gender on the M-wave area and there was no age by gender interaction (Table 1a).

There was also a main effect for age on the M-wave amplitude. The M-wave amplitudes produced by the young were significantly larger than those produced by the elderly $(19.8 \pm 2.8 \mathrm{mV}$ vs. $12.7 \pm 3.8 \mathrm{mV}$, respectively). There was no effect for gender on the M-wave amplitude and there was no age by gender interaction (Table 1a).

There was no significant effect for age or gender on the duration of the baseline M-wave. There was also no significant age by gender interaction (Table 1a).

Table 1a. Baseline Measures of Peak Twitch Torque (PT), Maximum Rate of Evoked Torque Development (MRTD), Time to Peak Torque (TPT), Half Relaxation Time (HRT), M-wave Area (Mwarea), M-wave Amplitude (MWamp) and M-wave Duration (MWdur).

\begin{tabular}{|c|c|c|c|c|}
\hline & $\begin{array}{c}\text { Young Males } \\
n=12\end{array}$ & $\begin{array}{c}\text { Young Females } \\
n=12\end{array}$ & $\begin{array}{c}\text { Elderly Males } \\
n=12\end{array}$ & $\begin{array}{c}\text { Elderly Females } \\
n=12\end{array}$ \\
\hline $\begin{array}{c}\text { PT } \\
(\mathrm{Nm})\end{array}$ & $0.99 \pm 0.21$ & $0.71 \pm 0.12$ & $0.73 \pm 0.24$ & $0.48 \pm 0.07$ \\
\hline $\begin{array}{l}\text { MRTD } \\
(\mathrm{Nm} / \mathrm{s})\end{array}$ & $25.8 \pm 5.4$ & $18.7 \pm 3.2$ & $20.4 \pm 6.5$ & $12.6 \pm 2.2$ \\
\hline $\begin{array}{l}\text { TPT } \\
\text { (ms) }\end{array}$ & $59.9 \pm 6.1$ & $63.2 \pm 7.2$ & $62.6 \pm 6.2$ & $60.4 \pm 7.3$ \\
\hline $\begin{array}{l}\text { HRT } \\
\text { (ms) }\end{array}$ & $70.6 \pm 9.1$ & $77.5 \pm 17.2$ & $67.1 \pm 10.8$ & $80.1 \pm 17.3$ \\
\hline $\begin{array}{c}\text { MWarea } \\
(\mu \mathrm{V} \cdot s)\end{array}$ & $79.9 \pm 10.4$ & $85.0 \pm 17.7$ & $53.9 \pm 12.4$ & $57.0 \pm 20.8$ \\
\hline $\begin{array}{c}\text { MWamp } \\
(\mathrm{mV})\end{array}$ & $20.0 \pm 2.6$ & $19.4 \pm 3.2$ & $13.3 \pm 4.4$ & $12.2 \pm 3.4$ \\
\hline $\begin{array}{c}\text { MWdur } \\
\text { (ms) }\end{array}$ & $21.1 \pm 2.3$ & $23.4 \pm 5.6$ & $20.2 \pm 2.0$ & $22.1 \pm 4.2$ \\
\hline
\end{tabular}




\subsubsection{Maximal Voluntary Contractions}

There was an age by gender interaction found for voluntary strength. Young males were significantly stronger than young females $(10.0 \pm 2.7 \mathrm{Nm}$ vs. $6.6 \pm 1.1 \mathrm{Nm}$, respectively), but there was no significant strength difference between the elderly men and women $(6.8 \pm 1.4 \mathrm{Nm}$ vs. $5.7 \pm 1.5 \mathrm{Nm}$, respectively)(Table $1 \mathrm{~b})$. This interaction was also reflected by the fact that young men were found to be stronger than elderly men, while there was no significant strength difference between the young and elderly women. These interactions persisted after controlling for the different moment arm (proximal phalanx of the thumb) lengths between subjects.

\subsubsection{Average Voluntary EMG}

There were no main effects found for either age or gender on AEMG and there was no age by gender interaction (Table $1 b)$.

\subsubsection{Motor Unit Activation}

There were no main effects found for either age or gender on the ability to activate the adductor pollicis muscle. There was also no age by gender interaction (Table $1 b)$.

\subsubsection{Estimated Adductor Pollicis Length}

There was a main effect found for both age and gender on the estimated length of the adductor pollicis muscle. The estimated length of the adductor pollicis was greater in 
males as compared to females $(3.9 \pm 0.5 \mathrm{~cm}$ vs. $3.6 \pm 0.3 \mathrm{~cm}$, respectively)(Table $1 \mathrm{~b})$, and greater in the young as compared to the elderly $(3.9 \pm 0.5 \mathrm{~cm}$ vs. $3.6 \pm 0.4 \mathrm{~cm}$, respectively)(Table $1 b)$. There was no age by gender interaction for this measure.

Table 1b. Baseline Measures of Maximum Voluntary Contractions (MVC), Average EMG (AEMG), Percent Motor Unit Activation (MUA) and Estimated Adductor Pollicis Length (AP).

\begin{tabular}{ccccc}
\hline & $\begin{array}{c}\text { Young Males } \\
\mathrm{n=12}\end{array}$ & $\begin{array}{c}\text { Young Females } \\
\mathrm{n=12}\end{array}$ & $\begin{array}{c}\text { Elderly Males } \\
\mathrm{n=12}\end{array}$ & $\begin{array}{c}\text { Elderly Females } \\
\mathrm{n}=12\end{array}$ \\
\hline $\begin{array}{c}\text { MVC } \\
(\mathrm{Nm})\end{array}$ & $10.0 \pm 2.7$ & $6.6 \pm 1.1$ & $6.8 \pm 1.4$ & $5.7 \pm 1.5$ \\
$\begin{array}{c}\text { AEMG } \\
(\mathrm{mV})\end{array}$ & $0.27 \pm 0.06$ & $0.29 \pm 0.12$ & $0.20 \pm 0.05$ & $0.26 \pm 0.13$ \\
\%MUA & $90.8 \pm 13.0$ & $80.0 \pm 11.2$ & $82.2 \pm 11.5$ & $82.9 \pm 13.5$ \\
$\begin{array}{c}\text { AP } \\
(\mathrm{cm})\end{array}$ & $4.1 \pm 0.6$ & $3.8 \pm 0.3$ & $3.7 \pm 0.4$ & $3.5 \pm 0.3$ \\
\hline $\begin{array}{l}\text { Values are means } \\
\text { (SD }\end{array}$ & & &
\end{tabular}

Values are means \pm SD.

\subsubsection{Fatigue and Recovery Measures}

As mentioned above, the measures during the fatigue protocol correspond to approximately every third contraction. Further, all mechanical and electrical variables were measured and statistically analyzed for the recovery period. However, the theoretical significance of these latter results are compromised as not all groups began the recovery period at the same level of fatigue. As well, no significant interactions of interest were found during recovery. The majority of the recovery data has therefore, not been included in this report. 


\subsubsection{The Evoked Twitch}

There was a significant 3-way, age by gender by time interaction for the fatigue of the evoked twitch torques $(p<0.001)$ which suggests that the gender differences in the reduction of peak torque depend on age. Analysis of the young males versus young females, showed a main effect for time $(p<0.001)$, with no gender by time interaction (Fig. 2a), and post hoc analysis showed that both young men and young women had significant reductions in evoked twitch torque as compared to baseline. The similar rate of fatigue between the young males and young females is also shown in their respective fatigue indices (young men: $39.8 \pm 26.7 \%$, young women: $36.6 \pm 19.0 \%$; $>0.05$ )(Table 2). Analysis of the elderly men versus elderly women, showed a gender by time interaction which suggests a gender difference in fatigability in the elderly. However, post hoc analysis revealed that there was no significant reduction of the evoked twitch from baseline, in either elderly males or elderly females, and the gender by time interaction was due to a greater twitch potentiation in elderly males (Fig. 2b). The similar rate of fatigue between the elderly males and elderly females is also shown by their respective fatigue indices (elderly men: $24.9 \pm 26.6 \%$, elderly women: $16.4 \pm$ $48.9 \% ; \mathrm{p}>0.05)$ (Table 2).

As stated above, there was no significant gender difference in the fatigue index in either age group, and furthermore, the gender by time interaction in the elderly was only due to differences in potentiation. Therefore, with respect to the fatigue index, age groups can be collapsed across gender. In this case, the fatigue index of the young tended to be greater than the fatigue index of the elderly $(38.1 \pm 22.5 \%$ vs. $20.6 \pm 40.0 \%$, 
respectively) $(\mathrm{p}=0.08)$.

There were no significant interactions found for the recovery of the evoked twitch force. Young males and females recovered to baseline force by $1 \mathrm{~min} .30 \mathrm{~s}$. while elderly males and females had recovered to baseline force by $30 \mathrm{~s}$.

Table 2. Fatigue Index of Evoked Twitch Torque (FI-PT) and Fatigue Index of Maximal Voluntary Contractions (FI-MVC).

\begin{tabular}{ccccc}
\hline & $\begin{array}{c}\text { Young Males } \\
\mathrm{n}=10\end{array}$ & $\begin{array}{c}\text { Young Females } \\
\mathrm{n}=11\end{array}$ & $\begin{array}{c}\text { Elderly Males } \\
\mathrm{n}=10\end{array}$ & $\begin{array}{c}\text { Elderly Females } \\
\mathrm{n}=9\end{array}$ \\
\hline $\begin{array}{c}\text { FI-PT } \\
(\%)\end{array}$ & $39.8 \pm 26.7$ & $36.6 \pm 19.0$ & $24.9 \pm 26.6$ & $16.4 \pm 48.9$ \\
$\begin{array}{c}\text { FI-MVC } \\
(\%)\end{array}$ & $44.7 \pm 10.5$ & $37.8 \pm 14.1$ & $24.2 \pm 10.7$ & $26.3 \pm 14.5$ \\
\hline
\end{tabular}

Values are means \pm SD.

\subsubsection{Maximum Rate of Evoked Torque Development}

There was a significant 3-way, age by gender by time interaction for the MRTD $(p<0.01)$, which suggests that gender differences in the reduction of MRTD depend on age. Post hoc analysis showed that the MRTD in young males decreased significantly below baseline in the latter stages of the fatigue protocol, while the MRTD in young females only showed a trend for a reduction during the final two contractions $(p=0.09$, $\mathrm{p}=0.06$ ); whereas neither the elderly males nor the elderly females showed significant reductions in MRTD throughout the fatigue protocol (Fig. 3a,b). Also contributing to the 3-way interaction was the fact that both young men and young women showed a significant potentiation of MRTD, whereas in the elderly, only the men showed this MRTD potentiation (Fig. 3a,b). 


\subsubsection{Half-Relaxation Time}

Analysis of young males versus elderly males, showed a main effect for time $(\mathrm{p}<0.01)$, with no significant age by time interaction. Likewise, analysis of young females versus elderly females, showed a trend for time $(p=0.06)$, with no significant age by time interaction. The male and female groups have therefore, been collapsed across age. The resulting analysis revealed a significant gender by time interaction $(p<0.01)$ for HRT of the evoked twitch during fatigue. Post hoc analysis showed that there was no significant change in the female HRT relative to baseline, while the HRT in males increased, and at approximately 2 minutes, HRT was significantly longer than at baseline as well as significantly longer compared to the corresponding HRT in females (Fig. 4). In the latter stages of the fatigue protocol, however, the HRT in males decreased, and returned to values similar to those at baseline.

\subsubsection{M-wave Area}

Analysis of all four groups together, showed a significant age by time interaction for the behavior of the $\mathrm{M}$-wave area during fatigue $(\mathrm{p}<0.01)$. Post hoc analysis revealed that the elderly (collapsed across gender), had a significant potentiation of the M-wave area early into the fatigue protocol, with no significant reduction of the M-wave area relative to baseline at any time (Fig. 5b). The young (collapsed across gender), showed no significant change in M-wave area, relative to baseline, at any time. However, further analysis revealed a gender by time interaction in the young men and women, and 
therefore, they should not be collapsed into one group. Analysis of young men, as a single group, revealed a significant main effect for time $(p<0.01)$, with a trend toward reduction of the $\mathrm{M}$-wave area at the end of the fatigue protocol $(\mathrm{p}=0.08)$ (Fig 5a). Young women, however, showed no main effect for time, and no significant change in M-wave area at any point throughout the fatigue protocol (Fig. 5a). Analysis of the elderly men and elderly women, as single groups, still showed no significant reduction of M-wave area relative to baseline, at any time for either gender.

\subsubsection{M-wave amplitude}

The behavior of the M-wave amplitude was similar to that of the M-wave area, i.e. analysis of all groups together showed a significant age by time interaction $(p<0.001)$, despite a significant gender by time interaction amongst the young $(\mathrm{p}<0.001)$. Therefore, for the reasons stated previously, the young males and young females will be considered separately. Young males showed a significant reduction of the M-wave amplitude relative to baseline, by approximately 2 minutes, while young females showed no significant reduction of the M-wave amplitude at any time (Fig. 6a). There was no significant gender by time interaction amongst the elderly males and females, and collapsed into one group, the elderly showed a significant potentiation of the M-wave amplitude, followed by a significant reduction of the $\mathrm{M}$-wave amplitude, relative to baseline, after 3 minutes (Fig. 6b). 


\subsubsection{M-Wave Duration}

Analysis of all groups together, showed a significant main effect for time $(\mathrm{p}<0.001)$, with no 2-way or 3-way interactions, and therefore, all subjects may be collapsed into one group. Post hoc analysis showed that the M-wave duration showed an initial significant reduction at approximately 25 seconds, and although it continued to rise throughout the fatigue protocol, the M-wave duration never reached values that were significantly greater than those at baseline (Fig. 7).

\subsubsection{Maximum Voluntary Contractions}

Analysis of young males versus young females showed a strong trend toward a gender by time interaction $(p=0.06)$, which suggests that young males tended to fatigue more than young females. This difference is also reflected by the fatigue index, although it did not reach statistical significance (young males: $44.7 \pm 10.5 \%$ vs. young females: $37.8 \pm 14.1 \% ; \mathrm{p}=0.12)$ (Table 2). Post hoc analysis of the time main effect, showed that significant reductions in voluntary torque were apparent by approximately 45 seconds in both young males and young females (Fig. 8a).

Analysis of elderly males versus elderly females, showed no gender by time interaction, which suggests a similar amount of fatigue in elderly men and women (Fig. 8b). The fatigue index also showed no significant gender difference in the fatigability of the elderly (elderly males: $24.2 \pm 10.7 \%$ vs. elderly females: $26.3 \pm 14.5 \%$; $\mathrm{p}>0.05)($ Table 2). Post hoc analysis showed that the elderly had significant reductions of voluntary force by approximately 2 minutes 30 seconds. 
Collapsing across gender resulted in a significant age by time interaction $(\mathrm{p}<0.001)$, which suggests that the young fatigue more than the elderly. However, further analysis showed that although there was a highly significant difference between the fatigue indices of young and elderly males $(\mathrm{p}<0.01)$ (Table 2, Fig. 8c), there was no significant difference between the fatigue indices of young and elderly females $(p=0.13$ ) (Table 2, Fig. 8d). Therefore, while young males were more fatigable than elderly males, young females and elderly females showed a similar fatigability.

After 10 minutes of recovery all groups had returned to baseline voluntary strength.

\subsubsection{AEMG}

Although analysis of all groups together showed a small but significant age by time interaction for AEMG during fatigue $(\mathrm{p}=0.04)$, post hoc analysis revealed that neither the young nor the elderly had significant reductions of AEMG, relative to baseline, at any time during the fatigue protocol (Fig. 9).

\subsubsection{Motor Unit Activation}

Analysis of young males versus young females, showed a main effect for time $(p<0.001)$, with no significant gender by time interaction, and post hoc analysis showed that both young males and young females had significant reductions in MUA, relative to baseline, in the final stages of the fatigue protocol. Analysis of elderly males versus elderly females, showed no significant gender by time interaction, and post hoc analysis of the time main effect showed that the elderly subjects experienced no significant 
reduction in MUA at any time during the fatigue protocol. When collapsed across gender, there was a significant age by time interaction for MUA $(p<0.001)$, such that the young showed a decrease in muscle activation with time while the elderly showed so such change (Fig. 10). The validity of this measure, however, may be suspect (see discussion).

\subsubsection{The AEMG:M-wave Area Ratio}

When collapsed across gender, there was a significant age by time interaction $(\mathrm{p}<0.01)$, for the AEMG:M-wave area ratio, however, post hoc analysis showed that neither the young nor the elderly had any significant change from baseline in this ratio at any time (Fig. 11).

\subsubsection{Adductor Pollicis Length and Absolute Baseline Force}

Multiple regression analysis was performed in order to determine the influence that adductor pollicis length and absolute baseline force had on relative fatigability. When each group was considered separately, neither adductor pollicis length, nor absolute baseline force, accounted for a significant portion of the variance in the voluntary fatigue index in any group.

When the young were considered as one group, gender was found to account for $12.29 \%$ of the variability in the voluntary fatigue index $(\mathrm{p}=0.12)$. Absolute baseline force was then added to the regression equation and the two variables together accounted for $13.78 \%$ of the variability in the fatigue indices of the young. Absolute baseline force, 
therefore, did not significantly add to the accounted for variability in the voluntary fatigue indices in the young $(\mathrm{p}=0.58)$. As well, the addition of adductor pollicis length to the regression equation, did not significantly increase the amount of accounted for variability in voluntary fatigue in the young i.e. the three variables together, accounted for $13.8 \%(p=0.93)$.

As there was not a significant amount of variability between elderly males and elderly females, with respect to the fatigue index, regression analysis was not performed on the elderly as a group.

When males were considered as one group, age was found to account for $52.15 \%$ of the variability in the voluntary fatigue index $(\mathrm{p}<0.001)$. Absolute force was then added to the regression analysis and the two variables together accounted for $53.76 \%$ of the variability in voluntary fatigue. Therefore, absolute force did not significantly add to the accounted for variability in voluntary fatigue between young and elderly males $(p=0.45)$. Adductor pollicis length was then added to the regression equation and the three variables together accounted for $53.76 \%$ of the variability in the voluntary fatigue index. Therefore, adductor pollicis length did not significantly add to the accounted for variability in the voluntary fatigue indices of males $(\mathrm{p}=0.98)$.

As there was not a significant amount of variability between young and elderly females, with respect to the fatigue index, regression analysis was not performed on the female group.

A final regression analysis was performed with all subjects pooled and with consideration for all four variables (age, gender, absolute baseline force and adductor 
pollicis length). Age was the only variable which accounted for a significant proportion of the variability in the fatigue indices of all subjects together $(p<0.004)$. This suggests that, considering all four variables, age was the strongest predictor of fatigability of the human adductor pollicis muscle.

\section{$2.4 \quad$ Discussion}

\subsubsection{Gender Differences in Fatigue in Young Adults}

Young males and young females showed no gender difference in relative fatigability during the 3 minute fatigue protocol, as indicated by their respective reductions in evoked twitch torque and calculated fatigue indices. The evoked twitch measures, however, may have been confounded by gender differences in post-activation potentiation, and therefore, should be interpreted with caution. Therefore, examination of the voluntary force measures may be a more suitable way to investigate the possible gender differences in the fatigability of the young.

In contrast to the evoked twitch measures, the MVC data revealed a strong trend for young males to be more fatigable than young females $(p=0.06)$. The more profound gender differences in fatigue that have been shown by others (Lewis et al., 1997; West et al., 1995), may be due to either methodological differences from the present study, or by differing definitions of fatigue. For example, Lewis et al. (1997), employed an intermittent submaximal fatigue protocol and used a temporally based definition of fatigue, i.e. the time at which $50 \%$ of the MVC could no longer be produced. As a 
result, Lewis' fatigue protocol was much longer than that of the present study (approximately 14 minutes for females), and the well established female advantage in aerobic metabolism (Tarnopolsky et al., 1990; Tarnopolsky et al., 1995) may have been largely responsible for the gender difference that Lewis and colleagues found. In the present study, females would not have benefited as much from their more efficient aerobic metabolism of $\mathrm{CHO}$. Further, the submaximal contractions used in the studies by Lewis et al. (1997) and West et al. (1995) may have allowed for greater degrees of muscular blood flow than the maximal contractions used in the present study. If young females do benefit from a more favorable blood supply to exercising muscles as compared to males, then their advantage in muscular endurance would be more noticeable during submaximal contractions. It is interesting to note that Maughan et al. (1986) found the isometric endurance times of males to be significantly shorter than females during contractions at $20 \%$ of the MVC, but found no gender difference in fatigability during contractions at 50 or $80 \%$ of the MVC. The gender difference in fatigue shown in the West study (1995) also seemed to be inversely proportional to contraction intensity; i.e. men exercised approximately $68 \%$ as long as women at $50 \%$ MVC, but were able to exercise $74 \%$ as long as women at $75 \%$ MVC.

There may have been many interacting mechanisms contributing to the gender difference in fatigue in the present study. With respect to neuromuscular transmission failure, young males showed slight, but significant, decreases in M-wave amplitude and a trend for a decrease in M-wave area, while young females did not. These data may suggest that young males were susceptible to either transmission failure at the 
neuromuscular junction, a decrease in muscle membrane excitability, or a combination of the two. Reductions in AEMG would be expected to accompany reductions in M-wave amplitude and area, and while young males showed slight decreases in AEMG, this measure did not reach statistical significance. Regardless, the maintenance of the AEMG:M-wave area ratio in young males, suggests that this group was not experiencing central fatigue. The fact that young females showed a significant degree of fatigue despite the maintenance of the $\mathrm{M}$-wave amplitude, $\mathrm{M}$-wave area and AEMG, suggests that the site of fatigue was distal to sarcolemmal action potential propagation. As stated previously, however, the behavior of the M-wave during voluntary fatigue is controversial. Merton (1954), found no reduction of the M-wave amplitude, despite the substantial decline in force that resulted from 3 minutes of maximal adductor pollicis contractions. In contrast, reductions in the M-wave amplitude were found to accompany fatigue of the adductor pollicis in a study conducted by Bellemare and Garzaniti (1988). Unfortunately, neither study reported the genders of their subjects. Studies by BiglandRitchie et al. (1986), and Hicks et ail. (1992), both found a maintenance of the M-wave amplitude during muscle fatigue in groups containing both males and females, however, no gender comparisons were made. In the present study, the differences in M-wave behavior between males and females were relatively minor, with statistical significance only evident in the M-wave amplitude measurement.

Many mechanisms may have contributed to the maintenance of neuromuscular transmission in females. It is possible that a gender difference may exist in the concentration or activity of the $\mathrm{Na}^{+} / \mathrm{K}^{+}$ATPase enzyme, although the present study did 
not provide any direct evidence to support this theory. It is also possible that females benefit from a more favorable blood supply to exercising muscles as compared to males, which would more effectively clear extracellular potassium during exertion. Although the maximal contractions performed in the present study may seem to preclude muscular blood flow, this may not have been the case. The alternating 2 second rest periods would have allowed blood to reach the exercising muscles, and further, as time progressed both males and females were performing contractions at a progressively lower percentage of their baseline MVC. At these lower absolute forces blood flow may not have been occluded. The gender by time interaction found for HRT, may also support the theory of a more favorable blood flow in young females as compared to young males. To expand, it has been frequently observed that lactic acidosis increases HRT, and that the muscle fibres which show the largest increases in $\left[\mathrm{H}^{+}\right]$, also show the greatest prolongation of relaxation (for a review see Fitts, 1994). The maintenance of HRT in young females may therefore be indicative of either a lower production, or a more effective clearance of lactic acid as compared to young males. Furthermore, assuming that the vascular clearance of $\mathrm{K}^{+}$parallels the vascular clearance of $\mathrm{H}^{+}$, young females may also have more effectively cleared extracellular $\mathrm{K}^{+}$, thereby maintaining a greater membrane excitability than young males, secondary to a possible advantage in muscular blood flow. Whatever the mechanisms that underlie the female advantage in membrane excitability maintenance, they seem to be estrogen-based, as elderly females showed similar decreases in the $\mathrm{M}$-wave amplitude as compared to elderly males. 
Fibre type differences in the adductor pollicis between young males and young females may also have contributed to the observed gender difference in fatigue. If young males did in fact possess a higher proportion of Type II muscle fibres, as some have suggested (Miller, 1993; Simoneau and Bouchard, 1989), they would have been more prone to fatigue as a result of both a greater lactic acid accumulation than that in young females, and possibly a higher degree of muscular blood flow occlusion due to the greater specific forces that Type II fibres may exert as compared to Type I fibres (Grindrod et al., 1987; Young, 1984). The significantly greater baseline MRTD in young males as compared to young females provides some support for a possible gender difference in fibre type distribution. Moreover, the gender by time interaction in HRT may again be reflective of a lower lactic acid accumulation in young females as compared to young males, which may be secondary to a lower proportion of Type II fibres in the former.

In the present study, multiple regression analysis showed that gender tended to account for the greatest portion of the variability in fatigue in the young. However, neither absolute baseline force nor the number of sarcomeres in series, as estimated by adductor pollicis length, were found to significantly add to the accounted for variability in fatigue in the young. The former finding is in agreement with Lewis and colleagues (1997), while the latter is in conflict with the hypothesis put forth by de Haan et al. (1988). However, as our estimate of the number of sarcomeres in series was crude at best, this result should be interpreted with caution, and therefore, more research is required to adequately test de Haan's theory. 


\subsubsection{Gender Differences in Fatigue in the Elderly}

A gender by time interaction was found between elderly males and elderly females in the evoked twitch measures, however, this relationship was due to differences in post-activation potentiation, rather than differences in fatigability per se. Therefore, the MVC measures may give a better depiction of the relative fatigability between elderly males and elderly females. With respect to the voluntary contractions, there was no gender difference in fatigue resistance between these two groups as indicated by the lack of a gender by time interaction during the fatigue protocol, as well as the lack of a significant difference between the fatigue indices of elderly males and elderly females at the conclusion of the fatigue protocol. These results appear to be in conflict with the findings of Hicks and McCartney (1996), who found elderly females to be significantly more fatigue resistant that elderly males during elbow flexor and ankle dorsiflexor exercise. It is possible that methodological differences between these two studies account for the discrepancy in the findings. Although Hicks and McCartney used an identical fatigue protocol as that of the present study ( 3 minutes of intermittent MVC's; $5 \mathrm{~s}$ contractions, $2 \mathrm{~s}$ rest), the difference in the muscle groups examined may have had profound effects. In young subjects, the adductor pollicis muscle has been found to consist of approximately $80 \%$ Type I fibres, while the ankle dorsiflexors and elbow flexors have been shown to contain approximately $70-75 \%$ and $40-65 \%$ Type I fibres, respectively (Johnson et al., 1973). Because aging is associated with a loss of both Type I and Type II fibres, but a selective atrophy of Type II fibres, the elderly may not maintain enough absolute Type II muscle mass in the adductor pollicis to demonstrate a 
gender difference in fatigue in this muscle. In other words, the age-related atrophy of Type II fibres may eliminate the gender differences in fatigue in predominantly Type I muscles, such as the adductor pollicis, but not eliminate the gender difference in fatigue in mixed muscle, such as the ankle dorsiflexors and especially the elbow flexors, in which a greater absolute amount of preserved Type II muscle mass would be expected. This theory is consistent with the fact that Hicks and McCartney (1996) seemed to find a more pronounced gender difference in fatigue in the elbow flexors of the elderly (male fatigue index: $64.44 \pm 16.65 \%$ vs. female fatigue index: $38.74 \% \pm 20.51 \%$ ), than in the ankle dorsiflexors of the elderly (male fatigue index: $67.07 \% \pm 23.72 \%$ vs. female fatigue index: $53.83 \% \pm 27.56 \%)$.

It may also be the case that distal muscles such as the adductor pollicis, may experience different age-related changes than more proximal muscles such as the biceps. For example, Galea (1996), found evidence for denervation and reinnervation in the thenar muscles of the elderly but not in the biceps. The type I fibre grouping that results from denervation/reinnervation may have eliminated gender differences in fatigue in the elderly adductor pollicis muscle, whereas the elderly biceps may have maintained a gender difference in fibre type and fatigability.

In the present study elderly males and elderly females showed small but significant reductions of the M-wave amplitude, which suggests both groups were susceptible to impulse transmission failure at the neuromuscular junction and/or along the muscle membrane. As stated previously, exercise-induced reductions in AEMG would be expected to accompany exercise-induced reductions in the M-wave amplitude, 
although this was not the case in the present study. Nevertheless, the maintenance of MUA in the elderly subjects, suggests that the aged were not susceptible to central fatigue.

An interesting finding in the present study was that both elderly males and elderly females showed a significant potentiation of the M-wave amplitude and area early in the fatigue protocol. While this enlargement of the M-wave has been previously shown in the adductor pollicis (Duchateau and Hainaut, 1985) and thenar muscles (Hicks et al. 1989a) of young adults, it has not been previously shown in the elderly. Possible mechanisms accounting for this $\mathrm{M}$-wave potentiation include a greater synchronization of muscle fibre action potentials (Fitch and McComas, 1985) or a hyperpolarization of the muscle membrane caused by a contraction-induced increase in the activity of the $\mathrm{Na}^{+} / \mathrm{K}^{+}$ATPase enzyme (Hicks et al., 1989b). The concomitant decrease in M-wave duration that occurred in the present study suggests that the former mechanism may have accounted, in part, for the observed M-wave potentiation. Whether or not membrane hyperpolarization also contributed to the enlargement of the M-wave can not be concluded from the present study. It is interesting to note, however, that in elderly females the enlargement of the M-wave occurred in the absence of any twitch torque potentiation. This finding helps to confirm the conclusions of Hicks et al. (1989a), who determined that " $\mathrm{M}$-wave enlargement cannot be an artifact related to muscle contractions." 


\subsubsection{Age-related differences in fatigue}

Examination of the voluntary fatigue index showed no significant age-related difference in the fatigue resistance between young females and elderly females, despite the apparent difference in the means $(37.8 \pm 14.1 \%$ vs $26.3 \pm 14.5 \%$, respectively) . However, there was a significant age-related difference in the fatigability of men, such that elderly males were distinctly more fatigue resistant that young males. It is difficult to specify the cause of this gender disparity as there is such a variety of age-related changes that occur in human skeletal muscle. It can be speculated however, that as females age they experience the concomitant effects of a loss of estrogen, which may hinder fatigue resistance, together with an increase in the proportion of their Type I muscle fibres, which would enhance fatigue resistance. On the other hand, as males age, their muscular endurance may predominantly benefit from histological changes alone.

The age-related enhancement in fatigue resistance that was found for males in the present study is in conflict with some of the other work in this area. However, as stated previously, those who have shown age-related decrements in fatigue resistance have used electrically-induced fatigue protocols, without correcting for the age-related leftward shift in the force-frequency relationship (Cupido et al., 1992; Davies and White, 1983; Davies et al., 1986), and as a result, may have underestimated the muscular endurance of the elderly. The results of the present study are also in conflict with those who have used voluntary fatigue protocols to examine the age-related changes in muscular endurance. For example, elderly males and young males have been shown to have similar endurance times during sustained handgrip contractions at 40\% MVC (Petrofsky and Lind, 1975), as 
well as during sustained quadriceps contractions at 50\% MVC (Larsson and Karlsson, 1978). However, methodological differences between these two studies and the present one, may explain the disparity in the results. Specifically, the submaximal fatigue protocols that were used in the above studies may not have stressed the Type II muscle fibres to the same extent as the maximal contractions that were used in the present study. This suggests that an age-related reduction in the proportion of Type II muscle fibres may in fact enhance the fatigue resistance of elderly males, compared to young males, as the age-related difference in fatigue seems to be most pronounced during high intensity fatigue protocols. Further support for this theory is provided by the fact that the elderly males in the present study had significantly slower MRTD than the young males, which would be indicative of a smaller proportion of Type II muscle fibres.

Finally, it must be clarified that the age-related difference in fatigue resistance was not caused by the fact that young males and females showed a significant reduction in MUA late in the fatigue protocol, while elderly males and females did not. The agerelated difference in fatigability occurred well before any differences were noted in the degree of MUA. In addition, the reductions that were found in MUA in young males and females should not be interpreted as signs of either central fatigue or a lack of motivation. Rather, it was common for the young subjects to inadvertently augment their strength late in the fatigue protocol by recruiting their median-supplied thenar muscles. Despite the palm-down position of the hand, which was meant to discourage force production from the thumb flexors and opponens muscle, a slight external rotation of the hand was possible. In this position, the thenar muscles became active and the adductor pollicis 
somewhat relaxed. This resulted in an exaggerated interpolated twitch and an underestimated MUA. While the elderly subjects maintained the palm-down position throughout the fatigue protocol, the young subjects were more prone to sacrifice proper form for the sake of augmented force.

\subsubsection{Conclusions}

To summarize the gender differences in fatigue, young males demonstrated a strong tendency to be more fatigable than young females, based on the voluntary force measures $(\mathrm{p}=0.06)$. There was no significant gender difference in fatigue between elderly males and elderly females. The fact that a gender difference in muscular endurance was found in the young but not in the elderly, suggests that estrogen possesses fatigue resisting properties even in exercise of short duration. Nevertheless, the lack of a significant gender difference in fatigue between elderly males and elderly females may be specific to the adductor pollicis muscle, and more research will be required to settle this issue.

To summarize the effects of aging on fatigability, elderly males were found to be distinctly more fatigue resistant than young males, and while a similar trend existed between young and elderly females, it did not attain statistical significance. This apparent endurance advantage in elderly men may have resulted from a lower proportion of Type II fibres in the elderly males, as compared to the young males. While elderly females may also benefit from a lower proportion of Type II fibres, their age-related loss of estrogen may offset any endurance advantage, as compared to young females. 
In conclusion this study was designed to determine the relative influence of factors such as age, gender, absolute muscle force and estimated muscle length on fatigability of the adductor pollicis muscle. While age was found to be the strongest single predictor of fatigability when all subjects were pooled together, gender also seemed to play a role in determining endurance during short-term fatigue paradigms in young adults. 


\section{$\underline{\text { References }}$}

Aniansson, A., Grimby, G., Hedberg, M., Rundgren, A. and Sperling, L. (1978). Muscle function in old age. Scand. J. Rehab. Med. (Suppl.) 6: 43-49.

Belanger, A.Y. and McComas, A.J. (1981). Extent of motor unit activation during effort. J. Appl. Physiol. 51(5): 1131-1135.

Bellemare, F. and Garzaniti, N. (1988). Failure of neuromuscular propagation during human maximal voluntary contraction. J. Appl. Physiol. 64(3): 1084-1093.

Bigland-Ritchie, B., Furbush, F. and Woods, J.J. (1986). Fatigue of intermittent submaximal voluntary contractions: central and peripheral factors. J. Appl. Physiol. 61(2): 421-429.

Bruce, S.A., Newton, D. and Woledge, R.C. (1989). Effect of age on voluntary force and cross-sectional area of human adductor pollicis muscle. Q. J. Exp. Physiol. 74, 359-362.

Burke, W.E., Tuttle, W.W., Thompson, C.W., Janney, C.D. and Weber, R.J. (1953). The relation of grip strength and grip strength endurance to age. J. Appl. Physiol. 5: 628-630.

Campbell, M.J., McComas, A.J. and Petito, F. (1973). Physiological changes in aging muscles. J. Neurol. Neurosurg. Psychiatry 36:174-182.

Collumbine, H., Bibile, S.W., Wikramanayake, T.W. and Watson, R.S. (1950). Influence of age, sex, physique and muscular development on physical fitness. J. Appl. Physiol. 2: 488-511.

Cupido, C.M., Hicks, A.L. and Martin, J. (1992). Neuromuscular fatigue during repetitive stimulation in elderly and young adults. Eur. J. Appl. Physiol. 65: 567-572.

Davies, C.T.M., White, M.J. and Young, K. (1983). Electrically evoked and voluntary maximal isometric tension in relation to dynamic muscle performance in elderly male subjects, aged 69 years. Eur. J. Appl. Physiol. 51: 37-43.

Davies, C.T.M., Thomas, D.O. and White, M.J. (1986). Mechanical properties of young and elderly human muscle. Acta Med. Scand. (Suppl.) 711:219-226.

de Haan, A., Rexwinkel, R., van Doorn, J., Westra, H., Hollander, A., Huijing, P., Woittiez, R. and Sargeant, A. (1988). Influence of muscle dimensions on economy of isometric exercise in rat medial gastrocnemius muscles in situ. Eur. J. Appl. Physiol. 57: 64-69. 
Duchateau, J. and Hainaut, K. (1985). Electrical and mechanical failures during sustained and intermittent contractions in humans. J. Appl. Physiol. 58(3): $942-$ 947.

Fitch, S. and McComas, A.J. (1985). Influence of human muscle length on fatigue. J. Physiol. 362: 205-213.

Fitts, R.H. (1994). Cellular mechanisms of muscle fatigue. Physiological Reviews. 74(1): 49-94.

Galea, V. (1996). Changes in motor unit estimates with aging. J. Clin. Neurophysiol. 13(3): 253-260.

Govindasamy, D. and Paterson, D. (1994). The physiology of aging. In: Zeigler, E., editor. Physical activity and the older adult: A knowledge base for managing exercise programs. Illinois: Stipes Publishing Company, 2-5.

Grindrod, S., Round, J.M. and Rutherford, O.M. (1987). Type 2 fibre composition and force per cross-sectional area in the human quadriceps (Abstract). J. Physiol. 390: $154 \mathrm{P}$.

Hakkinen, K. and Hakkinen, A. (1991). Muscle cross-sectional area, force production and relaxation characteristics in women at different ages. Eur. J. Appl. Physiol. 62: 410-414.

Hicks, A.L. and McComas, A.J. (1989a). Increased sodium pump activity following stimulation of rat soleus muscles. J. Physiol. 414: 337-349.

Hicks, A.L., Fenton, J., Garner, S. and McComas, A.J. (1989b). M wave potentiation during and after muscle activity. J. Appl. Physiol. 66(6): 2606-2610.

Hicks, A.L., Cupido, C.M., Martin, J. and Dent, J. (1992). Muscle excitation in elderly adults: The effects of training. Muscle Nerve 15: 87-93.

Hicks, A. and McCartney, N. (1996). Gender Difference in isometric contractile properties and fatigability in elderly human muscle. Can. J. Appl. Phys. 21(6): 441-454.

Houston, M.E., Green, H.J. and Stull, J.T. (1985). Myosin light chain phosphorylation and isometric twitch potentiation in intact human muscle. Pflugers Arch. 403: 348-352. 
Johnson, M.A., Polgar, D., Weightman, D. and Appleton, D. (1973). Data on the distribution of fibre types in thirty-six human muscles: An autopsy study. J. Neurol. Sci. 18: 111-129.

Kendrick, Z.V., Steffen, C.A., Rumsey, W.L. and Goldberg, D.I. (1987). Effect of estradiol on tissue glycogen metabolism in exercised oophorectomized rats. J. Appl. Physiol. 63(2): 492-496.

Klein, C., Cunningham, D.A., Paterson, D.H. and Taylor, A.W. (1988). Fatigue and recovery contractile properties of young and elderly men. Eur. J. Appl. Physiol. 57: 684-690.

Larsson, L. and Karlsson, J. (1978). Isometric and dynamic endurance as a function of age and skeletal muscle characteristics. Acta. Physiol. Scand. 104: 129-136.

Lewis, S.F., Rock, P.B., Muza, S.R. Lammi, E., Moore, L.G., Cymerman, A. and Fulco, C.S. (1997). Slower rate of adductor pollicis muscle fatigue in women than in men. (Abstract). Med. Sci. Sport Exer. Suppl. 29(5): S92.

Lexell, J., Taylor, C. and Sjostrom, M. (1988). What is the cause of aging atrophy? Total number, size and proportions of different fibre types studied in whole vastus lateralis muscle from 15 to 83 year old men. J. Neurol. Sci. 84: 275-294.

Maughan, R.J., Harmon, M., Leiper, J.B., Sale, D. and Delman, A. (1986). Endurance capacity of untrained males and females in isometric and dynamic muscular contractions. Eur. J. Appl. Physiol. 55: 395-400.

Merton, P.A. (1954). Voluntary strength and fatigue. J. Physiol. 123: 553-564.

Miller, A.E.J., MacDougall, J.D. Tarnopolsky, M.A. and Sale, D.G. (1993). Gender differences in strength and muscle fibre characteristics. Eur. J. Appl. Physiol. 66: 254-262.

Narici, M.V., Bordini, M. and Cerretelli, P. (1991). Effect of aging on human adductor pollicis muscle function. J. Appl. Physiol. 71(4): 1277-1281.

Petrofsky, J.S. and Lind, A.R. (1975). Aging, isometric strength and endurance, and cardiovascular response to static effort. J. Appl. Physiol. 38(1): 91-95.

Rooney, T.P., Kendrick, Z.V., Carlson, J., Ellis, G.S., Matakevich, B., Lorusso, S.M. and McCall, J.A. (1993). Effect of estradiol on the temporal pattern of exerciseinduced tissue glycogen depletion in male rats. J. Appl. Physiol. 75(4): 15021506. 
Sale, D.G. (1999). Neuromuscular Function. In: Tarnopolsky, M.A., editor. Gender differences in metabolism: Practical and nutritional implications. Boca Raton, Florida: CRC Press LLC, 61-85.

Schantz, P., Randell-Fox, E., Hutchinson, W., Tyden, A. and Astrand, P.O. (1983). Muscle fibre type distribution, muscle cross-sectional area and maximal voluntary strength in humans. Acta Physiol. Scand. 117: 219-226.

Simoneau, J.A. and Bouchard, C. (1989). Human variation in skeletal muscle fibre type proportion and enzyme activities. Am. J. Physiol. 257: E567-E572.

Salmons, S. (Ed.). (1995). Muscle. In: Gray's Anatomy (38th ed.), pg. 859. New York: Churchill, Livingstone.

Tarnopolsky, L.J., MacDougall, J.D., Atkinson, S.A., Tarnopolsky, M.A. and Sutton, R.J. (1990). Gender difference in substrate for endurance exercise. J. Appl. Physiol. 68: 302-308.

Tarnopolsky, M.A., Atkinson, S.A., Phillips, S.M. and MacDougall, J.D. (1995). Carbohydrate loading and metabolism during exercise in men and women. $\mathbf{J}$. Appl. Physiol. 78: 1360-1368.

Vandervoort, A.A., Quinlan, J. and McComas, A.J. (1983). Twitch potentiation after voluntary contraction. Exp. Neurol. 81: 141-152.

Vandervoort, A.A. and McComas, A.J. (1986). Contractile changes in opposing muscles of the human ankle joint with aging. J. Appl. Physiol. 61: 361-367.

West, W., Hicks, A., Clements, L. and Dowling, J. (1995). The relationship between voluntary electromyogram, endurance time and intensity of effort in isometric handgrip exercise. Eur. J. AppI. Physiol. 71: 301-305.

Young, A. (1984). The relative isometric strength of type I and type II muscle fibres in the human quadriceps. Clin. Physiol. 4: 23-32. 


\subsection{Figure Captions}

Figure 1. Drawing of Apparatus

Figure 2a. Changes in evoked twitch torque, relative to baseline for young males $(n=10)$ and young females $(\mathrm{n}=11) .{ }^{*}$ denotes significant difference from baseline for young males $(p<0.05),+$ denotes significant difference from baseline for young females $(\mathrm{p}<0.05)$

Figure 2b. Changes in evoked twitch torque, relative to baseline for elderly males $(n=11)$ and elderly females $(n=10) .{ }^{*}$ denotes significant difference from baseline for elderly males $(\mathrm{p}<0.05)$.

Figure 3a. Changes in maximum rate of evoked torque development, relative to baseline for young males $(n=10)$ and young females $(n=11) .{ }^{*}$ denotes significant difference from baseline for young males $(\mathrm{p}<0.05)$, + denotes significant difference from baseline for young females $(\mathrm{p}<0.05)$.

Figure 3b. Changes in maximum rate of evoked torque development, relative to baseline for elderly males $(n=11)$ and elderly females $(n=10) .{ }^{*}$ denotes significant difference from baseline for elderly males $(\mathrm{p}<0.05)$. 
Figure 4. Changes in half relaxation time, relative to baseline for males $(n=21)$ and females $(n=21) .{ }^{*}$ denotes significant difference from baseline for males $(p<0.05)$, - denotes significant difference between males and females $(\mathrm{p}<0.05)$.

Figure 5a. Changes in $M$-wave area, relative to baseline for young males $(n=9)$ and young females $(n=8)$.

Figure 5b. Changes in M-wave area, relative to baseline for elderly subjects ( $n=16)$. ${ }^{*}$ denotes significant difference from baseline $(\mathrm{p}<0.05)$.

Figure 6a. Changes in M-wave amplitude, relative to baseline for young males ( $\mathrm{n}=9$ ) and young females $(n=8) .{ }^{*}$ denotes significant difference from baseline for males $(\mathrm{p}<0.05)$, $\bullet$ denotes significant difference between young males and young females $(\mathrm{p}<0.05)$.

Figure 6b. Changes in M-wave amplitude, relative to baseline for the elderly $(p=16)$.

${ }^{*}$ denotes significant difference from baseline

Figure 7. Changes in $\mathrm{M}$-wave duration, relative to baseline for all subjects ( $\mathrm{n}=33$ ).

* denotes significant difference from baseline $(\mathrm{p}<0.05)$. 
Figure 8a. Changes in maximum voluntary torque, relative to baseline for young males $(n=10)$ and young females $(n=11) .{ }^{*}$ denotes significant difference from baseline for young males $(p<0.05),+$ denotes significant difference from baseline for young females $(\mathrm{p}<0.05)$.

Figure 8b. Changes in maximum voluntary torque, relative to baseline for elderly males $(n=11)$ and elderly females $(n=10) .{ }^{*}$ denotes significant difference from baseline for elderly males $(\mathrm{p}<0.05),+$ denotes significant difference from baseline for elderly females $(\mathrm{p}<0.05)$.

Figure 8c. Changes in maximum voluntary torque, relative to baseline for young males $(n=10)$ and elderly males $(n=11) .{ }^{*}$ denotes significant difference from baseline for young males $(\mathrm{p}<0.05),+$ denotes significant difference from baseline for elderly males $(p<0.05)$, denotes significant difference between young males and elderly males $(\mathrm{p}<0.05)$.

Figure 8d. Changes in maximum voluntary torque, relative to baseline for young females $(n=11)$ and elderly females $(n=10) .{ }^{*}$ denotes significant difference from baseline for young females $(p<0.05),+$ denotes significant difference from baseline for elderly females $(\mathrm{p}<0.05)$. 
Figure 9. Changes in AEMG, relative to baseline for young subjects $(n=17)$ and elderly subjects $(n=16)$.

Figure 10. Changes in percent motor unit activation, relative to baseline for young subjects $(\mathrm{n}=18)$ and elderly subjects $(\mathrm{n}=20) .{ }^{*}$ denotes significant difference from baseline for young subjects $(\mathrm{p}<0.05), \bullet$ denotes significant difference between young and elderly subjects $(\mathrm{p}<0.05)$.

Figure 11. Changes in the AEMG:M-wave area ratio, relative to baseline for young subjects $(n=17)$ and elderly subjects $(n=16)$. 
Figure 2a

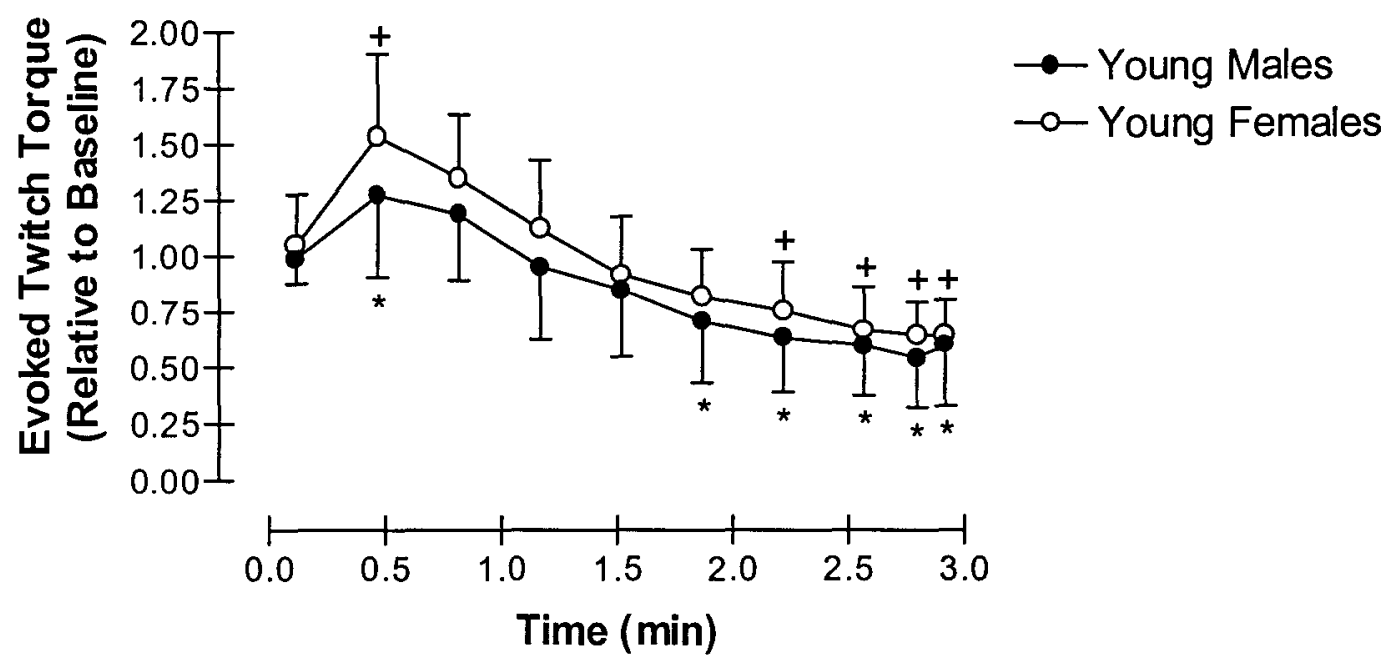

Figure 2b




Figure 3a

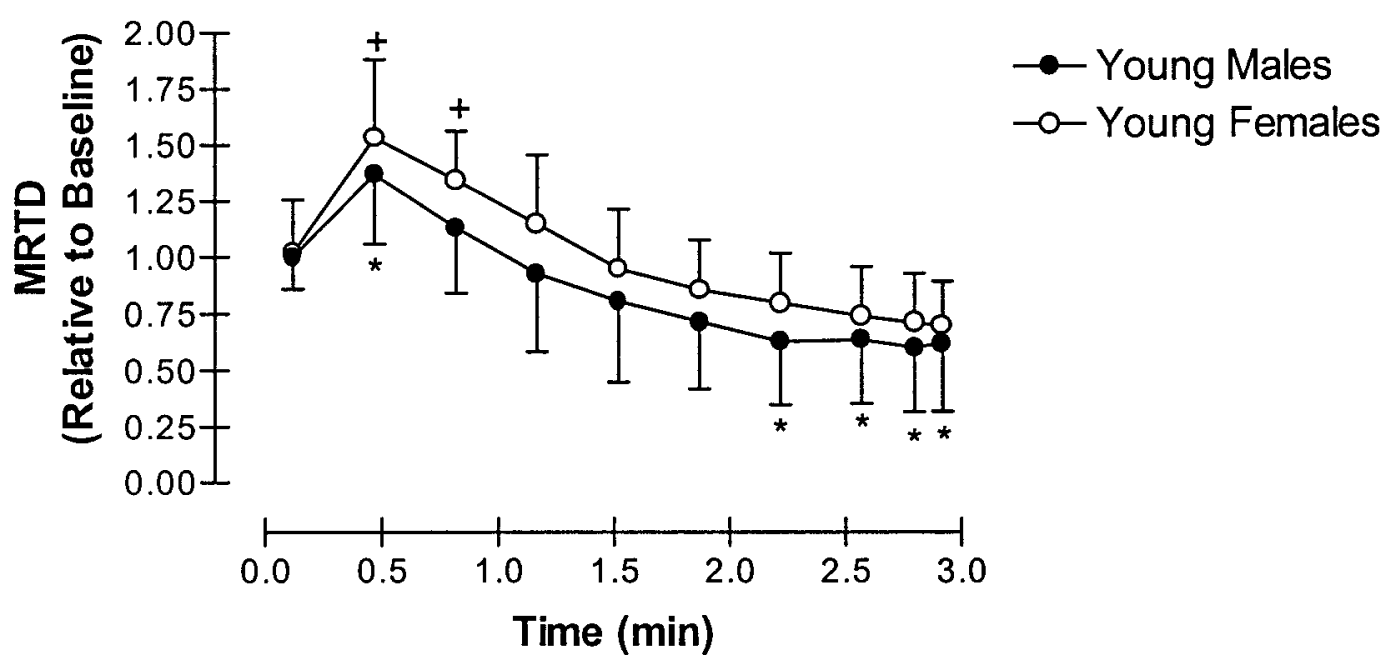

Figure $3 b$

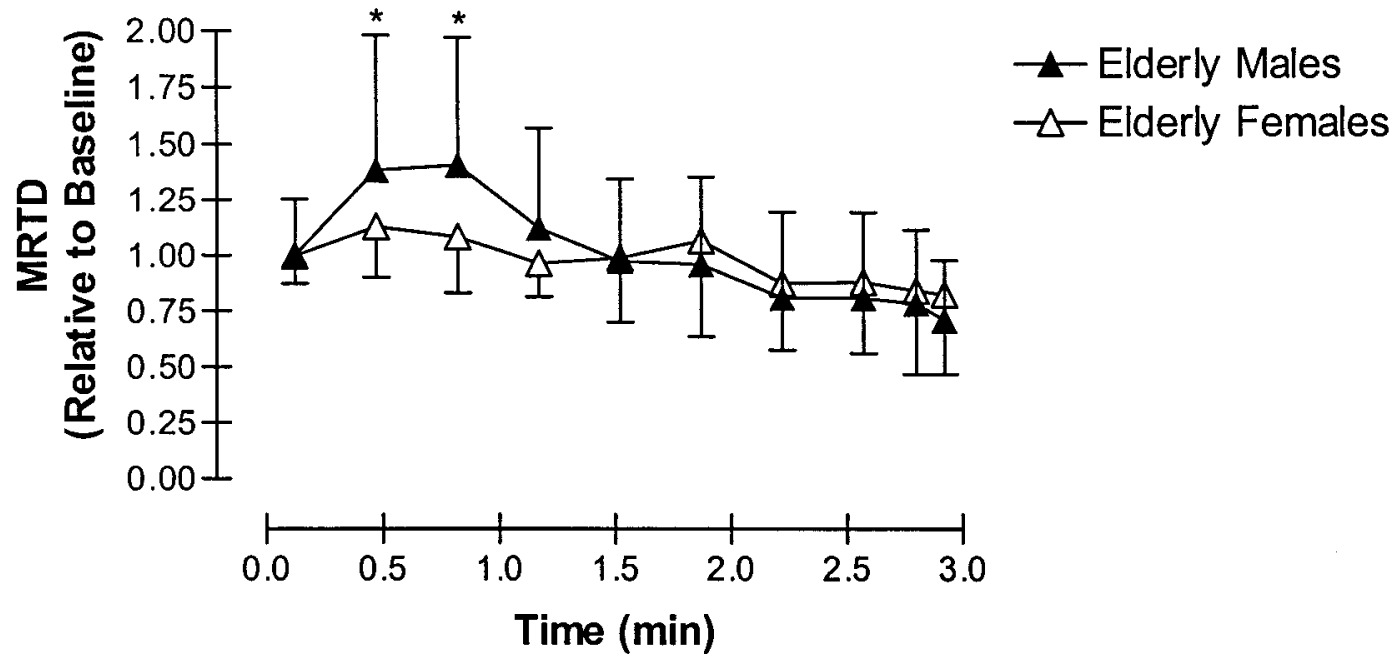


Figure 4

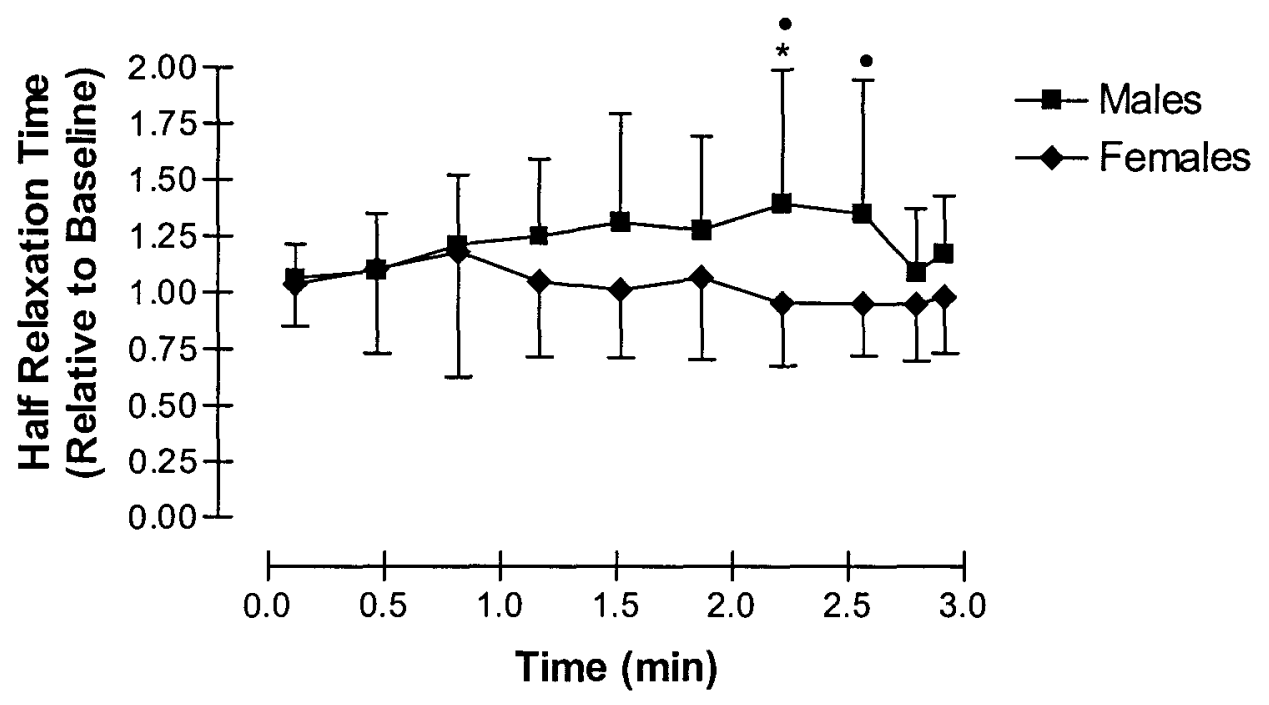


Figure 5a

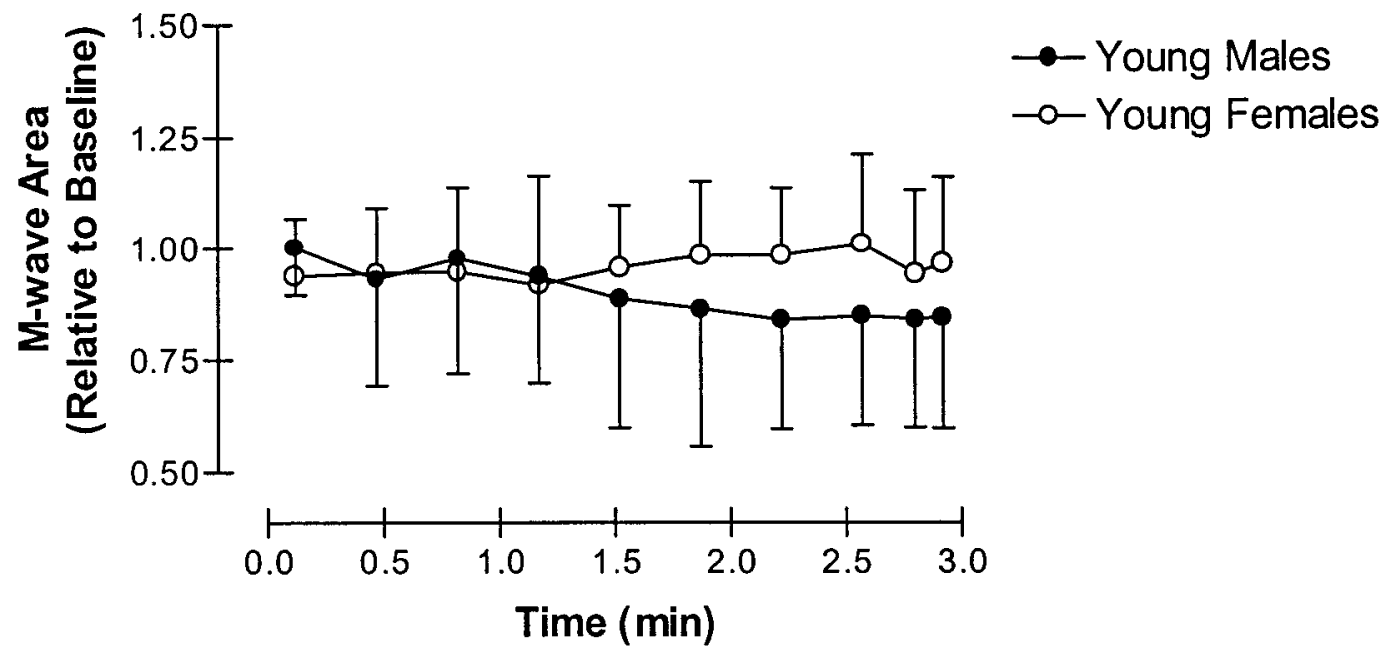

Figure 5b

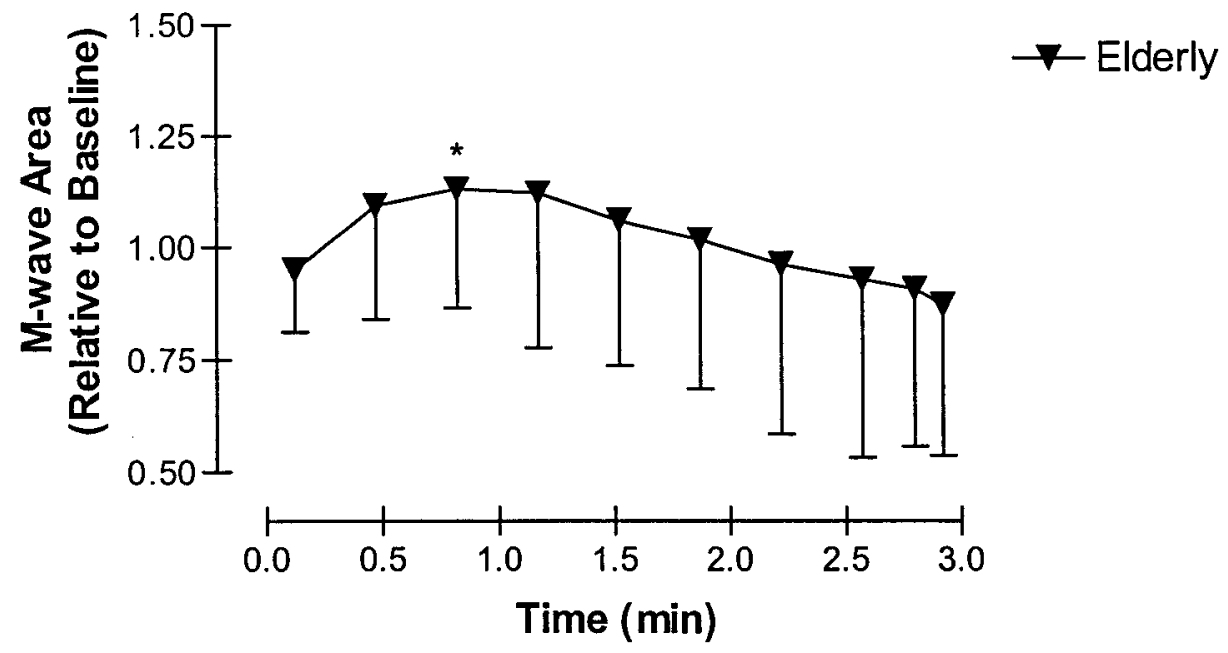


Figure $6 a$

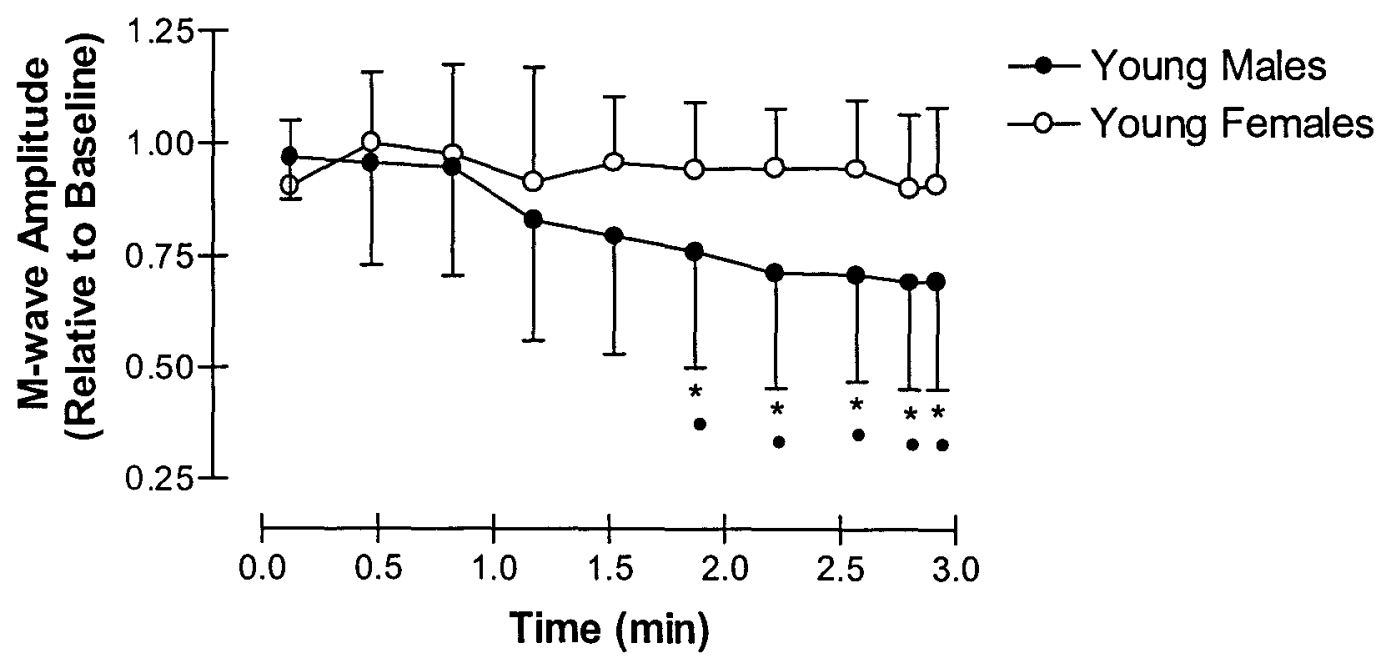

Figure $6 b$

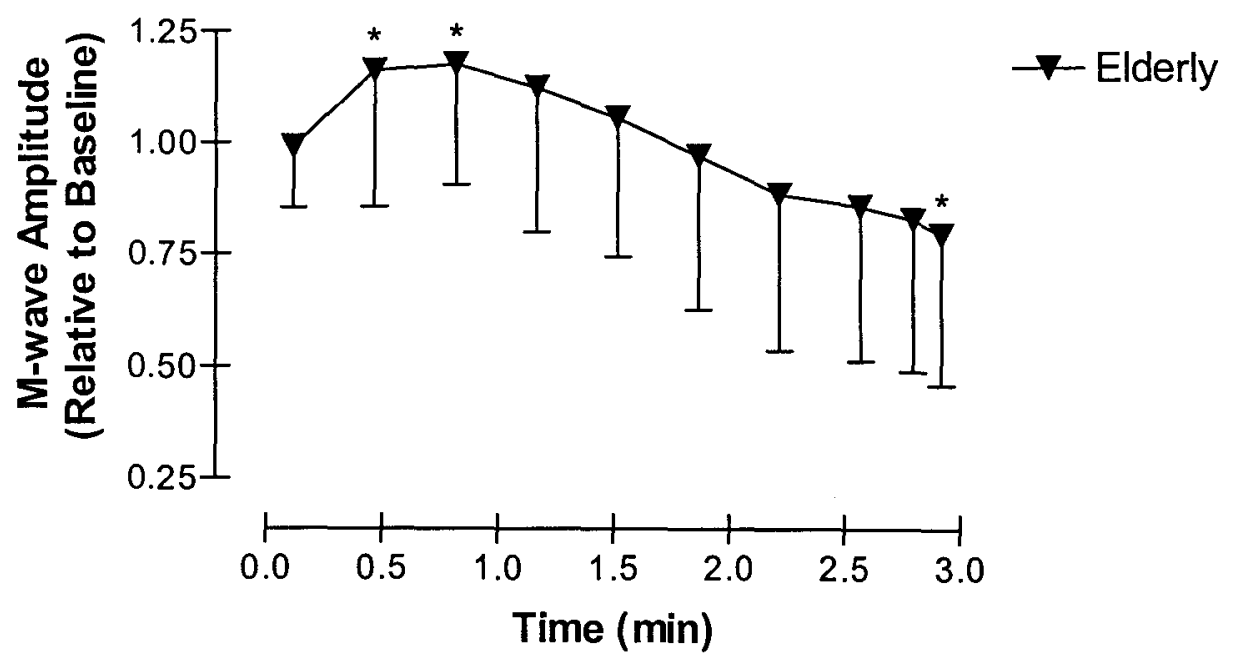


Figure 7

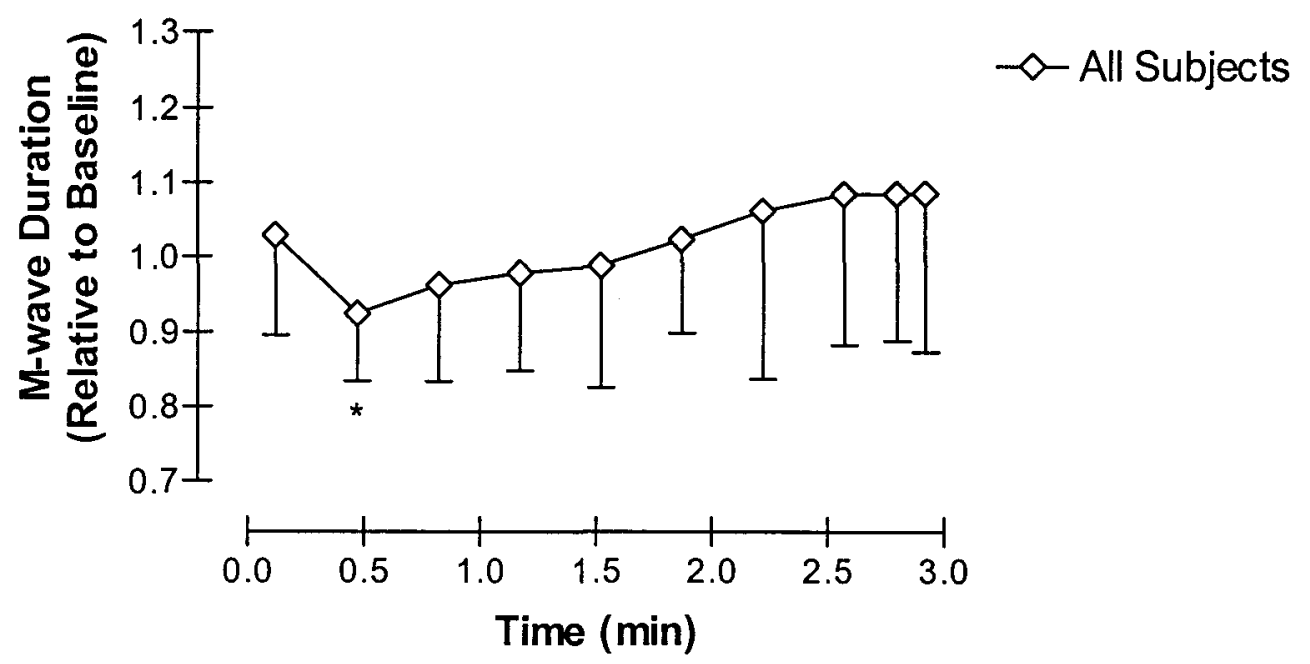


Figure 8a

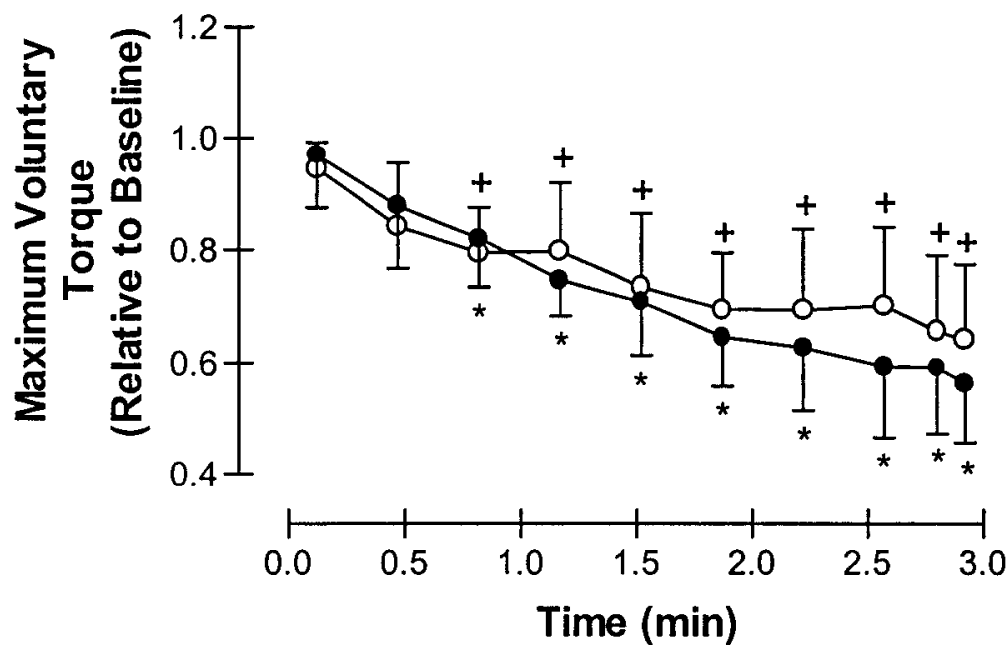

-- Young Males

- - Young Females

Figure 8b

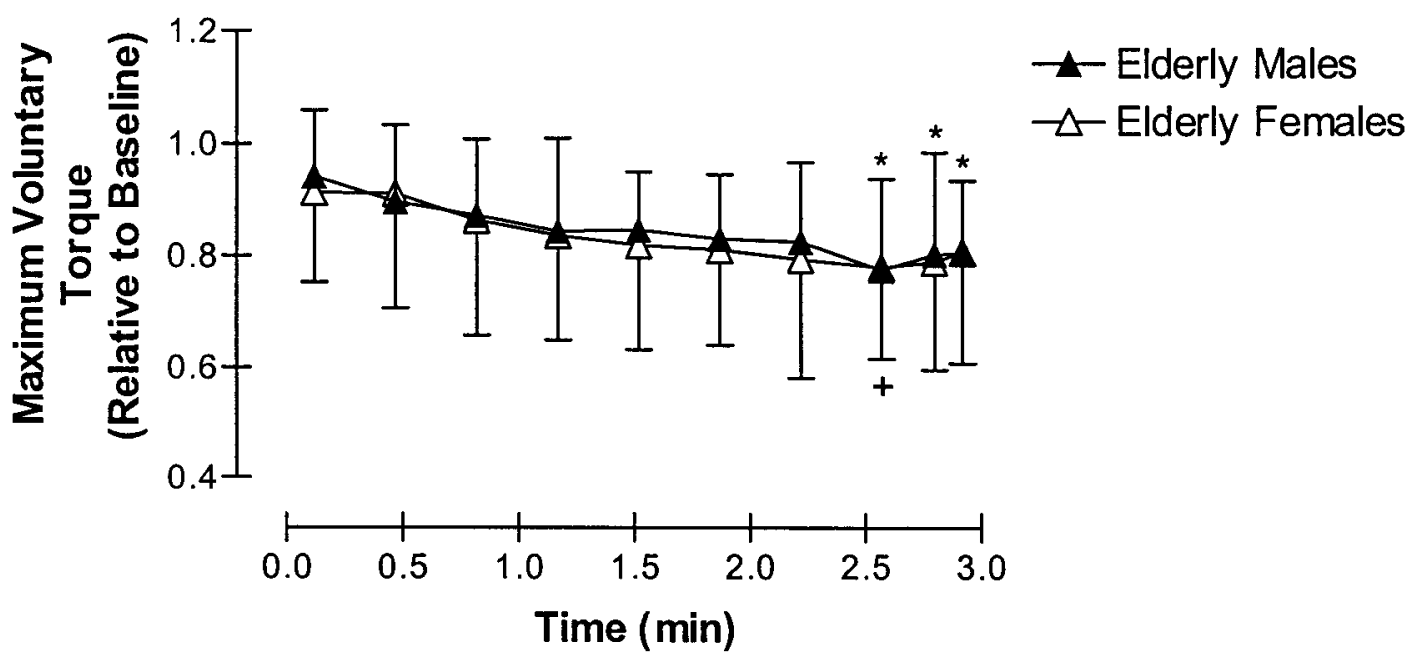


Figure 8c

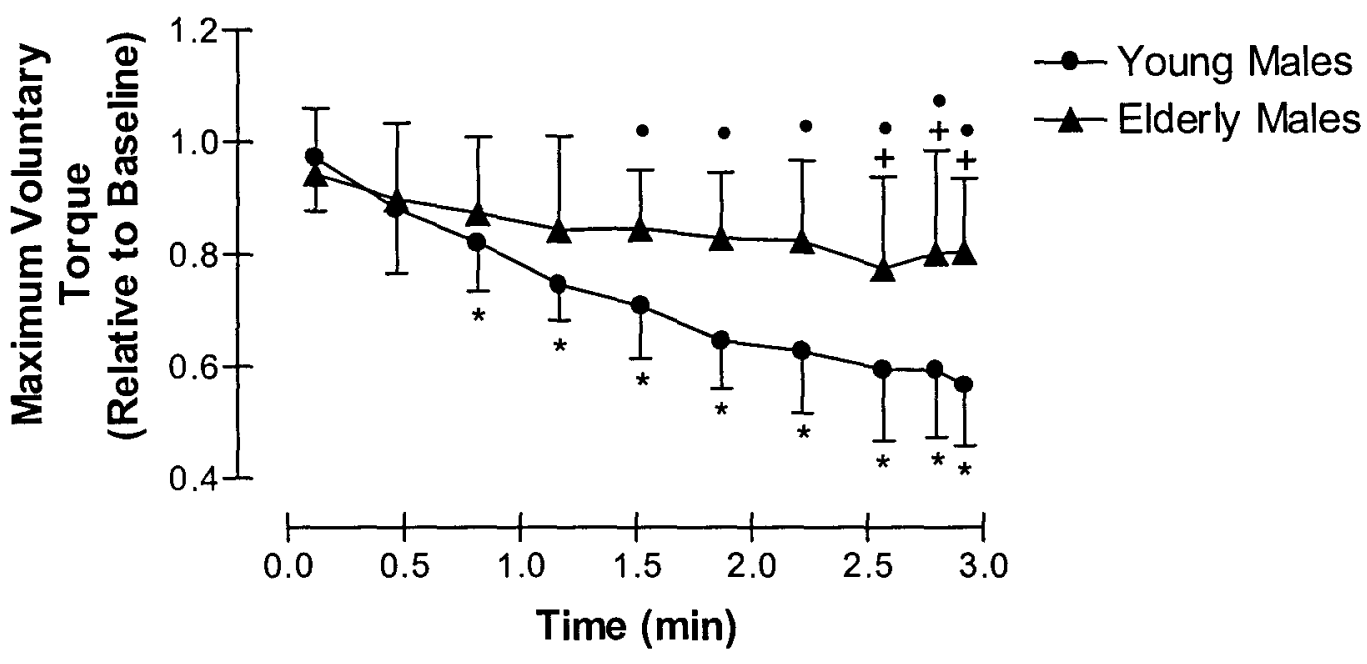

Figure 8d

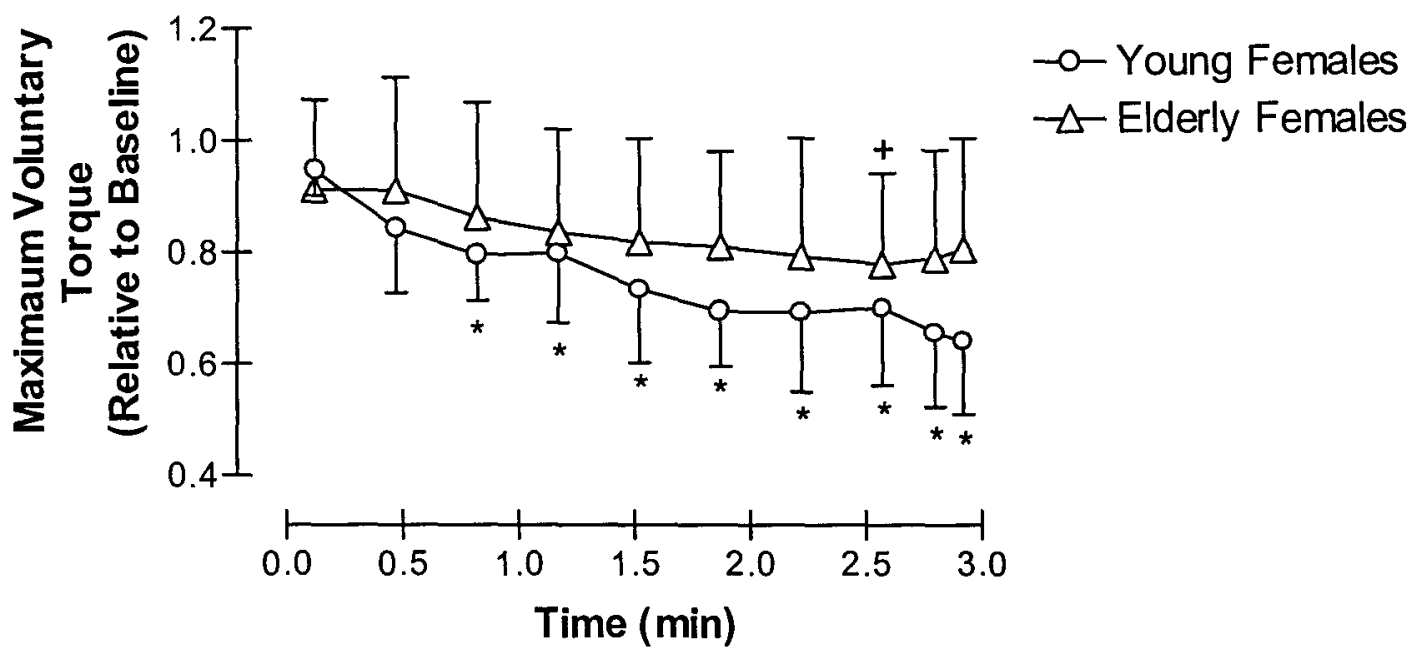


Figure 9

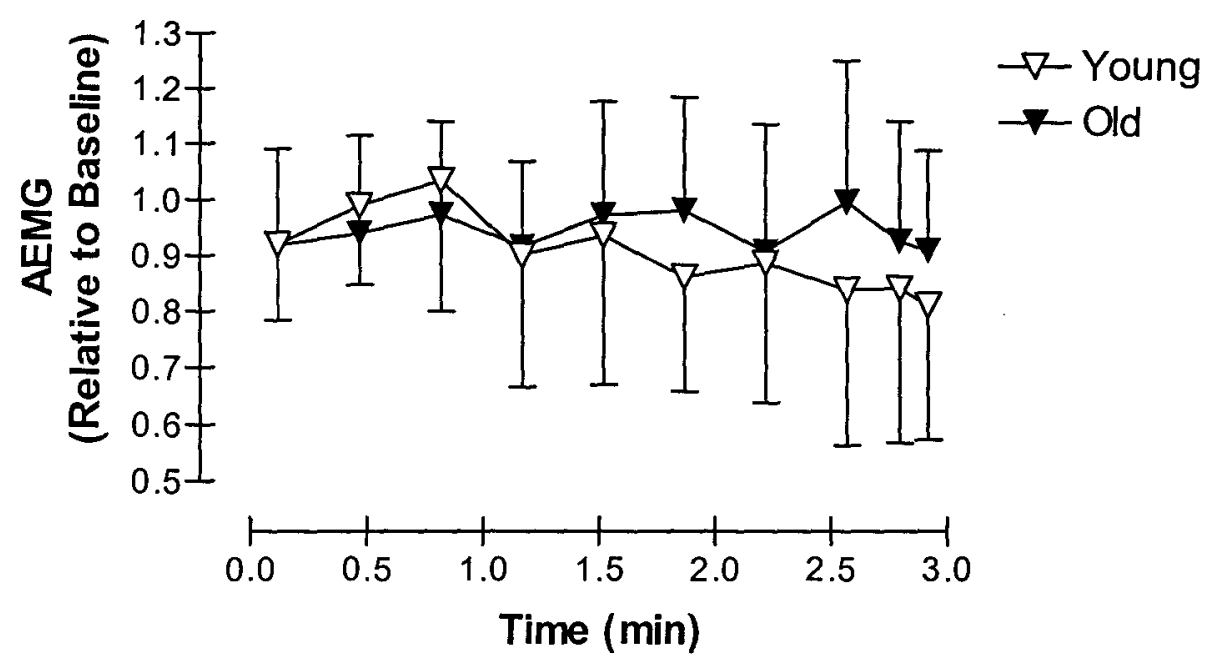


Figure 10

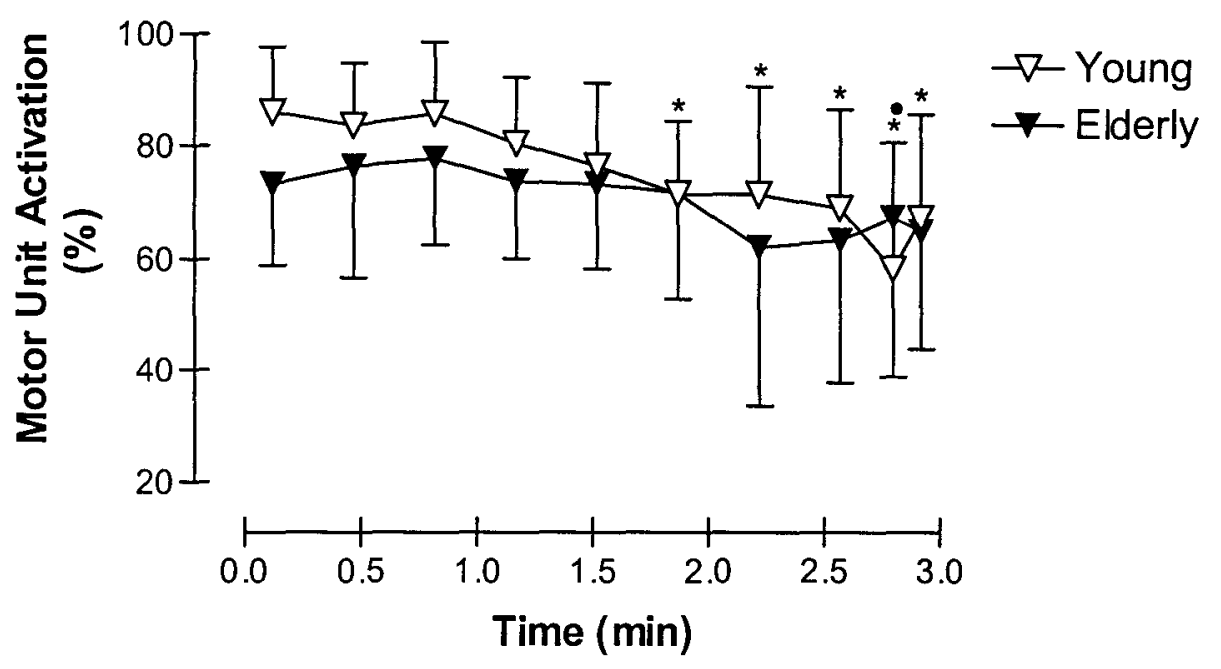


Figure 11

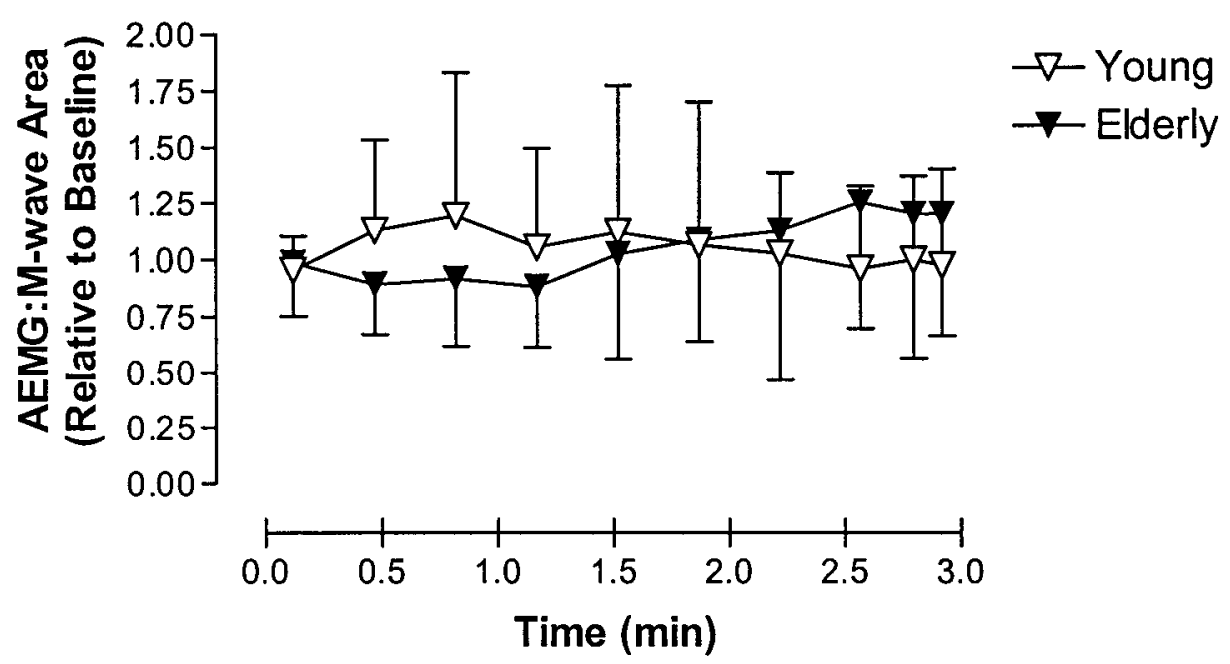




\section{Appendix A}

Subject Consent Form 


\author{
Department of Kinesiology \\ McMaster University \\ Hamilton, Ontario, Canada
}

Consent Form

\title{
FATIGUE IN THE HUMAN ADDUCTOR POLLICIS MUSCLE: THE EFFECTS OF AGE AND GENDER
}

I,

Dr. A. Hicks designed to examine fatigue characteristics in the thumb muscles of the hand in males and females. The results of this study will be made available to the scientific community, but I shall receive no monetary or other benefit from the study results or my participation.

I am aware that I will have electrodes taped to my skin to deliver an electrical stimulus to a nerve in my wrist and that I will have my hand strapped into a metal frame. I will be asked to either voluntarily contract my thumb against a resistance, or my muscle will be stimulated electrically. I have been told that there might be temporary discomfort associated with the stimulation, however, no short or long term side effects are expected from this procedure. I am aware that these identical procedures have been used in the past in dozens of subjects, with no reports of side-effects other than the temporary discomfort.

I am aware that I will be expected to come to the laboratory on one or two different occasions for the various tests that will be performed, and I understand that I may withdraw from the study at any time without repercussions, even after signing this form. Neither my name nor any reference to me will be used in compiling the results nor in publication in any form whatsoever.

I have had the study explained to me by either Dr. A. Hicks or Mr. Dave Ditor, and understand the nature of the investigation and my rights.

Name (print)

Witness (print)

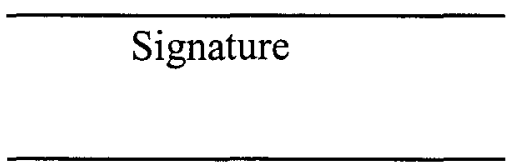

Signature
Date

Date

I (one of the investigators) have explained the nature of the study to the subject and believe that he/she understood it. 
Appendix B

Pilot Study 
The optimal joint angle for adductor pollicis force production in men and women. 


\begin{abstract}
The purpose of this study was i) to determine the joint angle that allows for the greatest MVC and evoked twitch forces from the adductor pollicis (AP), and ii) to determine if a gender difference exists in either the above forces or the optimum thumb angle. Ten males (25.2 yrs) and ten females (27.6 yrs) participated. The non-dominant hand was placed palmdown with the thumb fixed at four different angles of abduction $\left(55^{\circ}, 70^{\circ}, 85^{\circ}\right.$ and $\left.100^{\circ}\right)$. Male MVC forces were significantly greater than female $(9.2 \pm 1.8 \mathrm{~kg}$ vs. $6.4 \pm 1.4 \mathrm{~kg})$, and there was no significant effect of joint angle on MVC force in either gender. For the evoked twitch, men were significantly stronger than women when tested at the $100^{\circ}$ and $85^{\circ}$ angles $(0.82 \pm 0.23 \mathrm{~kg}$ vs. $0.58 \pm 0.11 \mathrm{~kg}$, and $0.82 \pm 0.20 \mathrm{~kg}$ vs. $0.59 \pm 0.11 \mathrm{~kg}$, respectively), and a significant effect was found for joint angle such that the lowest twitch force occurred at $55^{\circ}$. Males also tended to have a greater rate of force development than females $(p=0.07)$. These data suggest that studies utilizing the AP muscle in stimulated and voluntary paradigms should use a thumb angle between $70^{\circ}$ and $100^{\circ}$ of abduction, or approximately $85^{\circ}$, and that the same angle can be used for both males and females.
\end{abstract}

Key Words: evoked twitch, MVC, gender, EMG, rate of force development

\title{
Introduction:
}

A significant amount of research has made use of the adductor pollicis (AP) muscle 
in an attempt to clarify the mechanisms which account for skeletal muscle fatigue. The AP has proven ideal for the purposes of research as it is a relatively flat and superficial muscle, and even more importantly, because it is the only ulnar-supplied muscle acting on the thumb (Merton, 1954). Because of its unique innervation, one can measure stimulated responses of the $\mathrm{AP}$ and remain reasonably confident that such measurements are not contaminated by neighbouring muscles. However, a review of the existing literature shows that there is a great deal of variation in the hand position used for research with this muscle. Specifically, the angle formed between the thumb and the index finger $\left(\mathrm{A}_{\mathrm{TI}}\right)$ remains either unspecified in many experiments (Jones et al., 1979; Cooper et al., 1988) or inconsistent from one study to the next, varying between $50^{\circ}$ (Duchateau and Hainaut, 1985) and approximately $100^{\circ}$, i.e. the thumb fully abducted (Edwards et al., 1977).

As the AP spans between the third metacarpal and the base of the proximal phalanx of the thumb (Salmons, 1995), many of its characteristics may be determined by the degree of thumb abduction or $\mathrm{A}_{\mathrm{TI}}$. For example, the length of its sarcomeres and the tension in its elastic component are both influenced by thumb position, and therefore so too is its maximum force development and rate of force development. Since these latter two characteristics are often examined in studies of muscle fatigue, it follows that inter-study variability in $\mathrm{A}_{\mathrm{TI}}$ may significantly confound the interpretation of these studies and the fatigue mechanisms proposed. For the sake of inter-study reliability it seems warranted and necessary to determine a consistent, if not optimal, $A_{\mathrm{TI}}$ for research concerning fatigue which uses the AP muscle as a model. 
It is also possible that women, due to the greater series elastic component of their muscle (Bell and Jacobs, 1986; Winter and Brookes, 1991), may produce the optimal sarcomere length at a different $A_{T I}$ than men. The utilization of the same hand position when testing both genders may result in different sarcomere lengths between the groups, which in turn, may affect both voluntary and evoked force generation. While many studies have examined the gender difference in strength (Hicks and McCartney, 1996; West et al., 1995), or the effect of joint angle on the force generated by a muscle (Winegard et al., 1997), the literature remains scarce as to whether a gender difference exists in the optimal angle for muscular force production. It is therefore necessary to establish the optimal $A_{T I}$ for maximal AP force production and to determine whether or not there is a gender difference in this angle.

The purpose of the present study was therefore i) to determine the $A_{T I}$ that allows the greatest voluntary and evoked forces from the AP and ii) to determine whether or not a gender difference exists in either the MVC or evoked twitch forces or the optimal $\mathrm{A}_{\mathrm{TI}}$.

\section{Methods:}

Subjects:

20 normal healthy adults, 10 male (avg. age 25.2 yrs) and 10 females (avg. age 27.6 yrs), volunteered to participate in the study. None had any contraindication to electrical muscle stimulation or lacked the full range of motion of the thumb. The study was approved by the McMaster University Ethics Committee. 
Stimulating and recording:

Each subject placed his/her non-dominant hand palm-down in a custom designed apparatus. The four fingers and the wrist remained in a fixed position but the thumb could be manipulated and held at any degree of abduction. An adjustable chair faced the apparatus such that each subject could sit comfortably with his/her pronated forearm perpendicular to the plane of the body with the elbow flexed at approximately $135^{\circ}$.

After cleansing and lightly abrading the skin on both sides of the hand and wrist, a stimulating electrode (coated with conducting cream) was placed on the volar surface of the forearm, just proximal to the wrist and medial to the midline, with the cathode distal. In this position the electrode would lie directly over of the ulnar nerve. A Medi-trace pellet disposable electrode (Graphic Controls Canada Ltd., patent no. 1144606), consisting of a silver-silver chloride disc measuring $1 \mathrm{~cm}$ in diameter, was used as the recording electrode and was secured over the belly of the adductor pollicis muscle. A $25 \mathrm{~mm}$ x $5 \mathrm{~mm}$ silver strip was used as the reference electrode and was taped to the distal phalanx of the middle finger. Another Medi-trace disposable electrode was secured to the back of the wrist, between the recording and stimulating electrodes, to serve as a ground. A splint was put on the palmar side of the thumb which was then inserted into a strain gauge ring such that the ring straddled the distal joint. The strain gauge mount was magnetized to the table surface and could be moved to attain any desired thumb angle.

Evoked twitches:

To avoid any post-tetanic potentiation (Vandervoort et al., 1983; Houston et al., 
1985), all evoked twitches were performed prior to any maximal voluntary contractions (MVC).

Subjects began with the thumb abducted to $100^{\circ}$. A stimulator then delivered rectangular voltage pulses of $100 \mu$ s to evoke single twitches. The voltage was adjusted until there was no further increase in force; this was determined to be the peak twitch force (PT). This voltage was then used for all subsequent stimulations. Two twitches and corresponding compound muscle action potentials (M-waves) were then recorded at each of the following angles; $100^{\circ}, 85^{\circ}, 70^{\circ}$, and $55^{\circ}$. Measures were made for peak twitch force (PT), time to peak twitch force (TPT), half-relaxation time (HRT) and M-wave amplitude $\left(\mathrm{M}_{\text {amp }}\right)$. The rate of force development (RFD) was calculated by dividing the peak twitch force by the time taken for this peak force to be reached, and was expressed as $\mathrm{kg} / \mathrm{sec}$.

Maximum voluntary contractions:

Following all measurements of the evoked responses, subjects performed two maximal voluntary contractions at each of the above angles (starting at $55^{\circ}$ and ending at $100^{\circ}$ ). Verbal encouragement from the experimenter as well as visual feedback from the force monitoring screen were both provided in an attempt to fully motivate each subject to achieve their maximum voluntary force). A single twitch was interpolated on each 5 second MVC to determine the amount of motor unit activation, according to the method of Belanger and McComas (1981). A 0.5 second window of the electromyogram (EMG) associated with the MVC for each joint angle was analyzed for determination of voluntary EMG; average EMG (AEMG) was then determined by dividing the voluntary EMG by the $0.5 \mathrm{~s}$ time frame. 
EMG processing:

All EMG signals, corresponding to the M-wave and voluntary EMG data, were amplified using a Honeywell accudata EMG bio-amplifier at a sampling rate of $2.5 \mathrm{kHz}$ and a bandwidth of $15 \mathrm{~Hz}$ to $1.5 \mathrm{kHz}$. The Windac software program, ACODAS, (Dataq Instruments, Dayton, Ohio) was used to analyze all of the mechanical and M-wave recordings, and to full-wave digitally rectify and calculate the area under the curve for the voluntary EMG signals.

The M-wave:AEMG ratio was also calculated (M-wave amplitude (mV)/AEMG $(\mathrm{mV}))$. This value relates the evoked muscle membrane excitability to the voluntary activation of the muscle and was used to help determine potential mechanisms for any effect of joint angle on muscle activation.

Statistical analysis:

Each of the above dependent variables were analyzed with a two factor (gender $\mathrm{x}$ joint angle) between subjects analysis of variance (ANOVA) with repeated measures for joint angle. The Tukey A honestly significant difference post hoc test was used as needed to compare means. Statistical significance was established at $\mathrm{p}<.05$ and all data are presented as mean \pm standard deviation. 


\section{Results:}

\section{Table 1 here.}

The effect of gender and angle on evoked twitch properties:

Fig.1 shows the amplitude of the evoked response for both men and women at each of the four angles tested. There was a gender $\mathrm{x}$ joint angle interaction which showed that men were significantly stronger than women at the angles of $100^{\circ}$ and $85^{\circ}(0.82 \pm 0.23 \mathrm{~kg}$ vs. $0.58 \pm 0.11 \mathrm{~kg}$, and $0.82 \pm 0.20 \mathrm{~kg}$ vs. $0.59 \pm 0.11 \mathrm{~kg}$ respectively). There was also a main effect found for angle such that the lowest evoked twitches were elicited at $55^{\circ}$.

There was no difference between the genders for TPT, however there was a main effect for angle on TPT. The time required for peak twitch at $100^{\circ}$ was significantly greater than the time required at $55^{\circ}$ (Table 1). This effect of angle on TPT, however, is probably a result of the greater forces developed at the greater angles of stretch, i.e. more force was generated as the joint angle increased and these greater forces take more time to develop than the smaller forces produced at the less stretched angles.

There was no gender difference for HRT, although joint angle did have a significant effect on this variable (Table 1). The tendency for HRT to become shorter as the angle decreased was again partially due to the PT's becoming smaller and therefore requiring less time to relax.

The RFD showed a trend toward a gender difference $(p=0.07)$, such that men showed greater rates of force production than women, There was also a highly significant effect of joint angle on RFD ( $\mathrm{p}<0.001$ ), such that the $55^{\circ}$ angle produced lower rates of 
force production than the other three angles (Table 1).

Fig. 1 here.

The effect of gender and angle on MVC properties:

Table 2 here.

There was a significant main effect for gender on the MVC force generated by the adductor pollicis muscle $(\mathrm{p}<0.001)$. Men were found to be stronger than women $(9.2 \pm 1.8$ kg vs. $6.4 \pm 1.4 \mathrm{~kg}$ ) (Fig. 2). There was no effect for joint angle on the MVC force and there was no gender $\mathrm{x}$ joint angle interaction.

There was no effect of either gender or joint angle on the percent MUA during the maximal voluntary contractions (Table 2). The mean percent MUA was $86.2 \pm 13.8 \%$.

\section{Fig. 2 here.}

The effect of gender and angle on the $M$-wave and voluntary $E M G$ :

There was no gender difference found for the M-wave amplitude, however, there was a main effect for joint angle on M-wave amplitude such that the $100^{\circ}$ angle produced significantly greater values than the $55^{\circ}$ angle (Fig. 3).

Neither gender nor joint angle had a main effect on the voluntary EMG during the maximal voluntary contractions or the M-wave:AEMG ratio. The mean maximum voluntary EMG was $0.24 \pm 0.05 \mathrm{mVs}$ and the M-wave:AEMG ratio was $79.19 \pm 17.63$.

Fig. 3 here. 


\section{Discussion:}

A frequent oversight in studies concerning skeletal muscle fatigue is that no attempt is made to test subjects at a consistent sarcomere length. When there is variability in sarcomere length, subjects whose muscles are optimally stretched may produce greater maximal voluntary and evoked forces than others which are less stretched, but may also fatigue faster due to the greater energy requirement. The mechanisms behind muscle fatigue, as a result, may be further misunderstood. The problem becomes more severe in studies concerning the gender difference in muscle fatigue. Because females have a greater amount of series elastic component in their muscle (Bell and Jacobs, 1986; Winter and Brookes, 1991), their sarcomeres may be less stretched than a male's, despite a common joint position. When tested in this common position, which is often the case, females may not be producing their true maximal voluntary or evoked forces and therefore, may show an overestimated resistance to fatigue. The adductor pollicis is commonly used in fatigue research, but the variance in hand positions between studies suggests that there may be a problematic variance in sarcomere length. The purpose of the present study was to determine the optimal thumbindex finger angle $\left(\mathrm{A}_{\mathrm{TI}}\right)$ for force production in the AP muscle, and to determine whether or not a gender difference exists in either the forces produced or the optimal angle.

The effect of gender on the evoked twitch:

For the evoked twitch there was a significant gender $\mathrm{x}$ joint angle interaction which showed that while men were significantly stronger than women at $100^{\circ}$ and $85^{\circ}$ of thumb abduction, there was no gender difference at the $70^{\circ}$ or $55^{\circ}$ joint angles. These findings are 
very similar to those of Winegard et al. who found that the plantar flexors of college-aged men produced significantly greater evoked twitches than age-matched women (Winegard et al., 1997). Winegard also found a gender $\mathrm{x}$ joint angle interaction in the ankle plantar flexors such that men were stronger than women at the angles which produced the greatest muscle stretch $\left(20^{\circ}\right.$ dorsiflexion to $10^{\circ}$ plantarflexion), but not at the least stretched angle of $20^{\circ}$ plantarflexion. This gender difference in evoked twitch force has been shown previously in the dorsiflexors and elbow flexors, again with the muscle stretched at $20^{\circ}$ plantarflexion and $100^{\circ}$ elbow flexion, respectively (Hicks and McCartney, 1996).

It is likely that a large portion of the male strength advantage found in the present study was due to a similar male advantage in muscle mass (Sale et al., 1987; Schantz et al., 1983).

The present study showed a trend $(\mathrm{p}=.07)$ for men to have greater rates of evoked force production than women in the AP muscle. This finding is supported by the work of Bell and Jacobs in 1986 who found greater rates of force production in males as compared to females during MVC's of the biceps. It may be argued that women have slower rates of force development due to a greater 'elasticity' in their muscles (Bell and Jacobs, 1986; Winter and Brookes, 1991), however, it is noteworthy that the greater rates of force development observed in males in the present study were most obvious at the angles of greatest stretch.

The effect of joint angle on the evoked twitch:

There was a main effect for angle on the evoked twitch such that the forces produced during the $55^{\circ}$ angle (the least stretched condition) were significantly lower than the forces 
produced during each of the other angles. This length-tension relationship has been commonly observed in many different muscles (Sale et al., 1982; Marsh et al., 1981). Increasing the $\mathrm{A}_{\mathrm{TI}}$ in the present study may have produced sarcomere lengths more conducive to force production, however, this is probably not the only explanation. The higher twitch forces produced at the larger angles may be due to the stretching of the series elastic component of the muscle. The fact that the $55^{\circ}$ angle not only produced smaller forces, but also significantly slower rates of force production supports this hypothesis. However, it has been suggested that females have a greater series elastic component to their muscle than males (Bell and Jacobs, 1986; Winter and Brookes, 1991). If the series elastic component was in fact responsible for the angle effect, then females would be expected to show greater relative strength increases in peak twitch force as their muscles are stretched as compared to males. However, this was not the case; the strength increase from the $55^{\circ}$ angle to the $100^{\circ}$ angle was less obvious in females as compared to males (Fig. 1), which weakens the hypothesis that the series elastic component is primarily responsible for the angle effect on PT.

Previous studies have examined $\mathrm{M}$-wave data to determine if muscle membrane excitability increases as the muscle is stretched, and the results are somewhat conflicting (Sale et al., 1982; Fitch and McComas, 1985). In the present study, although the M-wave amplitude was significantly larger at the $100^{\circ}$ angle as compared to the $55^{\circ}$ angle, the Mwave:AEMG ratio was not affected by the $A_{\mathrm{TI}}$. This suggests that the increase in M-wave amplitude at the greater angles was not likely due to any specific change in muscle 
membrane excitability, but rather, the stretching of the skin under the recording electrode resulted in a proportional increase in both the evoked and voluntary electromyographical signals.

The effect of gender on the MVC:

The present study found a distinct effect for gender on the MVC force generated by the AP, such that males were stronger than females. This gender difference in strength has been frequently observed over a wide range of muscles (West et al., 1995; Bell and Jacobs, 1986), and seems to persist despite the effects of aging (Hicks and McCartney, 1996) and when expressed relative to body mass (Bell and Jacobs, 1986). The gender difference in muscle size may not be the sole contributor to the gender difference in strength. The question of a gender difference in the specific tension (strength/CSA) of muscle has yet to be adequately answered. One study, which examined the quadriceps, reported males to be approximately $25 \%$ stronger than women per unit of muscle cross section (Kanehisa et al., 1994). Another study, however, examined the adductor pollicis and found no gender difference in the specific tension of this muscle in pre-menopausal women and age-matched men (Phillips et al., 1993).

The fact that no gender difference was observed in either motor unit activation or Mwave characteristics excludes the possibility that central drive and/or muscle membrane excitability were contributing factors to the gender difference in strength.

The effect of angle on the MVC:

In contrast to the evoked twitch, there was no significant effect found for joint angle 
on the MVC force. It is unlikely that central activation factors could account for the absence of any significant joint angle effect since the estimates of motor unit activation were not different between joint angles, nor were there any significant differences in voluntary EMG.

The most likely explanation for the lack of a joint angle effect is that other muscles may have been recruited during the voluntary thumb adduction, which may mask the specific influence of the AP. Despite the palm down position of the hand which was meant to discourage thumb flexion or opposition, it is impossible to eliminate the innervation of the median-supplied muscles during this voluntary contraction protocol. These additional muscles of the hand may have confounded the specific length-tension relationship of the AP during voluntary effort.

\section{Conclusion:}

Taking together both the voluntary and evoked data, it would appear that the optimal angle for force production in the AP muscle lies between $70^{\circ}$ and $100^{\circ}$, or approximately $85^{\circ}$. It also seems that there is no gender difference in this optimal angle. These results can now be used as guidelines for future studies utilizing the AP muscle as a model for muscle fatigue. 


\section{References}

Belanger, A.Y. and McComas, A.J. (1981). Extent of motor unit activation during effort. J. Appl. Physiol. 51: 1131-1135.

Bell, D.G. and Jacobs, I. (1986). Electro-mechanical response times and rate of force development in males and females. Med. Sci. Sports Exerc. 18:31-36.

Cooper, R.G., Edwards, R.H.T., Gibson, H. and Stokes, M.J. (1988). Human muscle fatigue: Frequency dependence of excitation and force generation. J. Physiol. 397: 585-599.

Duchateau, J. and Hainaut, K. (1985). Electrical and mechanical failures during sustained and intermittent contractions in humans. J. Appl. Physiol. 58(3): 942-947.

Edwards, R.H.T., Hill, D.K., Jones, D.A. and Merton, P.A. (1977). Fatigue of long duration in human skeletal muscle after exercise. J. Physiol. 272: 769-778.

Fitch, S. and McComas, A.J. (1985). Influence of human muscle length on fatigue. J. Physiol. 362: 205-213.

Hicks, A. and McCartney, N. (1996). Gender differences in isometric contractile properties and fatigability in elderly human muscle. Can. J. Appl. Physiol. 21(6): 441-454.

Houston, M.E., Green, H.J. and Stull, J.T. (1985). Myosin light chain phosphorylation and isometric twitch potentiation in intact human muscle. Pflugers Arch. 403: 348-352.

Jones, D.A., Bigland-Ritchie, B. and Edwards, R.H.T. (1979). Excitation frequency and muscle fatigue: Mechanical responses during voluntary and stimulated contractions. Exp. Neurol. 64: 401-413. 
Kanehisa, H., Ikegawa, S. and Fukunaga, T. (1994). Comparison of muscle cross-sectional area and strength between untrained women and men. Eur. J. Appl. Physiol. 68: $148-154$.

Marsh, E., Sale, D., McComas, A.J. and Quinlan, J. (1981). Influence of joint position on ankle dorsiflexion in humans. J. Appl. Physiol. 51(1): 160-167.

Merton, P.A. (1954). Voluntary strength and fatigue. J. Physiol. 123:553-564.

Phillips, S.K., Rook, K.M., Siddle, N.C., Bruce, S.A. and Woledge, R.C. (1993). Muscle weakness in women occurs at an earlier age than in men, but strength is preserved by hormone replacement therapy. Clin. Sci. 84:95-98.

Sale, D., MacDougall, J.D., Alway, S.E. and Sutton, J.R. (1987). Voluntary strength and muscle contractions in untrained men and women and male bodybuilders. J. Appl. Physiol. 62: 1786-1793.

Sale, D., Quinlan, J., Marsh E., McComas, A.J. and Belanger, A.Y. (1982). Influence of joint position on ankle plantarflexion in humans. J. Appl. Physiol. 52(6): 1636-1642.

Salmons, S. (Ed.). (1995). Muscle. In: Gray's Anatomy ( $38^{\text {th }}$ ed.), pg. 859. New York: Churchill Livingstone.

Schantz, P., Randall-Fox E., Hutchinson, W., Tyden, A. and Astrand, P.O. (1983). Muscle fibre type distribution, muscle cross-sectional area and maximal voluntary strength in humans. Acta Physiol. Scand. 117: 219-226.

Vandervoort, A.A., Quinlan, J. and McComas, A.J. (1983). Twitch potentiation after voluntary contraction. Exp. Neurol. 81: 141-152. 
West, W., Hicks, A., Clements, L. and Dowling, J. (1995). The relationship between voluntary electromyogram, endurance time and intensity of effort in isometric handgrip exercise. Eur. J. Appl. Physiol. 71: 301-305.

Winegard, K.J., Hicks, A.L. and Vandervoort A.A. (1997). An evaluation of the lengthtension relationship in elderly human plantarflexor muscles. J. Gerontol: Biological sciences. $52 \mathrm{~A}(6)$ : B337-B343.

Winter, E.M. and Brookes, F.B.C. (1991). Electromechanical response times and muscle elasticity in men and women. Eur. J. Appl. Physiol. 63: 124-128. 


\section{Acknowledgments}

We would like to extend a very special thanks to John Moroz for his technical support. 


\section{Figure Captions}

Figure 1. The effect of joint angle and gender on the evoked twitch force. Data points represent means $\pm \mathrm{SD}$. ( $\mathrm{n}=10$ for each group).

$\star$ Significantly different from female $(\mathrm{p}<.05)$.

+ Significantly different from all other joint angles $(\mathrm{p}<.05)$.

Figure 2. The effect of joint angle and gender on the MVC force. Data points represent means \pm SD. ( $\mathrm{n}=10$ for each group).

$\star$ MVC in males significantly greater than in females $(p<.05)$.

Figure 3. The effect of joint angle and gender on the M-wave amplitude. Data points represent means $\pm \mathrm{SD}$. ( $\mathrm{n}=10$ for each group).

+ Significantly different from the $100^{\circ}$ joint angle $(p<.05)$ 
Fig. 1

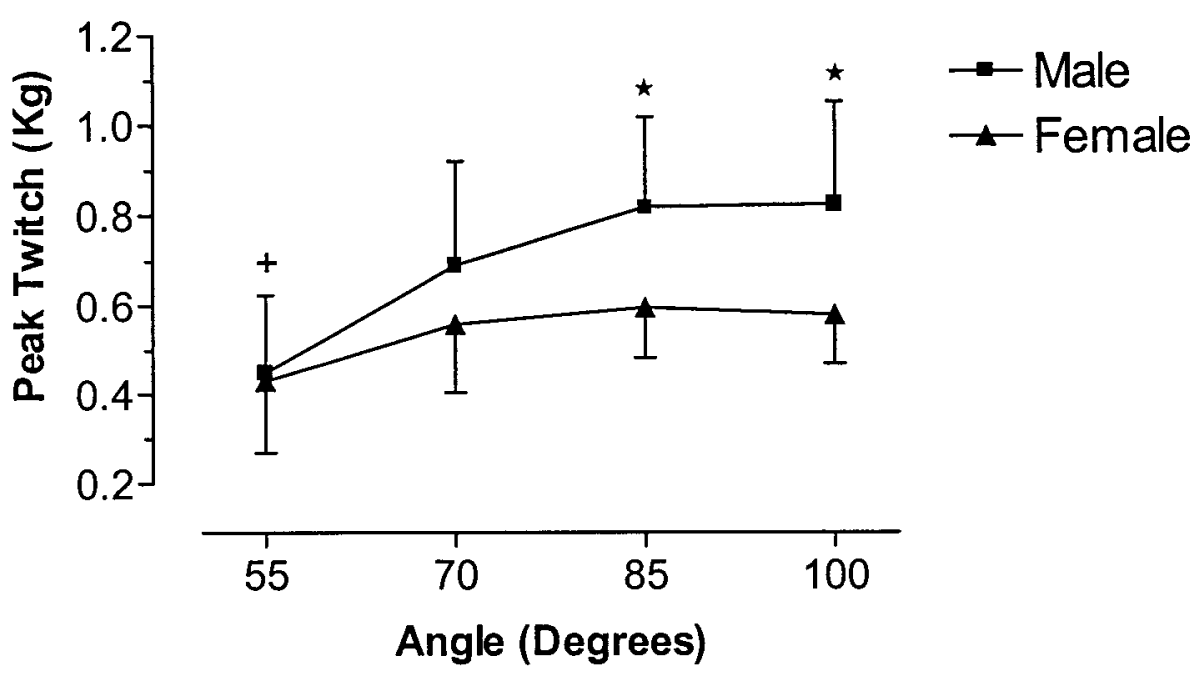


Fig. 2

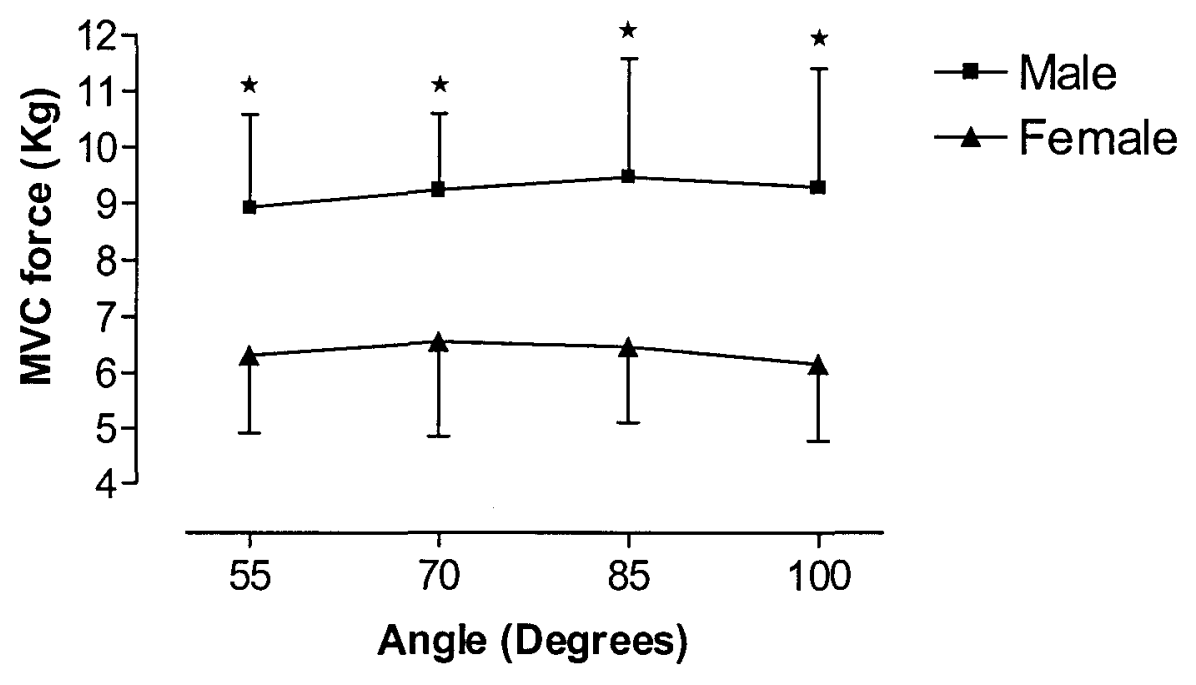


Fig. 3

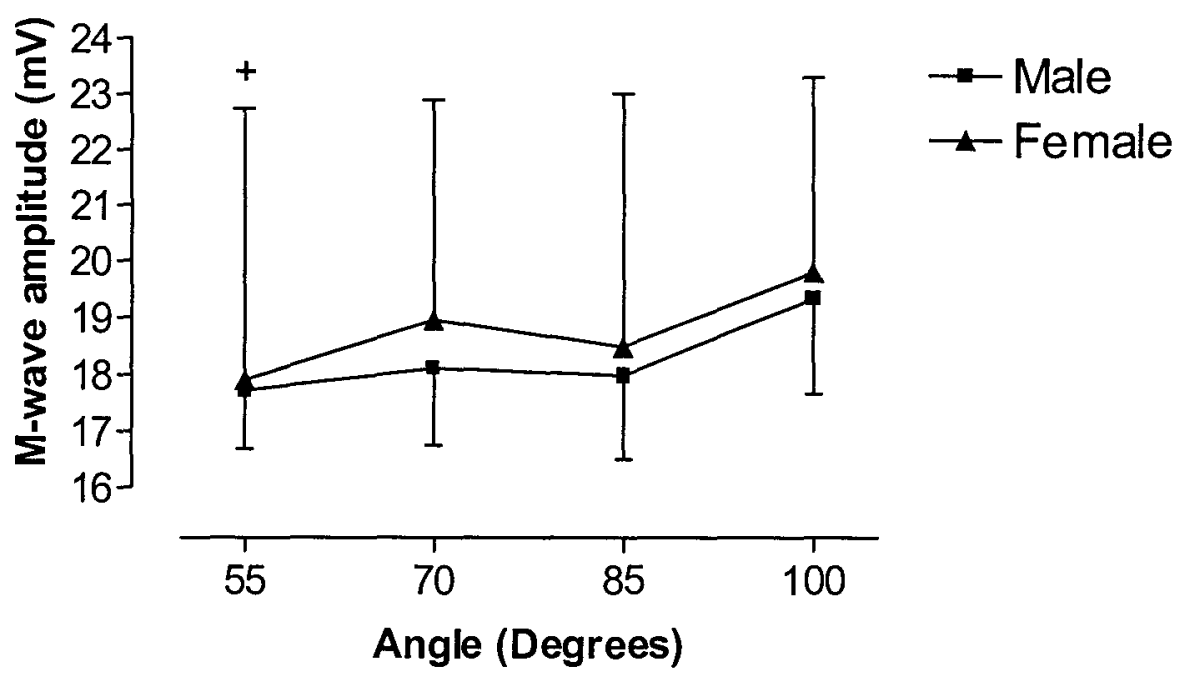


Table 1. Time to Peak Twitch (TPT), Half Relaxation Time (HRT) and Rate of Evoked Force Development (RFD) at Each Angle of Thumb Abduction.

\begin{tabular}{cccccc}
\hline \multicolumn{5}{c}{ ANGLE (DEGREES OF ABDUCTION) } \\
\hline $\begin{array}{c}\text { TPT } \\
(\mathrm{msec})\end{array}$ & Male & $\mathbf{1 0 0}$ & $\mathbf{7 5}$ & $\mathbf{7 0}$ & $\mathbf{5 5}$ \\
& Female & $70.7 \pm 11.9$ & $69.3 \pm 14.0$ & $68.5 \pm 13.5$ & $70.7 \pm 17.4$ \\
HRT & Male & $86.3 \pm 9.4$ & $78.7 \pm 8.9$ & $70.3 \pm 6.6$ & $58.8 \pm 6.0$ \\
$(\mathrm{msec})$ & Female & $86.7 \pm 26.8$ & $83.2 \pm 18.5$ & $69.0 \pm 13.7$ & $62.3 \pm 11.4$ \\
& Male & $11.2 \pm 2.9$ & $11.4 \pm 2.7$ & $10.4 \pm 3.2$ & $7.2 \pm 2.8$ \\
$\begin{array}{c}\text { RFD } \\
(\mathrm{Kg} / \mathrm{s})\end{array}$ & Female & $8.5 \pm 2.6$ & $8.9 \pm 2.8$ & $8.4 \pm 3.0$ & $6.3 \pm 2.7$ \\
\hline
\end{tabular}

Values are means \pm SD.

Table 2. Percent Motor Unit Activation (\%MUA) at Each Angle of Thumb Abduction.

\begin{tabular}{lccccc}
\hline \multicolumn{5}{c}{ ANGLE } & (DEGREES OF ABDUCTION) \\
\hline & \multicolumn{100}{c}{} & $\mathbf{8 5}$ & $\mathbf{7 0}$ & $\mathbf{5 5}$ \\
\hline \%MUA & Male & $83.3 \pm 17.6$ & $88.1 \pm 12.3$ & $91.7 \pm 8.8$ & $94.7 \pm 7.4$ \\
& Female & $82.4 \pm 9.4$ & $82.2 \pm 15.5$ & $86.2 \pm 17.1$ & $83.3 \pm 15.5$ \\
\hline
\end{tabular}

Values are means \pm SD. 
Appendix C

ANOVA Tables 


\begin{tabular}{cccccrr}
\hline \multicolumn{7}{c}{ Baseline Peak Torque - All Subjects } \\
\hline Effect & df & MS & df & MS & 2 = Gender \\
& Effect & Effect & Error & Error & F & p-level \\
\hline $\mathbf{1}$ & 1 & 0.76457 & 44 & 0.029845 & 25.6181 & $0.000008^{\star}$ \\
$\mathbf{2}$ & 1 & 0.855468 & 44 & 0.029845 & 28.66377 & $0.000003^{*}$ \\
$\mathbf{1 \times 2}$ & 1 & 0.002324 & 44 & 0.029845 & 0.07787 & 0.78151 \\
\hline
\end{tabular}

\begin{tabular}{|c|c|c|c|c|c|c|}
\hline \multicolumn{7}{|c|}{$\begin{array}{l}\text { Baseline Peak Torque (Controlled for Moment Arm Length) - All Subjects } \\
\qquad 1=\text { Age } 2=\text { Gender }\end{array}$} \\
\hline Effect & $\begin{array}{c}\text { df } \\
\text { Effect }\end{array}$ & $\begin{array}{c}\text { MS } \\
\text { Effect }\end{array}$ & $\begin{array}{c}\text { df } \\
\text { Error }\end{array}$ & $\begin{array}{c}\text { MS } \\
\text { Error }\end{array}$ & $\bar{F}$ & p-level \\
\hline 1 & 1 & 448.6943 & 44 & 35.01302 & 12.81507 & $0.000853^{\star}$ \\
\hline 2 & 1 & 679.9948 & 44 & 35.01302 & 19.4212 & $0.000066^{\star}$ \\
\hline $1 \times 2$ & 1 & 58.6775 & 44 & 35.01302 & 1.67588 & 0.202226 \\
\hline
\end{tabular}

\begin{tabular}{|c|c|c|c|c|c|c|}
\hline \multicolumn{7}{|c|}{$\begin{array}{l}\text { Baseline Maximum Rate of Evoked Torque Development - All Subjects } \\
\qquad \begin{array}{l}1=\text { Age } \quad 2=\text { Gender } \\
\end{array}\end{array}$} \\
\hline Effect & $\begin{array}{c}\text { df } \\
\text { Effect }\end{array}$ & $\begin{array}{c}\text { MS } \\
\text { Effect }\end{array}$ & $\begin{array}{c}\mathrm{df} \\
\text { Error }\end{array}$ & $\begin{array}{c}\text { MS } \\
\text { Error }\end{array}$ & $\mathbf{F}$ & p-level \\
\hline 1 & 1 & 398.8803 & 44 & 21.53496 & 18.52246 & $0.000092^{\star}$ \\
\hline 2 & 1 & 663.425 & 44 & 21.53496 & 30.80688 & $0.000002^{*}$ \\
\hline $1 \times 2$ & 1 & 1.4043 & 44 & 21.53496 & 0.06521 & 0.799636 \\
\hline
\end{tabular}

\begin{tabular}{ccccccr}
\hline \multicolumn{7}{c}{ Baseline Half Relaxation Time - All Subjects } \\
1 = Age & $\mathbf{2}=$ Gender \\
\hline Effect & df & MS & df & MS & F & p-level \\
& Effect & Effect & Error & Error & 0.913228 \\
\hline 1 & 1 & 0.000002 & 44 & 0.000199 & 0.012011 & $0.018562^{*}$ \\
$\mathbf{2}$ & 1 & 0.001187 & 44 & 0.000199 & 5.97767 & 0.464134 \\
\hline 1x2 & 1 & 0.000108 & 44 & 0.000199 & 0.545378 & \\
\hline
\end{tabular}

\begin{tabular}{cccccrr}
\hline \multicolumn{7}{c}{ Baseline M-wave Area - All Subjects } \\
1 = Age & $\mathbf{2}=$ Gender \\
\hline Effect & df & MS & df & MS & F & p-level \\
& Effect & Effect & Error & Error & \\
\hline $\mathbf{1}$ & 1 & 0.006664 & 33 & 0.000246 & 27.04994 & $0.00001^{*}$ \\
$\mathbf{2}$ & 1 & 0.000154 & 33 & 0.000246 & 0.62538 & 0.434703 \\
$\mathbf{1 \times 2}$ & 1 & 0.000009 & 33 & 0.000246 & 0.03581 & 0.851075 \\
\hline
\end{tabular}




\begin{tabular}{ccccccc}
\hline \multicolumn{7}{c}{ Baseline M-wave Amplitude - All Subjects } \\
1 = Age & $\mathbf{2}=$ Gender \\
\hline Effect & df & MS & df & MS & F & \\
& Effect & Effect & Error & Error & \\
\hline $\mathbf{1}$ & 1 & 444.3136 & 33 & 11.33007 & 39.21545 & $.000000^{*}$ \\
$\mathbf{2}$ & 1 & 6.8863 & 33 & 11.33007 & 0.60779 & 0.441178 \\
$\mathbf{1 \times 2}$ & 1 & 0.7234 & 33 & 11.33007 & 0.06385 & 0.802076 \\
\hline
\end{tabular}

\begin{tabular}{ccccccc}
\hline \multicolumn{7}{c}{ Baseline M-wave Duration - All Subjects } \\
1 = Age & 2 = Gender & p-level \\
\hline Effect & df & MS & df & MS & F & \\
& Effect & Effect & Error & Error & 0.396913 \\
& 1 & 0.000011 & 33 & 0.000014 & 0.736695 & 0.102055 \\
$\mathbf{1}$ & 1 & 0.00004 & 33 & 0.000014 & 2.828347 & 0.863838 \\
\hline $\mathbf{1 x 2}$ & 1 & 0 & 33 & 0.000014 & 0.029871 & \\
\hline
\end{tabular}

\begin{tabular}{ccccccc}
\hline \multicolumn{7}{c}{ Baseline MVC - All Subjects } \\
1 = Age & 2 = Gender \\
\hline Effect & df & MS & df & MS & F & p-level \\
& Effect & Effect & Error & Error & \\
\hline 1 & 1 & 48.4812 & 44 & 3.091709 & 15.68104 & $0.00027^{*}$ \\
2 & 1 & 62.42641 & 44 & 3.091709 & 20.19156 & $0.00005^{*}$ \\
$1 \times 2$ & 1 & 16.73241 & 44 & 3.091709 & 5.41203 & $0.024668^{*}$ \\
\hline
\end{tabular}

\section{Baseline MVC (Controlled for Moment Arm Length) - All Subjects}

\begin{tabular}{ccccccc}
\multicolumn{8}{c}{$\begin{array}{c}\text { 1 = Age } \\
\text { Effect }\end{array}$} & $\begin{array}{c}\text { df } \\
\text { Effect }\end{array}$ & $\begin{array}{c}\text { MS } \\
\text { Effect }\end{array}$ & $\begin{array}{c}\text { df } \\
\text { Error }\end{array}$ & $\begin{array}{c}\text { MS } \\
\text { Error }\end{array}$ & p-level \\
& 1 & 24178.69 & 44 & 3246.24 & 7.44821 & $0.009094^{*}$ \\
\hline $\mathbf{1}$ & 1 & 43333.71 & 44 & 3246.24 & 13.34889 & $.000685^{\star}$ \\
$\mathbf{1 \times 2}$ & 1 & 42031.2 & 44 & 3246.24 & 12.94765 & $.000807^{*}$ \\
\hline
\end{tabular}

\begin{tabular}{ccccccc}
\hline \multicolumn{7}{c}{ Baseline AEMG - All Subjects } \\
1 = Age & $\mathbf{2}=$ Gender \\
\hline Effect & df & MS & df & MS & F & p-level \\
& Effect & Effect & Error & Error & 0.11119 \\
\hline $\mathbf{1}$ & 1 & 0.101356 & 33 & 0.037835 & 2.678877 & 0.236567 \\
$\mathbf{2}$ & 1 & 0.054987 & 33 & 0.037835 & 1.453337 & 0.639037 \\
$\mathbf{1 \times 2}$ & 1 & 0.00848 & 33 & 0.037835 & 0.224118 & \\
\hline
\end{tabular}




\begin{tabular}{ccccccc}
\hline \multicolumn{7}{c}{ Baseline Motor Unit Activation - All Subjects } \\
\hline Effect & df & MS & d = Age & 2 = Gender & MS & F-level \\
& Effect & Effect & Error & Error & \\
\hline $\mathbf{1}$ & 1 & 88.3473 & 40 & 153.3827 & 0.575993 & 0.452337 \\
$\mathbf{2}$ & 1 & 273.8552 & 40 & 153.3827 & 1.785437 & 0.189035 \\
$\mathbf{1 \times 2}$ & 1 & 361.9273 & 40 & 153.3827 & 2.359636 & 0.132384 \\
\hline
\end{tabular}

\begin{tabular}{|c|c|c|c|c|c|c|}
\hline \multicolumn{7}{|c|}{ Peak Torque, Relative to Baseline, During Fatigue - All Subjects } \\
\hline & & & Age & $2=$ Gender & $3=$ Time & \\
\hline Effect & $\begin{array}{c}\text { df } \\
\text { Effect }\end{array}$ & $\begin{array}{c}\text { MS } \\
\text { Effect }\end{array}$ & $\begin{array}{c}\text { df } \\
\text { Error }\end{array}$ & $\begin{array}{l}\text { MS } \\
\text { Error }\end{array}$ & $\mathbf{F}$ & p-level \\
\hline 1 & 1 & 1.39342 & 38 & 0.598361 & 2.32873 & 0.135286 \\
\hline 2 & 1 & 0.086102 & 38 & 0.598361 & 0.1439 & 0.706549 \\
\hline 3 & 9 & 2.250287 & 342 & 0.032594 & 69.03976 & $0.000000^{*}$ \\
\hline $1 \times 2$ & 1 & 0.823553 & 38 & 0.598361 & 1.37635 & 0.248025 \\
\hline $1 \times 3$ & 9 & 0.140139 & 342 & 0.032594 & 4.29953 & $0.000026^{\star}$ \\
\hline $2 \times 3$ & 9 & 0.054172 & 342 & 0.032594 & 1.66201 & 0.096895 \\
\hline $1 \times 2 \times 3$ & 9 & 0.166805 & 342 & 0.032594 & 5.11765 & $0.000002^{*}$ \\
\hline
\end{tabular}

Peak Torque, Relative to Baseline, During Fatigue - Young Males vs. Young Females

\begin{tabular}{cccccccc}
\multicolumn{7}{c}{$\mathbf{1 = \text { Gender }}$ 2 = Time } \\
\hline Effect & $\begin{array}{c}\text { df } \\
\text { Effect }\end{array}$ & $\begin{array}{c}\text { MS } \\
\text { Effect }\end{array}$ & $\begin{array}{c}\text { df } \\
\text { Error }\end{array}$ & $\begin{array}{c}\text { MS } \\
\text { Error }\end{array}$ & F & p-level \\
& 1 & 0.721116 & 19 & 0.443517 & 1.62591 & 0.217642 \\
$\mathbf{1}$ & 9 & 1.688312 & 171 & 0.025285 & 66.77081 & $0.000000^{*}$ \\
$\mathbf{1}$ & 9 & 0.023449 & 171 & 0.025285 & 0.92936 & 0.501096 \\
\hline
\end{tabular}

Fatigue Index - Peak Torque - Young Males vs. Young Females

\begin{tabular}{|c|c|c|c|c|c|c|}
\hline Effect & $\begin{array}{c}\text { df } \\
\text { Effect }\end{array}$ & $\underset{\text { Effect }}{\text { MS }}$ & $\begin{array}{c}\text { df } \\
\text { Error }\end{array}$ & $\begin{array}{c}\text { MS } \\
\text { Error }\end{array}$ & $\bar{F}$ & p-level \\
\hline 1 & $\overline{1}$ & 55.80198 & $\overline{19}$ & 528.0676 & 0.105672 & 0.748679 \\
\hline
\end{tabular}

\begin{tabular}{ccccccc}
\hline \multicolumn{7}{l}{ Peak Torque, Relative to Baseline, During Fatigue - Elderly Males vs. Elderly Females } \\
\hline Effect & df & MS & df & MS & Fender & 2 = Time \\
& Effect & Effect & Error & Error & P-level \\
\hline $\mathbf{1}$ & 1 & 0.188539 & 19 & 0.753205 & 0.25032 & 0.622598 \\
$\mathbf{2}$ & 9 & 0.702114 & 171 & 0.039903 & 17.59554 & $0.000000^{*}$ \\
$\mathbf{1 \times 2}$ & 9 & 0.197478 & 171 & 0.039903 & 4.94895 & $0.000006^{*}$ \\
\hline
\end{tabular}




\begin{tabular}{ccccccc}
\hline \multicolumn{7}{c}{ Fatigue Index - Peak Torque - Elderly Males vs. Elderly Females } \\
$1=$ Gender
\end{tabular}

\begin{tabular}{|c|c|c|c|c|c|c|}
\hline \multicolumn{7}{|c|}{$\begin{array}{l}\text { Fatigue Index - Peak Torque - Young vs. Elderly } \\
\qquad 1=\text { Age }\end{array}$} \\
\hline Effect & $\begin{array}{c}\text { df } \\
\text { Effect }\end{array}$ & $\begin{array}{c}\text { MS } \\
\text { Effect }\end{array}$ & $\begin{array}{c}\text { df } \\
\text { Error }\end{array}$ & $\begin{array}{l}\text { MS } \\
\text { Error }\end{array}$ & $\mathbf{F}$ & p-level \\
\hline 1 & 1 & 3138.537 & 40 & 977.1213 & 3.212024 & 0.080665 \\
\hline
\end{tabular}

\begin{tabular}{cccccccc}
\hline \multicolumn{7}{c}{ Peak Torque, Relative to Baseline, During Recovery - All Subjects } \\
\hline Effect & $\begin{array}{c}\text { df } \\
\text { Effect }\end{array}$ & $\begin{array}{c}\text { MS } \\
\text { Effect }\end{array}$ & $\begin{array}{c}\text { df } \\
\text { Error }\end{array}$ & $\begin{array}{c}\text { MS } \\
\text { Error }\end{array}$ & Fender & 3 = Time & p-level \\
& $\mathbf{1}$ & 0.381244 & 38 & 0.313995 & 1.21417 & 0.277438 \\
$\mathbf{1}$ & 1 & 0.473681 & 38 & 0.313995 & 1.50856 & 0.226912 \\
$\mathbf{3}$ & 7 & 0.287047 & 266 & 0.019901 & 14.42407 & $0.000000^{*}$ \\
$\mathbf{1 \times 2}$ & 1 & 0.014406 & 38 & 0.313995 & 0.04588 & 0.831541 \\
$\mathbf{1 \times 3}$ & 7 & 0.028706 & 266 & 0.019901 & 1.44246 & 0.188411 \\
$\mathbf{2} \times 3$ & 7 & 0.008014 & 266 & 0.019901 & 0.40268 & 0.900255 \\
$\mathbf{1 \times 2 \times 3}$ & 7 & 0.016049 & 266 & 0.019901 & 0.80649 & 0.582524 \\
\hline
\end{tabular}

\begin{tabular}{|c|c|c|c|c|c|c|}
\hline \multicolumn{7}{|c|}{$\begin{array}{l}\text { Maximum Rate of Torque Development, Relative to Baseline, During Fatigue - All Subjects } \\
\qquad 1=A a e \quad 2=\text { Gender } 3=\text { Time }\end{array}$} \\
\hline Effect & $\begin{array}{c}\text { df } \\
\text { Effect }\end{array}$ & $\begin{array}{c}\text { MS } \\
\text { Effect }\end{array}$ & $\begin{array}{l}\text { df } \\
\text { Error }\end{array}$ & $\begin{array}{l}\text { MS } \\
\text { Error }\end{array}$ & $\bar{F}$ & p-level \\
\hline 1 & 1 & 0.463243 & 38 & 0.688827 & 0.67251 & 0.417293 \\
\hline 2 & 1 & 0.289978 & 38 & 0.688827 & 0.42097 & 0.520354 \\
\hline 3 & 9 & 1.999255 & 342 & 0.037681 & 53.05668 & $0.000000^{\star}$ \\
\hline $1 \times 2$ & 1 & 0.723506 & 38 & 0.688827 & 1.05034 & 0.031191 \\
\hline $1 \times 3$ & 9 & 0.147803 & 342 & 0.037681 & 3.92243 & $0.000092^{\star}$ \\
\hline $2 \times 3$ & 9 & 0.044143 & 342 & 0.037681 & 1.17147 & 0.312316 \\
\hline $1 \times 2 \times 3$ & 9 & 0.105961 & 342 & 0.037681 & 2.81203 & $0.003367^{\star}$ \\
\hline
\end{tabular}




\begin{tabular}{|c|c|c|c|c|c|c|}
\hline \multicolumn{7}{|c|}{$\begin{array}{l}\text { Half Relaxation Time, Relative to Baseline, During Fatigue - Young Males vs. Elderly Males } \\
\qquad \begin{array}{l}1=\mathrm{Age} \quad 2=\text { Time }\end{array}\end{array}$} \\
\hline Effect & $\begin{array}{c}\text { df } \\
\text { Effect }\end{array}$ & $\begin{array}{c}\text { MS } \\
\text { Effect }\end{array}$ & $\begin{array}{c}\text { df } \\
\text { Error }\end{array}$ & $\begin{array}{l}\text { MS } \\
\text { Error }\end{array}$ & $\bar{F}$ & p-level \\
\hline 1 & 1 & 0.186414 & 19 & 0.725768 & 0.256851 & 0.618123 \\
\hline 2 & 9 & 0.267526 & 171 & 0.100703 & 2.656581 & $0.006591^{*}$ \\
\hline $1 \times 2$ & 9 & 0.086371 & 171 & 0.100703 & 0.857684 & 0.564316 \\
\hline
\end{tabular}

Talf Relaxation Time, Relative to Baseline, During Fatigue - Young Females vs. Elderly Female

\begin{tabular}{|c|c|c|c|c|c|c|}
\hline Effect & $\begin{array}{c}\text { df } \\
\text { Effect }\end{array}$ & $\begin{array}{c}\text { MS } \\
\text { Effect }\end{array}$ & $\begin{array}{c}\text { df } \\
\text { Error }\end{array}$ & $\begin{array}{c}\text { MS } \\
\text { Error }\end{array}$ & $\bar{F}$ & p-level \\
\hline 1 & 1 & 0.444087 & 19 & 0.479468 & 0.926208 & 0.347939 \\
\hline 2 & 9 & 0.12205 & 171 & 0.065425 & 1.8655 & 0.06013 \\
\hline $1 \times 2$ & 9 & 0.040932 & 171 & 0.065425 & 0.625631 & 0.774135 \\
\hline
\end{tabular}

\begin{tabular}{|c|c|c|c|c|c|c|}
\hline \multicolumn{7}{|c|}{$\begin{array}{l}\text { Half Relaxation Time, Relative to Baseline, During Fatigue - Males vs. Females } \\
\qquad 1=\text { Gender } \quad 2=\text { Time } \\
\end{array}$} \\
\hline Effect & $\begin{array}{c}\text { df } \\
\text { Effect }\end{array}$ & $\begin{array}{c}\text { MS } \\
\text { Effect }\end{array}$ & $\begin{array}{c}\text { df } \\
\text { Error }\end{array}$ & $\begin{array}{l}\text { MS } \\
\text { Error }\end{array}$ & $\bar{F}$ & p-level \\
\hline 1 & 1 & 3.94269 & 40 & 0.58825 & 6.702411 & $0.013361^{*}$ \\
\hline 2 & 9 & 0.148068 & 360 & 0.082093 & 1.803659 & 0.066237 \\
\hline $1 \times 2$ & 9 & 0.243497 & 360 & 0.082093 & 2.9661 & $0.002043^{\star}$ \\
\hline
\end{tabular}

\begin{tabular}{cccccccc}
\hline \multicolumn{7}{c}{ M-wave Area, Relative to Baseline, During Fatigue - All Subjects } \\
\hline Effect & $\begin{array}{c}\text { df } \\
\text { Effect }\end{array}$ & $\begin{array}{c}\text { MS } \\
\text { Effect }\end{array}$ & $\begin{array}{c}\text { df } \\
\text { Error }\end{array}$ & $\begin{array}{c}\text { MS } \\
\text { Error }\end{array}$ & Fender & 3= Time & p-level \\
& 1 & 0.471942 & 29 & 0.592244 & 0.796871 & 0.379378 \\
\hline $\mathbf{1}$ & 1 & 0.545727 & 29 & 0.592244 & 0.921455 & 0.345029 \\
$\mathbf{2}$ & 9 & 0.092053 & 261 & 0.018016 & 5.109651 & $0.000002^{*}$ \\
$\mathbf{3}$ & 1 & 0.033333 & 29 & 0.592244 & 0.056282 & 0.81414 \\
$\mathbf{1 \times 2}$ & 9 & 0.057874 & 261 & 0.018016 & 3.212447 & $0.001037^{*}$ \\
$\mathbf{1 \times 3}$ & 9 & 0.036502 & 261 & 0.018016 & 2.026154 & $0.036824^{*}$ \\
$\mathbf{2} \times \mathbf{3}$ & 9 & 0.005489 & 261 & 0.018016 & 0.304675 & 0.972915 \\
$\mathbf{1 \times 2 \times 3}$ & 9 &
\end{tabular}




\begin{tabular}{|c|c|c|c|c|c|c|}
\hline \multicolumn{7}{|c|}{$\begin{array}{l}\text { M-wave Area, Relative to Baseline, During Fatigue - Young Males vs. Young Females } \\
\qquad 1=\text { Gender } \quad 2=\text { Time }\end{array}$} \\
\hline Effect & $\begin{array}{c}\text { df } \\
\text { Effect }\end{array}$ & $\begin{array}{c}\text { MS } \\
\text { Effect }\end{array}$ & $\begin{array}{c}\text { df } \\
\text { Error }\end{array}$ & $\begin{array}{c}\text { MS } \\
\text { Error }\end{array}$ & $F$ & p-level \\
\hline 1 & 1 & 0.159206 & 15 & 0.365976 & 0.435018 & 0.519536 \\
\hline 2 & 9 & 0.009409 & 135 & 0.012124 & 0.776069 & 0.638725 \\
\hline $1 \times 2$ & 9 & 0.027074 & 135 & 0.12124 & 2.233099 & $0.023446^{*}$ \\
\hline
\end{tabular}

\begin{tabular}{|c|c|c|c|c|c|c|}
\hline \multicolumn{7}{|c|}{$\begin{array}{l}\text { M-wave Area, Relative to Baseline, During Fatigue - Young Males } \\
\qquad 1 \text { = Time }\end{array}$} \\
\hline Effect & $\begin{array}{c}\text { df } \\
\text { Effect }\end{array}$ & $\begin{array}{c}\text { MS } \\
\text { Effect }\end{array}$ & $\begin{array}{l}\text { df } \\
\text { Error }\end{array}$ & $\begin{array}{l}\text { MS } \\
\text { Error }\end{array}$ & $\bar{F}$ & p-level \\
\hline 1 & 9 & 0.032214 & 72 & 0.011234 & 2.867517 & $0.006002^{*}$ \\
\hline
\end{tabular}

\begin{tabular}{ccccccc}
\hline \multicolumn{7}{c}{ M-wave Area, Relative to Baseline, During Fatigue - Young Females } \\
$1=$ Time
\end{tabular}

\begin{tabular}{cccccccc}
\hline \multicolumn{7}{c}{ M-wave Area, Relative to Baseline, During Fatigue - Elderly Males vs. Elderly Females } \\
\multicolumn{7}{c}{} & \multicolumn{7}{c}{ 1= Gender } & 2 = Time \\
\hline Effect & df & MS & df & MS & F & p-level \\
& Effect & Effect & Error & Error & \\
\hline 1 & 1 & 0.412613 & 14 & 0.834675 & 0.49434 & 0.493525 \\
$\mathbf{2}$ & 9 & 0.136877 & 126 & 0.024328 & 5.626313 & $0.000002^{\star}$ \\
$1 \times 2$ & 9 & 0.015255 & 126 & 0.024328 & 0.627044 & 0.772208 \\
\hline
\end{tabular}

\begin{tabular}{|c|c|c|c|c|c|c|}
\hline \multicolumn{7}{|c|}{$\begin{array}{l}\text { M-wave Amplitude, Relative to Baseline, During Fatigue - All Subjects } \\
\qquad 1=\text { Age } \quad 2=\text { Gender } \quad 3=\text { Time }\end{array}$} \\
\hline Effect & $\begin{array}{c}\text { df } \\
\text { Effect }\end{array}$ & $\begin{array}{c}\text { MS } \\
\text { Effect }\end{array}$ & $\begin{array}{c}\text { df } \\
\text { Error }\end{array}$ & $\begin{array}{l}\text { MS } \\
\text { Error }\end{array}$ & $\bar{F}$ & p-level \\
\hline 1 & 1 & 1.040832 & 29 & 0.526021 & 1.97869 & 0.170155 \\
\hline 2 & 1 & 2.00787 & 29 & 0.526021 & 3.81709 & 0.060439 \\
\hline 3 & 9 & 0.329086 & 261 & 0.017232 & 19.09769 & $0.000000^{\star}$ \\
\hline $1 \times 2$ & 1 & 0.048687 & 29 & 0.526021 & 0.09256 & 0.763124 \\
\hline $1 \times 3$ & 9 & 0.067309 & 261 & 0.017232 & 3.90611 & $0.000113^{\star}$ \\
\hline $2 \times 3$ & 9 & 0.049667 & 261 & 0.017232 & 2.88228 & 0.002907 \\
\hline $1 \times 2 \times 3$ & 9 & 0.013058 & 261 & 0.017232 & 0.75779 & 0.655617 \\
\hline
\end{tabular}




\begin{tabular}{cccccccc}
\hline \multicolumn{7}{c}{ M-wave Amplitude, Relative to Baseline, During Fatigue - Young Males vs. Young Females } \\
\hline \multicolumn{7}{c}{ 1 = Gender } & 2=Time \\
\hline Effect & df & MS & df & MS & F & p-level \\
& Effect & Effect & Error & Error & 0.16159 \\
\hline $\mathbf{1}$ & 1 & 0.736665 & 15 & 0.339807 & 2.167892 & $0.000000^{*}$ \\
$\mathbf{2}$ & 9 & 0.072212 & 135 & 0.011531 & 6.262409 & $0.000215^{\star}$ \\
$\mathbf{1 \times 2}$ & 9 & 0.044691 & 135 & 0.011531 & 3.875689 &
\end{tabular}

\begin{tabular}{cccccccc}
\hline \multicolumn{7}{l}{ M-wave Amplitude, Relative to Baseline, During Fatigue - Elderly Males vs. Elderly Females } \\
\hline \multicolumn{7}{c}{ 1 = Gender } & 2 = Time \\
\hline Effect & df & MS & df & MS & F & p-level \\
& Effect & Effect & Error & Error & \\
\hline 1 & 1 & 1.303691 & 14 & 0.725536 & 1.79686 & 0.20144 \\
$\mathbf{2}$ & 9 & 0.317184 & 126 & 0.02334 & 13.58993 & $0.000000^{*}$ \\
$\mathbf{1 \times 2}$ & 9 & 0.018775 & 126 & 0.02334 & 0.80441 & 0.612921 \\
\hline
\end{tabular}

\begin{tabular}{|c|c|c|c|c|c|c|}
\hline \multicolumn{7}{|c|}{$\begin{array}{l}\text { M-wave Duration, Relative to Baseline, During Fatigue - All Subjects } \\
\qquad 1=A a e \quad 2=\text { Gender } 3=\text { Time }\end{array}$} \\
\hline Effect & $\begin{array}{c}\text { df } \\
\text { Effect }\end{array}$ & $\begin{array}{c}\text { MS } \\
\text { Effect }\end{array}$ & $\begin{array}{l}\text { df } \\
\text { Error }\end{array}$ & $\begin{array}{c}\text { MS } \\
\text { Error }\end{array}$ & $\bar{F}$ & p-level \\
\hline 1 & 1 & 0.480878 & 29 & 0.133235 & 3.609249 & 0.067445 \\
\hline 2 & 1 & 0.973197 & 29 & 0.133235 & 7.304378 & $0.011377^{*}$ \\
\hline 3 & 9 & 0.107196 & 261 & 0.012351 & 8.678852 & $0.000000^{*}$ \\
\hline $1 \times 2$ & 1 & 0.045871 & 29 & 0.133235 & 0.344285 & 0.561907 \\
\hline $1 \times 3$ & 9 & 0.003607 & 261 & 0.012351 & 0.29034 & 0.976571 \\
\hline $2 \times 3$ & 9 & 0.010921 & 261 & 0.012351 & 0.884168 & 0.53989 \\
\hline $1 \times 2 \times 3$ & 9 & 0.009002 & 261 & 0.012351 & 0.728787 & 0.682368 \\
\hline
\end{tabular}

\begin{tabular}{|c|c|c|c|c|c|c|}
\hline \multicolumn{7}{|c|}{$\begin{array}{l}\text { MVC, Relative to Baseline, During Fatigue - Young Males vs. Young Females } \\
\qquad 1=\text { Gender } \quad 2=\text { Time }\end{array}$} \\
\hline Effect & $\begin{array}{c}\text { df } \\
\text { Effect }\end{array}$ & $\begin{array}{c}\text { MS } \\
\text { Effect }\end{array}$ & $\begin{array}{c}\text { df } \\
\text { Error }\end{array}$ & $\begin{array}{l}\text { MS } \\
\text { Error }\end{array}$ & $\bar{F}$ & p-level \\
\hline 1 & 1 & 0.065136 & 19 & 0.06364 & 1.02351 & 0.3244 \\
\hline 2 & 9 & 0.279072 & 171 & 0.006649 & 41.97403 & $0.000000^{*}$ \\
\hline $1 \times 2$ & 9 & 0.012353 & 171 & 0.006649 & 1.85794 & 0.06134 \\
\hline
\end{tabular}

\begin{tabular}{ccccccc}
\hline 1= Gendel & \multicolumn{7}{c}{ Fatigue Index - MVC - Young Males vs. Young Females } \\
\hline Effect & $\begin{array}{c}\text { df } \\
\text { Effect }\end{array}$ & $\begin{array}{c}\text { MS } \\
\text { Effect }\end{array}$ & $\begin{array}{c}\text { df } \\
\text { Error }\end{array}$ & $\begin{array}{c}\text { MS } \\
\text { Error }\end{array}$ & F & p-level \\
\hline 1 & 1 & 388.2708 & 19 & 145.8374 & 2.662354 & 0.119213 \\
\hline
\end{tabular}




\begin{tabular}{|c|c|c|c|c|c|c|}
\hline \multicolumn{7}{|c|}{ MVC, Relative to Baseline, During Fatigue - Elderly Males vs. Elderly Females } \\
\hline Effect & $\begin{array}{c}\text { df } \\
\text { Effect }\end{array}$ & $\begin{array}{c}\text { MS } \\
\text { Effect }\end{array}$ & $\begin{array}{c}\text { df } \\
\text { Error }\end{array}$ & $\begin{array}{c}\text { MS } \\
\text { Error }\end{array}$ & $\bar{F}$ & p-level \\
\hline 1 & 1 & 0.006838 & 19 & 0.198681 & 0.034418 & 0.854786 \\
\hline 2 & 9 & 0.049703 & 171 & 0.00829 & 5.99578 & $0.000000^{*}$ \\
\hline $1 \times 2$ & 9 & 0.001243 & 171 & 0.00829 & 0.149982 & 0.99799 \\
\hline
\end{tabular}

\begin{tabular}{ccccccc}
\hline \multicolumn{7}{c}{ Fatigue Index - MVC - Elderly Males vs. Elderly Females } \\
$1=$ Gender
\end{tabular}

\begin{tabular}{cccccccc}
\hline \multicolumn{7}{c}{ MVC, Relative to Baseline, During Fatigue - All Subjects } \\
\hline Effect & $\begin{array}{c}\text { df } \\
\text { Effect }\end{array}$ & $\begin{array}{c}\text { MS } \\
\text { Effect }\end{array}$ & $\begin{array}{c}\text { df } \\
\text { Error }\end{array}$ & $\begin{array}{c}\text { MS } \\
\text { Error }\end{array}$ & pender & 3= Time \\
& 1 & 1.138453 & 38 & 0.131161 & 8.67985 & \\
\hline $\mathbf{1}$ & 1 & 0.014882 & 38 & 0.131161 & 0.11346 & $0.005470^{*}$ \\
$\mathbf{2}$ & 9 & 0.27781 & 342 & 0.007469 & 37.19398 & 0.738087 \\
$\mathbf{3}$ & 1 & 0.057092 & 38 & 0.131161 & 0.43528 & $0.000000^{*}$ \\
$\mathbf{1 \times 2}$ & 9 & 0.050966 & 342 & 0.007469 & 6.82346 & $0.000000^{*}$ \\
$\mathbf{1 \times 3}$ & 9 & 0.006865 & 342 & 0.007469 & 0.91913 & 0.508468 \\
$\mathbf{2 \times 3}$ & 9 & 0.006731 & 342 & 0.007469 & 0.90116 & 0.524357 \\
$\mathbf{1 \times 2 \times 3}$ & 9 & 0 &
\end{tabular}

\begin{tabular}{|c|c|c|c|c|c|c|}
\hline \multicolumn{7}{|c|}{$\begin{array}{l}\text { Fatigue Index - MVC - Young Males vs. Elderly Males } \\
\qquad 1=\mathrm{Age}\end{array}$} \\
\hline Effect & $\begin{array}{c}\text { df } \\
\text { Effect }\end{array}$ & $\begin{array}{c}\text { MS } \\
\text { Effect }\end{array}$ & $\begin{array}{c}\text { df } \\
\text { Error }\end{array}$ & $\begin{array}{c}\text { MS } \\
\text { Error }\end{array}$ & $F$ & p-level \\
\hline 1 & 1 & 2095.151 & 18 & 112.5761 & 18.61098 & 0.00418 \\
\hline
\end{tabular}

\begin{tabular}{ccccccc}
\hline \multicolumn{7}{c}{ Fatigue Index - MVC - Young Females vs. Elderly Females } \\
1 = Age
\end{tabular}




\begin{tabular}{cccccccc}
\hline \multicolumn{7}{c}{ MVC, Relative to Baseline, During Recovery - All Subjects } \\
\hline Effect & $\begin{array}{c}\text { df } \\
\text { Effect }\end{array}$ & $\begin{array}{c}\text { MS } \\
\text { Effect }\end{array}$ & $\begin{array}{c}\text { df } \\
\text { Error }\end{array}$ & $\begin{array}{c}\text { MS } \\
\text { Error }\end{array}$ & Fender & 3= Time & p-level \\
& 1 & 0.00101 & 38 & 0.022087 & 0.045717 & 0.831835 \\
\hline $\mathbf{1}$ & 1 & 0.012727 & 38 & 0.022087 & 0.57624 & 0.452473 \\
$\mathbf{2}$ & 1 & 0.014674 & 38 & 0.012471 & 1.176675 & 0.284868 \\
$\mathbf{3}$ & 1 & 0.001352 & 38 & 0.022087 & 0.061196 & 0.805948 \\
$\mathbf{1 \times 2}$ & 1 & 0.030362 & 38 & 0.012471 & 2.434632 & 0.126972 \\
$\mathbf{1 \times 3}$ & 1 & 0.000056 & 38 & 0.012471 & 0.004496 & 0.946895 \\
$\mathbf{2 \times 3}$ & 1 & 0.00242 & 38 & 0.012471 & 0.19405 & 0.662063 \\
$\mathbf{1 \times 2 \times 3}$ & 1 & & & & & \\
\hline
\end{tabular}

\begin{tabular}{|c|c|c|c|c|c|c|}
\hline \multicolumn{7}{|c|}{$\begin{array}{l}\text { AEMG, Relative to Baseline, During Fatigue - All Subjects } \\
\qquad 1=\text { Age } \quad 2=\text { Gender } \quad 3=\text { Time }\end{array}$} \\
\hline Effect & $\begin{array}{c}\text { df } \\
\text { Effect }\end{array}$ & $\begin{array}{c}\text { MS } \\
\text { Effect }\end{array}$ & $\begin{array}{c}\text { df } \\
\text { Error }\end{array}$ & $\begin{array}{c}\text { MS } \\
\text { Error }\end{array}$ & $F$ & p-level \\
\hline 1 & 1 & 0.135561 & 29 & 0.274186 & 0.494412 & 0.487573 \\
\hline 2 & 1 & 0.403343 & 29 & 0.274186 & 1.471059 & 0.234966 \\
\hline 3 & 9 & 0.056817 & 261 & 0.020677 & 2.747896 & $0.004394^{*}$ \\
\hline $1 \times 2$ & 1 & 0.036482 & 29 & 0.274186 & 0.133057 & 0.71793 \\
\hline $1 \times 3$ & 9 & 0.041821 & 261 & 0.020677 & 2.022637 & $0.037188^{*}$ \\
\hline $2 \times 3$ & 9 & 0.034142 & 261 & 0.020677 & 1.651251 & 0.101136 \\
\hline $1 \times 2 \times 3$ & 9 & 0.016407 & 261 & 0.020677 & 0.79349 & 0.622609 \\
\hline
\end{tabular}

\begin{tabular}{ccccccc}
\hline \multicolumn{7}{c}{ MUA During Fatigue - Young Males vs. Young Females } \\
\hline Effect & df & MS & df & MS & 2 = Time \\
& Effect & Effect & Error & Error & F & p-level \\
\hline 1 & 1 & 4637.863 & 16 & 1110.134 & 4.17775 & 0.05778 \\
2 & 9 & 1487.142 & 144 & 130.122 & 11.42879 & $0.000000^{*}$ \\
$1 \times 2$ & 9 & 84.811 & 144 & 130.122 & 0.65177 & 0.751068 \\
\hline
\end{tabular}

\begin{tabular}{cccccccc}
\hline \multicolumn{7}{c}{ MUA During Fatigue - Elderly Males vs. Elderly Females } \\
\hline Effect & df & MS & df & MS & 2 = Time \\
& Effect & Effect & Error & Error & F & p-level \\
\hline $\mathbf{1}$ & 1 & 2601.967 & 18 & 1698.315 & 1.532088 & 0.231702 \\
$\mathbf{2}$ & 9 & 264.565 & 162 & 127.53 & 2.074536 & $0.034630^{*}$ \\
$\mathbf{1 x 2}$ & 9 & 79.323 & 162 & 127.53 & 0.621994 & 0.77714 \\
\hline
\end{tabular}




\begin{tabular}{|c|c|c|c|c|c|c|}
\hline \multicolumn{7}{|c|}{ MUA During Fatigue - All Subjects } \\
\hline & & & Age & 2 = Gender & $3=$ Time & \\
\hline Effect & $\begin{array}{c}\mathrm{df} \\
\text { Effect }\end{array}$ & $\begin{array}{c}\text { MS } \\
\text { Effect }\end{array}$ & $\begin{array}{c}\text { df } \\
\text { Error }\end{array}$ & $\begin{array}{c}\text { MS } \\
\text { Error }\end{array}$ & $\mathbf{F}$ & p-level \\
\hline 1 & 1 & 347.551 & 34 & 1421.524 & 0.24449 & 0.62416 \\
\hline 2 & 1 & 7143.502 & 34 & 1421.524 & 5.02524 & $0.031616^{*}$ \\
\hline 3 & 9 & 1321.434 & 306 & 128.75 & 10.26357 & $0.000000^{*}$ \\
\hline $1 \times 2$ & 1 & 205.888 & 34 & 1421.524 & 0.14484 & 0.705887 \\
\hline $1 \times 3$ & 9 & 496.065 & 306 & 128.75 & 3.85294 & $0.000122^{\star}$ \\
\hline $2 \times 3$ & 9 & 122.166 & 306 & 128.75 & 0.94887 & 0.482839 \\
\hline $1 \times 2 \times 3$ & 9 & 42.262 & 306 & 128.75 & 0.32825 & 0.965333 \\
\hline
\end{tabular}

\begin{tabular}{cccccccc}
\hline \multicolumn{7}{c}{ AEMG:M-wave Area Ratio During Fatigue - All Subjects } \\
\hline Effect & df & MS & 1 = Age & 2 = Gender & 3=Time & MS & F-level \\
& Effect & Effect & Error & Error & \\
\hline $\mathbf{1}$ & 1 & 0.011665 & 29 & 1.490335 & 0.007827 & 0.930111 \\
$\mathbf{2}$ & 1 & 0.471132 & 29 & 1.490335 & 0.316125 & 0.578266 \\
$\mathbf{3}$ & 9 & 0.082733 & 261 & 0.084886 & 0.974645 & 0.461366 \\
$\mathbf{1 \times 2}$ & 1 & 0.001207 & 29 & 1.490335 & 0.00081 & 0.977494 \\
$\mathbf{1 \times 3}$ & 9 & 0.332533 & 261 & 0.084886 & 3.917427 & $0.000109^{*}$ \\
$\mathbf{2} \times \mathbf{3}$ & 9 & 0.106532 & 261 & 0.084886 & 1.255009 & 0.261935 \\
$\mathbf{1 \times 2 \times 3}$ & 9 & 0.028629 & 261 & 0.084886 & 0.337271 & 0.961942 \\
\hline
\end{tabular}

\begin{tabular}{|c|c|c|c|c|c|c|}
\hline \multicolumn{7}{|c|}{ Regression Summary for Dependent Variable FI-MVC, Young Males } \\
\hline $\mathbf{N}=10$ & Beta & $\begin{array}{l}\text { St. Err. } \\
\text { of Beta }\end{array}$ & $\mathbf{B}$ & $\begin{array}{c}\text { St. Err. } \\
\text { of B }\end{array}$ & $t(7)$ & p-level \\
\hline Intercept & & & 7.938322 & 30.90467 & 0.256865 & 0.804669 \\
\hline AP & 0.043385 & 0.339899 & 7.963742 & 6.23922 & 1.276401 & 0.242527 \\
\hline C-MVC & 0.062666 & 0.339899 & 0.01051 & 0.05701 & 0.184368 & 0.858952 \\
\hline
\end{tabular}

\begin{tabular}{|c|c|c|c|c|c|c|}
\hline \multicolumn{7}{|c|}{$\begin{array}{l}\text { Regression Summary for Dependent Variable FI-MVC, Young Females } \\
\qquad \begin{array}{l}R=0.5682 \quad R^{\wedge} 2=0.3228 \quad F(2,8)=1.9074 \quad p<0.21021\end{array}\end{array}$} \\
\hline$N=11$ & Beta & $\begin{array}{l}\text { St. Err. } \\
\text { of Beta }\end{array}$ & $\bar{B}$ & $\begin{array}{l}\text { St. Err. } \\
\text { of B }\end{array}$ & $t(7)$ & p-level \\
\hline Intercept & & & 114.0059 & 62.27641 & 1.83064 & 0.104534 \\
\hline $\mathbf{A P}$ & -0.5601 & 0.313882 & -21.2738 & 11.92192 & -1.78443 & 0.112189 \\
\hline C-MVC & 0.02077 & 0.313882 & 0.0101 & 0.15195 & 0.06619 & 0.948854 \\
\hline
\end{tabular}




\begin{tabular}{|c|c|c|c|c|c|c|}
\hline \multicolumn{7}{|c|}{ Regression Summary for Dependent Variable FI-MVC, Elderly Males } \\
\hline & & $=0.6985$ & $\mathbf{R}^{\wedge} \mathbf{2}=0.4$ & $79 F(2,7$ & $p<0.09605$ & \\
\hline$N=10$ & Beta & $\begin{array}{l}\text { St. Err. } \\
\text { of Beta }\end{array}$ & $\mathbf{B}$ & $\begin{array}{c}\text { St. Err. } \\
\text { of } B\end{array}$ & $\bar{t}(7)$ & p-level \\
\hline Intercept & & & 60.1964 & 29.66439 & 2.02925 & 0.082004 \\
\hline AP & -0.567 & 0.272944 & -14.1651 & 6.81855 & -2.07744 & 0.076374 \\
\hline C-MVC & 0.3386 & 0.272944 & 0.073 & 0.05883 & 1.24081 & 0.254646 \\
\hline
\end{tabular}

\begin{tabular}{|c|c|c|c|c|c|c|}
\hline \multicolumn{7}{|c|}{ Regression Summary for Dependent Variable FI-MVC, Elderly Females } \\
\hline $\mathbf{N}=\mathbf{9}$ & Beta & $\begin{array}{l}\text { St. Err. } \\
\text { of Beta }\end{array}$ & B & $\begin{array}{c}\text { St. Err. } \\
\text { of } B\end{array}$ & $\mathbf{t}(7)$ & p-level \\
\hline Intercept & & & -1.39766 & 89.93768 & -0.01554 & 0.988105 \\
\hline AP & 0.05908 & 0.449286 & 2.71585 & 20.65294 & 0.131499 & 0.899679 \\
\hline C-MVC & 0.267154 & 0.449286 & 0.07839 & 0.13183 & 0.594618 & 0.573822 \\
\hline
\end{tabular}

\begin{tabular}{|c|c|c|c|c|c|c|}
\hline \multicolumn{7}{|c|}{$\begin{array}{l}\text { Regression Summary for Dependent Variable FI-MVC, Young Males and Young Females (1) } \\
\qquad R=0.3505 \quad R^{\wedge} 2=0.1229 \quad F(1,19)=2.6624 \quad p<0.1192\end{array}$} \\
\hline$N=21$ & Beta & $\begin{array}{l}\text { St. Err. } \\
\text { of Beta }\end{array}$ & B & $\begin{array}{l}\text { St. Err. } \\
\text { of B }\end{array}$ & $t(7)$ & p-level \\
\hline $\begin{array}{l}\text { Intercept } \\
\text { Gender }\end{array}$ & 0.3505 & 0.214856 & $\begin{array}{c}36.08447 \\
8.60955\end{array}$ & $\begin{array}{l}3.641146 \\
5.276522\end{array}$ & $\begin{array}{l}9.910195 \\
1.631672\end{array}$ & $\begin{array}{c}0 \\
0.119213 \\
\end{array}$ \\
\hline
\end{tabular}

\begin{tabular}{|c|c|c|c|c|c|c|}
\hline \multicolumn{7}{|c|}{$\begin{array}{l}\text { Regression Summary for Dependent Variable Fl-MVC, Young Males and Young Females (2) } \\
\qquad R=0.3712 \quad R^{\wedge} 2=0.1378 \quad F(2,18)=1.4391 \quad p<0.26316\end{array}$} \\
\hline$N=21$ & Beta & $\begin{array}{l}\text { St. Err. } \\
\text { of Beta }\end{array}$ & B & $\begin{array}{l}\text { St. Err. } \\
\text { of } B\end{array}$ & $t(7)$ & p-level \\
\hline Intercept & & & 29.65951 & 12.0821 & 2.454831 & 0.024498 \\
\hline Gender & 0.17777 & 0.378863 & 4.36589 & 9.30429 & 0.469234 & 0.644536 \\
\hline C-MVC & 0.2116 & 0.378863 & 0.0331 & 0.05924 & 0.558752 & 0.583216 \\
\hline
\end{tabular}

\begin{tabular}{|c|c|c|c|c|c|c|}
\hline \multicolumn{7}{|c|}{$\begin{array}{l}\text { Regression Summary for Dependent Variable FI-MVC, Young Males and Young Females (3) } \\
\qquad R=0.3717 \quad R^{\wedge} 2=0.1382 F(3,17)=0.90894 \quad p<0.45737\end{array}$} \\
\hline$N=21$ & Beta & $\begin{array}{l}\text { St. Err. } \\
\text { of Beta }\end{array}$ & $\bar{B}$ & $\begin{array}{l}\text { St. Err. } \\
\text { of B }\end{array}$ & $t(7)$ & p-level \\
\hline Intercept & & & 31.67348 & 26.56512 & 1.192296 & 0.2495 \\
\hline Gender & 0.1878 & 0.40695 & 4.61241 & 9.99406 & 0.461515 & 0.6502 \\
\hline AP & -0.02148 & 0.250451 & -0.53008 & 6.17939 & -0.08578 & 0.9326 \\
\hline C-MVC & 0.210908 & 0.38987 & 0.03298 & 0.06096 & 0.54097 & 0.5955 \\
\hline
\end{tabular}


Regression Summary for Dependent Variable FI-MVC, Young Males and Elderly Males (1) $R=0.7222 \quad R^{\wedge} 2=0.5215 \quad F(1,18)=19.625 \quad p<0.00032$

\begin{tabular}{ccccccc}
\hline $\mathbf{N}=20$ & Beta & $\begin{array}{c}\text { St. Err. } \\
\text { of Beta }\end{array}$ & B & $\begin{array}{c}\text { St. Err. } \\
\text { of B }\end{array}$ & $\mathbf{t}(7)$ & p-level \\
\hline $\begin{array}{c}\text { Intercept } \\
\text { Age }\end{array}$ & -0.7222 & 0.163029 & -0.4377 & 0.0988 & 10.4232 & 0 \\
\hline
\end{tabular}

\begin{tabular}{|c|c|c|c|c|c|c|}
\hline \multicolumn{7}{|c|}{$\begin{array}{l}\text { Regression Summary for Dependent Variable FI-MVC, Young Males and Elderly Males (2) } \\
\qquad R=0.7332 \quad R^{\wedge} 2=0.53764 \quad F(2,17)=9.8843 \quad p<0.00142\end{array}$} \\
\hline$N=\mathbf{2 0}$ & Beta & $\begin{array}{l}\text { St. Err. } \\
\text { of Beta }\end{array}$ & B & $\begin{array}{l}\text { St. Err. } \\
\text { of } B\end{array}$ & $t(7)$ & p-level \\
\hline Intercept & & & 42.06719 & 18.64517 & 2.2562 & 0.037519 \\
\hline Age & -0.5847 & 0.24333 & -0.3544 & 0.14749 & -2.403 & $0.027956^{*}$ \\
\hline C-MVC & 0.1869 & 0.24333 & 0.03576 & 0.04654 & 0.76843 & 0.452774 \\
\hline
\end{tabular}

\begin{tabular}{|c|c|c|c|c|c|c|}
\hline \multicolumn{7}{|c|}{ Regression Summary for Dependent Variable FI-MVC, Young Males and Elderly Males (3) } \\
\hline \multicolumn{3}{|c|}{$R=0.73326$} & \multicolumn{2}{|c|}{$R^{\wedge} 2=0.53767$} & $p<0.00534$ & \multirow[b]{2}{*}{ p-level } \\
\hline $\mathbf{N}=\mathbf{2 1}$ & Beta & $\begin{array}{l}\text { St. Err. } \\
\text { of Beta }\end{array}$ & $\mathbf{B}$ & $\begin{array}{c}\text { St. Err. } \\
\text { of B }\end{array}$ & $t(7)$ & \\
\hline Intercept & & & 41.37065 & 29.39314 & 1.40749 & 0.178412 \\
\hline Age & -0.5819 & 0.2657 & -0.3527 & 0.16107 & -2.19012 & $0.043675^{\star}$ \\
\hline $\mathbf{A P}$ & 0.00599 & 0.191371 & 0.1568 & 5.00839 & 0.03132 & 0.975402 \\
\hline C-MVC & 0.18697 & 0.250811 & 0.03576 & 0.04797 & 0.74549 & 0.466788 \\
\hline
\end{tabular}

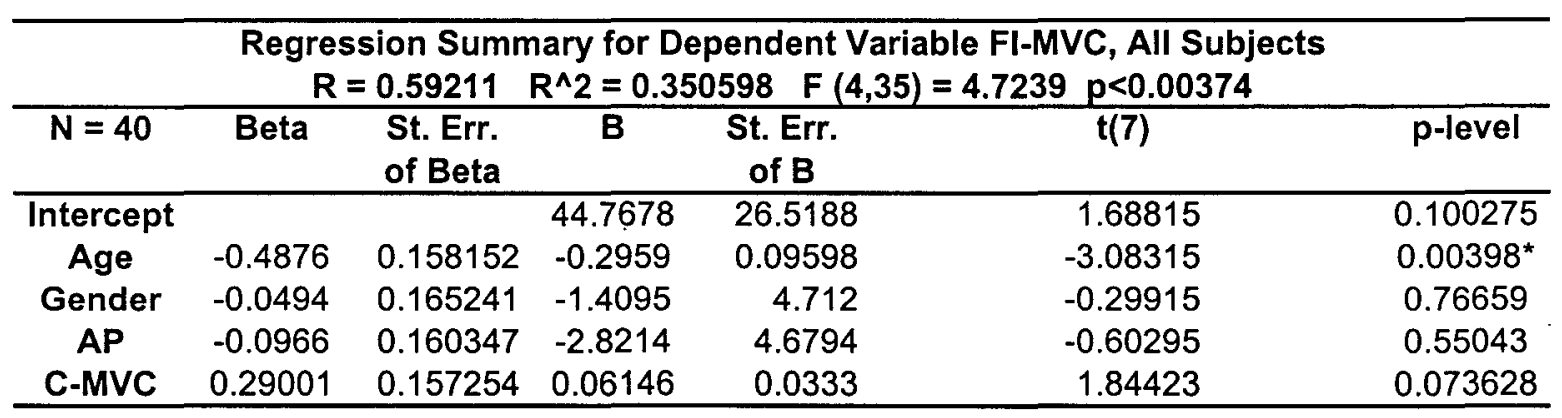

\begin{tabular}{ccccccc}
\hline \multicolumn{7}{c}{ Estimated Adductor Pollicis Muscle Length - All Subjects } \\
\hline Effect & df & MS & df & MS & Fge & 2 Gender \\
& Effect & Effect & Error & Error & p-level \\
\hline 1 & 1 & 1.5408 & 44 & 0.17125 & 8.9976 & $0.0044^{*}$ \\
2 & 1 & 1.08 & 44 & 0.17125 & 6.3066 & $0.0157^{*}$ \\
$1 \times 2$ & 1 & 0.0208 & 44 & 0.17125 & 0.12165 & 0.7289 \\
\hline
\end{tabular}


Appendix D

Raw Data 


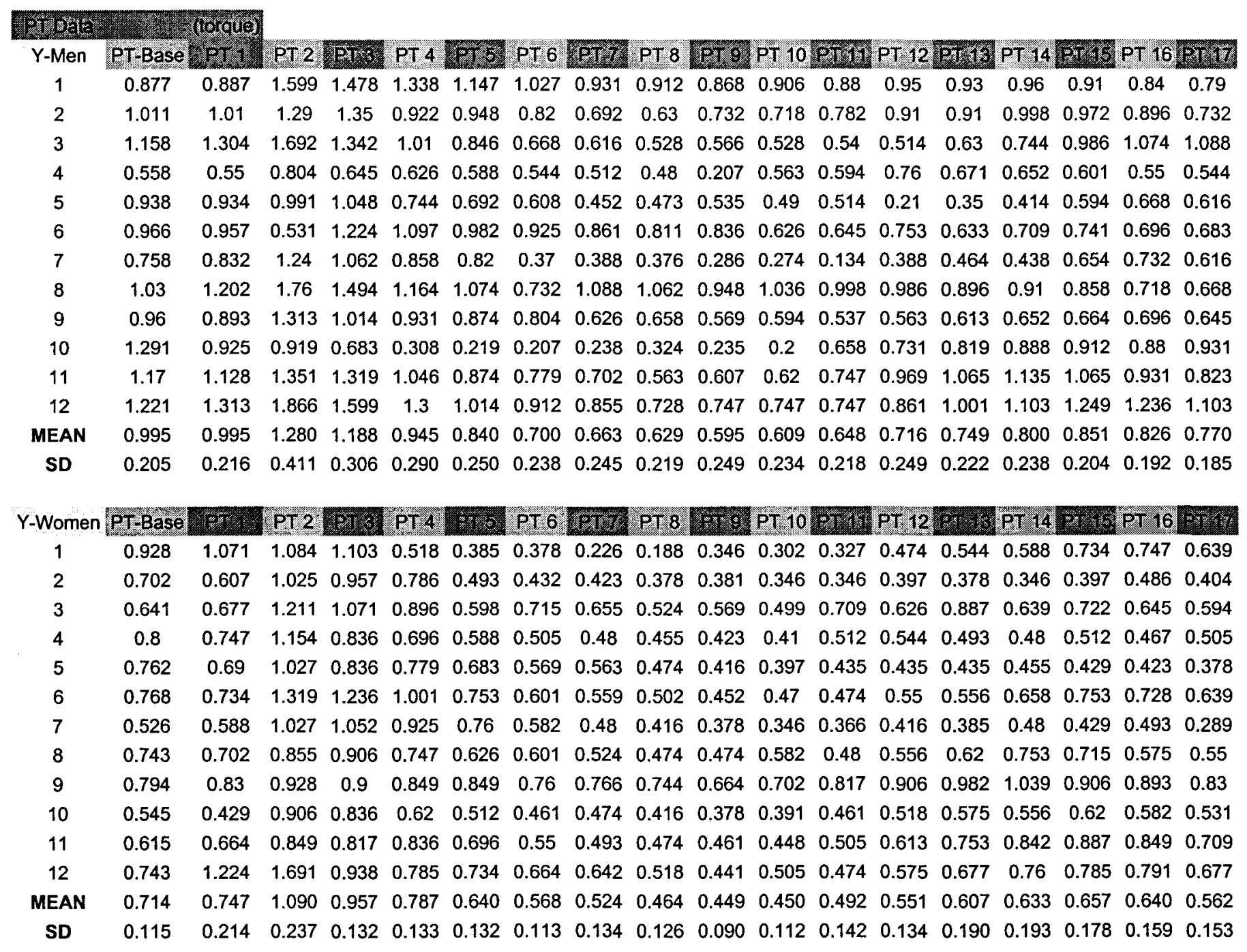




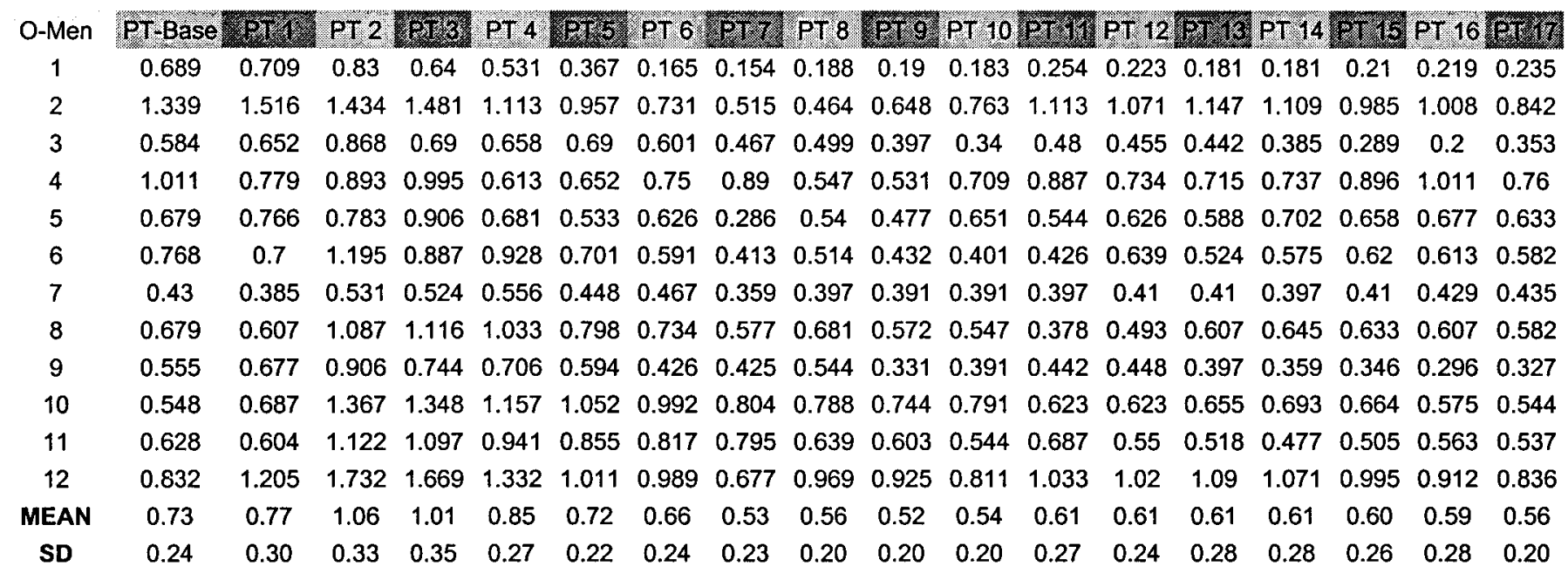

O-Women PT-Base GRT $\begin{array}{llllllllllllllllll}0.392 & 0.435 & 0.387 & 0.42 & 0.309 & 0.404 & 0.677 & 0.504 & 0.513 & 0.674 & 0.642 & 0.534 & 0.54 & 0.496 & 0.451 & 0.512 & 0.448 & 0.385\end{array}$ $\begin{array}{lllllllllllllllllll}0.523 & 0.594 & 0.639 & 0.518 & 0.385 & 0.391 & 0.353 & 0.353 & 0.391 & 0.369 & 0.386 & 0.461 & 0.617 & 0.642 & 0.648 & 0.677 & 0.62 & 0.648\end{array}$ $\begin{array}{lllllllllllllllllll}0.501 & 0.407 & 0.443 & 0.372 & 0.342 & 0.452 & 0.4 & 0.324 & 0.305 & 0.29 & 0.306 & 0.256 & 0.205 & 0.246 & 0.246 & 0.284 & 0.361 & 0.396\end{array}$ $\begin{array}{llllllllllllllllll}0.383 & 0.372 & 0.41 & 0.366 & 0.328 & 0.381 & 0.335 & 0.28 & 0.251 & 0.268 & 0.259 & 0.391 & 0.442 & 0.451 & 0.455 & 0.451 & 0.372 & 0.404\end{array}$ $\begin{array}{lllllllllllllllllll}0.552 & 0.722 & 1.044 & 0.763 & 0.594 & 0.445 & 0.433 & 0.292 & 0.306 & 0.295 & 0.258 & 0.378 & 0.416 & 0.461 & 0.477 & 0.458 & 0.461 & 0.439\end{array}$ $\begin{array}{llllllllllllllllllll}0.446 & 0.423 & 0.427 & 0.401 & 0.361 & 0.362 & 0.429 & 0.356 & 0.379 & 0.351 & 0.372 & 0.426 & 0.267 & 0.305 & 0.331 & 0.369 & 0.416 & 0.261\end{array}$ $\begin{array}{llllllllllllllllllll}0.513 & 0.362 & 0.483 & 0.547 & 0.521 & 0.477 & 0.407 & 0.391 & 0.366 & 0.334 & 0.41 & 0.413 & 0.385 & 0.404 & 0.42 & 0.356 & 0.362 & 0.35\end{array}$ $\begin{array}{llllllllllllllllll}0.446 & 0.499 & 0.429 & 0.477 & 0.607 & 0.537 & 0.496 & 0.27 & 0.296 & 0.397 & 0.394 & 0.321 & 0.388 & 0.394 & 0.394 & 0.394 & 0.353 & 0.327\end{array}$ $\begin{array}{lllllllllllllllllllll}0.482 & 0.524 & 0.48 & 0.601 & 0.595 & 0.589 & 0.596 & 0.484 & 0.459 & 0.389 & 0.392 & 0.403 & 0.528 & 0.613 & 0.575 & 0.509 & 0.553 & 0.512\end{array}$ $\begin{array}{lllllllllllllllllll}0.37 & 0.383 & 0.435 & 0.48 & 0.449 & 0.442 & 0.429 & 0.416 & 0.461 & 0.448 & 0.582 & 0.149 & 0.302 & 0.372 & 0.404 & 0.474 & 0.442 & 0.474\end{array}$ $\begin{array}{llllllllllllllllll}0.564 & 0.575 & 0.499 & 0.423 & 0.518 & 0.461 & 0.505 & 0.395 & 0.585 & 0.398 & 0.436 & 0.55 & 0.645 & 0.588 & 0.563 & 0.569 & 0.55 & 0.563\end{array}$

$\begin{array}{cllllllllllllllllll}12 & 0.533 & 0.645 & 0.711 & 0.688 & 0.596 & 0.551 & 0.576 & 0.418 & 0.449 & 0.401 & 0.379 & 0.493 & 0.474 & 0.442 & 0.499 & 0.544 & 0.531 & 0.556 \\ \text { MEAN } & 0.475 & 0.495 & 0.532 & 0.505 & 0.467 & 0.458 & 0.470 & 0.374 & 0.397 & 0.385 & 0.401 & 0.398 & 0.434 & 0.451 & 0.455 & 0.466 & 0.456 & 0.443\end{array}$ $\begin{array}{lllllllllllllllllll}\text { SD } & 0.067 & 0.118 & 0.187 & 0.126 & 0.118 & 0.071 & 0.103 & 0.076 & 0.100 & 0.106 & 0.114 & 0.114 & 0.136 & 0.120 & 0.110 & 0.107 & 0.090 & 0.112\end{array}$ 


\begin{tabular}{|c|c|c|c|c|c|c|c|c|c|c|c|c|}
\hline & & a & & & & vats & Vo & Tive & & & & \\
\hline & & & & & & & & & & & & \\
\hline 1 & & 6.46 & 6.33 & 5.32 & 5.53 & 5.27 & 4.28 & 27 & 5.45 & $9 r$ & 3 & \\
\hline 2 & 12.76 & 11.58 & 11.56 & 11.37 & 10.34 & 10.88 & 9.21 & 8.73 & 7.71 & 8.63 & 8.4 & 13.71 \\
\hline 3 & 14.03 & 13.7 & 10.45 & 10.31 & 10.09 & 9.42 & 8.58 & 7.52 & 7.65 & 6.24 & 5.14 & 11.36 \\
\hline 4 & 8.4 & 8.73 & 6.98 & 7.1 & 7.08 & 5.72 & 5.92 & 5.83 & 5.67 & 5.27 & 5.4 & 7.81 \\
\hline 5 & 11.26 & 12.71 & 12.68 & 11.11 & 8.21 & 7.66 & 8.28 & 8.02 & 6.44 & 7.29 & 6.48 & 12.58 \\
\hline 6 & 5.32 & 4.21 & 4.29 & 2.87 & 3.13 & 3.02 & 3.02 & 2.46 & 1.44 & 2.27 & 2.59 & 3.687 \\
\hline 7 & 11.43 & 11.98 & 11.04 & 9.96 & 8.69 & 9.81 & 8.84 & 8.39 & 7.51 & 7.19 & 7.27 & 9.02 \\
\hline 8 & 13.17 & 10.47 & 9.67 & 8.19 & 7.53 & 5.32 & 6.15 & 7.51 & 5.66 & 5.04 & 6.59 & 6.54 \\
\hline 9 & 8.88 & 6.88 & 7.28 & 6.13 & 6.04 & 5.88 & 5.13 & 5.34 & 6.35 & 6.56 & 5.82 & 8.56 \\
\hline 10 & 9.53 & 9.33 & 8.26 & 8.16 & 7.1 & 6.36 & 5.04 & 4.2 & 3.44 & 4.53 & 5.08 & 7.92 \\
\hline 11 & 7.9 & 7.6 & 6.75 & 6.03 & 5.78 & 5.32 & 5.14 & 4.81 & 4.47 & 4.45 & 4.29 & 6.72 \\
\hline 12 & 10.65 & 10.01 & 7.92 & 8.4 & 6.7 & 5.89 & 5.61 & 5.13 & 4.7 & 4.17 & 4.19 & 8.73 \\
\hline MEAN & 10.016 & 9.472 & 8.601 & 7.913 & 7.185 & 6.713 & 6.267 & 6.101 & 5.541 & 5.551 & 5.473 & 8.601 \\
\hline SD & 2.666 & 2.827 & 2.487 & 2.559 & 2.012 & 2.282 & 1.996 & 1.922 & 1.849 & 1.713 & 1.564 & 2.810 \\
\hline
\end{tabular}

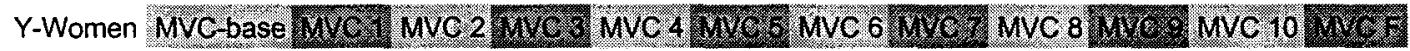

$\begin{array}{ccccccccccccc}1 & 8.36 & 7.67 & 7.56 & 6.48 & 5.67 & 6.31 & 4.62 & 4.4 & 3.83 & 3.46 & 3.42 & 5.74 \\ 2 & 5.27 & 5.17 & 4.31 & 3.74 & 4.91 & 4.23 & 3.83 & 5.08 & 4.71 & 4.09 & 4.12 & 7.18 \\ 3 & 7.17 & 7.24 & 5.95 & 5.42 & 4.71 & 3.48 & 3.94 & 3.5 & 4.34 & 4.68 & 4.27 & 5.74 \\ 4 & 6.51 & 6.18 & 5.02 & 4.92 & 4.71 & 4.7 & 4.53 & 3.85 & 3.81 & 3.84 & 4.42 & 4.88 \\ 5 & 7.63 & 7.68 & 6.84 & 6.17 & 5.25 & 4.72 & 5.13 & 4.85 & 4.8 & 4.63 & 4.74 & 6.15 \\ 6 & 7.28 & 7.24 & 5.81 & 5.2 & 5.12 & 4.98 & 4.14 & 3.63 & 3.27 & 3.18 & 3.12 & 5.82 \\ 7 & 7.08 & 7.01 & 5.78 & 5.77 & 6.28 & 6.05 & 4.22 & 4.6 & 5.43 & 4.53 & 3.84 & 7.65 \\ 8 & 7.24 & 6.77 & 7.6 & 6.3 & 7.09 & 6.59 & 6.38 & 6.14 & 6.06 & 6.03 & 6.23 & 5.89 \\ 9 & 5.58 & 4.87 & 4.67 & 4.11 & 3.89 & 3.65 & 3.85 & 3.51 & 3.59 & 2.77 & 2.66 & 3.94 \\ 10 & 5.28 & 4.89 & 5.1 & 5.08 & 4.57 & 4.17 & 4.18 & 3.62 & 3.45 & 3.49 & 3.81 & 5.41 \\ 11 & 4.93 & 4.44 & 3.74 & 4.28 & 4.64 & 4.33 & 3.7 & 3.97 & 4.51 & 4.27 & 3.58 & 4.15 \\ 12 & 6.32 & 5.81 & 3.89 & 4.33 & 4.53 & 3.77 & 4.58 & 5.01 & 4.51 & 4.24 & 3.96 & 4.59 \\ \text { MEAN } & 6.554 & 6.248 & 5.523 & 5.150 & 5.114 & 4.748 & 4.425 & 4.347 & 4.359 & 4.101 & 4.014 & 5.595 \\ \text { SD } & 1.087 & 1.178 & 1.314 & 0.912 & 0.870 & 1.050 & 0.740 & 0.818 & 0.831 & 0.855 & 0.901 & 1.112\end{array}$




\begin{tabular}{|c|c|c|c|c|c|c|c|c|c|c|c|c|}
\hline Den & Vo-base & Mus: & Mve 2 & 1.c3: & Mve & Bues: & MVC & Ye & Kav & 2e: & MVC 10 & Net \\
\hline 1 & 8.85 & 8.12 & 7.57 & 7.57 & 6.63 & 6.16 & 6.42 & 5.72 & 5.85 & 5.94 & 5.92 & 6.87 \\
\hline 2 & 7.26 & 7.6 & 6.69 & 5.57 & 5.29 & 5.35 & 5.93 & 6.05 & 5 & 5.21 & 5.12 & 6.87 \\
\hline 3 & 6.81 & 5.96 & 4.85 & 4.81 & 4.24 & 5.28 & 4.43 & 4.89 & 4.7 & 4.88 & 4.5 & 7.26 \\
\hline 4 & 6.47 & 6.02 & 5.3 & 5.62 & 5.05 & 5.74 & 5.32 & 5.12 & 4.72 & 5.55 & 78 & 6.37 \\
\hline 5 & 7.38 & 6.48 & 6.01 & 7.94 & 8.55 & 7.45 & 7.74 & 8.23 & 8.53 & 8.98 & 7.65 & 7.84 \\
\hline 6 & 6.39 & 4.82 & 5.4 & 5.13 & 5.21 & 5.04 & 4.59 & 4.28 & 4.12 & 3.7 & 4.09 & 5.42 \\
\hline 7 & 4.04 & 4.54 & 4.41 & 3.55 & 4.45 & 3.89 & 3.85 & 3.95 & 34 & 3.18 & 3.5 & 3.71 \\
\hline 8 & 8.52 & 7.05 & 7.27 & 6.53 & 6.65 & 6.96 & 6.94 & 7.25 & 69 & 7.01 & 8.03 & 8.13 \\
\hline 9 & 6.1 & 5.27 & 5.76 & 5.7 & 5.79 & 5.78 & 6 & 5.16 & 5.21 & 5.56 & 5.42 & 7.16 \\
\hline 10 & 5.36 & 5.37 & 5.57 & 5.01 & 4.57 & 5.23 & 4.57 & 4.99 & 5.29 & 5.12 & 4.62 & 4.79 \\
\hline 11 & 6.18 & 5.46 & 4.67 & 4.79 & 4.37 & 4.78 & 4.76 & 4.32 & 4.1 & 3.66 & 4.54 & 5.8 \\
\hline 12 & 8.54 & 9.53 & 9.7 & 9.8 & 8.2 & 7.33 & 7.96 & 6.94 & 5.7 & 7.58 & 6.98 & 8.18 \\
\hline MEAN & 6.8250 & 6.3517 & 6.1000 & 6.0017 & 5.7500 & 5.7492 & 5.7092 & 5.5750 & 5.2708 & 5.5308 & 5.5125 & 6.5333 \\
\hline SD & 1.4004 & 1.4805 & 1.5026 & 1.7053 & 1.4675 & 1.0692 & 1.3497 & 1.3159 & 1.3580 & 1.6929 & 1.4239 & 1.3794 \\
\hline
\end{tabular}

\begin{tabular}{|c|c|c|c|c|c|c|c|c|c|c|c|c|}
\hline & ve-uas & nued & 20 & Mve. & me 4 & Wes & MVC 6 & ave & Mve 8 & 14ro & uve 10 & nve \\
\hline 1 & 7.75 & 5.43 & 5.65 & 4.99 & 4.72 & 4.59 & 5.67 & 5.18 & 5.31 & 5.84 & 5.95 & 7.03 \\
\hline 2 & 6.65 & 6.07 & 5.55 & 5.18 & 4.98 & 4.96 & 4.74 & 4.63 & 4.92 & 5.04 & .98 & 6.21 \\
\hline 3 & 5.37 & 6.39 & 6.25 & 5.38 & 5.74 & 5.11 & 5.58 & 4.99 & 5.28 & 5.39 & .03 & 5.57 \\
\hline 4 & 3.63 & 3.03 & 3.5 & 3.47 & 3.52 & 3.66 & 3.52 & 3.83 & 3.8 & 3.97 & .21 & 3.46 \\
\hline 5 & 75 & 7.25 & 6.71 & 5.66 & 5.37 & 5.03 & 4.37 & 4.51 & 4.8 & 3.87 & .13 & 6.51 \\
\hline 6 & 27 & 5.62 & 4.66 & 5.13 & 5.56 & 4.65 & 4.72 & 4.44 & 5.12 & 5.14 & 4.49 & 5.5 \\
\hline 7 & 3.09 & 4.43 & 3.34 & 4.07 & 3.96 & 3.97 & 3.64 & 4.23 & 4 & 4.08 & .97 & 3.29 \\
\hline 8 & 65 & 3.73 & & 3.38 & 3.82 & 3.67 & 3.36 & 3.2 & 3.2 & 3.4 & 3.6 & 3.7 \\
\hline 9 & 67 & 6.81 & 6.83 & 5.95 & 5.46 & 6.24 & 5.56 & 5.05 & 6.03 & 5.4 & 5.85 & 5.52 \\
\hline 10 & 5.02 & 4.82 & 5.39 & 5.25 & 4.32 & 4.84 & 4.34 & 5.59 & 4.98 & 4.17 & 4.25 & 6.04 \\
\hline 11 & & 6.14 & 7 & 7.24 & 6.36 & 6.21 & 6.21 & 5.64 & 4.84 & 5.34 & 5.83 & 6.71 \\
\hline 12 & & 4.35 & 4.03 & 3.41 & 3.16 & 2.95 & 3.42 & 2.74 & 2.91 & 2.68 & 2.77 & 4.53 \\
\hline MEAN & 5.725 & 5.339 & 5.168 & 4.926 & 4.748 & 4.657 & 4.594 & 4.503 & 4.608 & 4.527 & 4.588 & 5.339 \\
\hline$S D$ & 1.456 & 1.286 & 1.416 & 1.164 & 0.995 & 0.988 & 0.990 & 0.896 & 0.921 & 0.967 & 0.976 & 1.298 \\
\hline
\end{tabular}




\begin{tabular}{|c|c|c|c|c|c|c|c|c|c|c|c|}
\hline Men & HRT & te & T 2 & ART 3] & HRT 4 & KRT 5 & HRT & GRT: & HRT 8 & GRS: & HRT 10 \\
\hline 1 & 0.08 & 0.0857 & 0.0813 & 0.0865 & 0.0921 & 0.0842 & 0.0891 & 0.0828 & 0.0805 & 0.0756 & 0.0794 \\
\hline 2 & 0.0795 & 0.0798 & 0.064 & 0.0726 & 0.0828 & 0.094 & 0.0914 & 0.0798 & 0.0768 & 0.0708 & 0.0674 \\
\hline 3 & 0.0712 & 0.0693 & 0.0363 & 0.0768 & 0.0783 & 0.1003 & 0.1236 & 0.1045 & 0.1078 & 0.094 & 0.112 \\
\hline 4 & 075 & 0.0633 & 0.082 & 0.07 & 0.073 & 0.0711 & 0.0696 & 0.0678 & 0.0614 & 0.0652 & 0.0618 \\
\hline 5 & 0.0746 & 0.0756 & 0.0775 & 0.0798 & 0.0929 & 0.0977 & 0.0805 & 0.0704 & 0.0798 & 0.0734 & 0.0734 \\
\hline 6 & 0.0679 & 0.0745 & 0.0468 & 0.0749 & 0.0734 & 0.0749 & 0.079 & 0.0678 & 0.0509 & 0.0741 & 0.0625 \\
\hline 7 & 0.0586 & 0.0618 & 0.0745 & 0.0794 & 0.0857 & 0.0734 & 0.0401 & 0.0865 & 0.0959 & 0.0588 & 0.0929 \\
\hline 8 & 0709 & 0.067 & 0.0588 & 0.055 & 0.058 & 0.0505 & 0.0352 & 0.0517 & 0.0509 & 0.0434 & 0.0505 \\
\hline 9 & 0.0662 & 0.079 & 0.0846 & 0.0951 & 0.0764 & 0.1041 & 0.0813 & 0.1056 & 0.1179 & 0.0962 & 0.1003 \\
\hline 10 & 0.0493 & 0.0693 & 0.0899 & 0.1026 & 0.0989 & 0.1389 & 0.0704 & 0.1137 & 0.1573 & 0.0333 & 0.0434 \\
\hline 11 & 0.0792 & 0.0872 & 0.0936 & 0.0902 & 0.1003 & 0.085 & 0.0854 & 0.0738 & 0.073 & 0.0846 & 0.0876 \\
\hline 12 & 0.0746 & 0.0786 & 0.0726 & 0.0599 & 0.0771 & 0.0726 & 0.061 & 0.0685 & 0.0689 & 0.0685 & 0.0738 \\
\hline MEAN & 0.0706 & 0.0743 & 0.0718 & 0.0786 & 0.0824 & 0.0872 & 0.0756 & 0.0811 & 0.0851 & 0.0698 & 0.0754 \\
\hline SD & 0.0091 & 0.0083 & 0.0174 & 0.0138 & 0.0123 & 0.0223 & 0.0235 & 0.0186 & 0.0307 & 0.0184 & 0.0202 \\
\hline
\end{tabular}

\begin{tabular}{ccccccccccccc} 
Y-women & HRT-Base & HRT & HRT 2 & HRT & HRT 4 & HRT 5 & HRT 6 & HRT & HRT 8 & HRT & HRT & HR \\
\hline 1 & 0.0969 & 0.1067 & 0.085 & 0.0887 & 0.0786 & 0.0749 & 0.1281 & 0.058 & 0.0625 & 0.1254 & 0.0505 \\
2 & 0.1075 & 0.0989 & 0.1801 & 0.1651 & 0.1981 & 0.1861 & 0.1625 & 0.1194 & 0.1251 & 0.0696 & 0.1003 \\
3 & 0.0646 & 0.0813 & 0.0865 & 0.0899 & 0.0794 & 0.0502 & 0.061 & 0.0738 & 0.0842 & 0.0693 & 0.0947 \\
4 & 0.0819 & 0.0831 & 0.0708 & 0.082 & 0.0824 & 0.0622 & 0.0588 & 0.0633 & 0.0494 & 0.0588 & 0.0517 \\
5 & 0.0659 & 0.0805 & 0.0734 & 0.0749 & 0.0887 & 0.0857 & 0.0865 & 0.079 & 0.082 & 0.0846 & 0.0738 \\
6 & 0.0669 & 0.0872 & 0.0857 & 0.0876 & 0.097 & 0.0951 & 0.1015 & 0.1082 & 0.0857 & 0.0865 & 0.0813 \\
7 & 0.0719 & 0.0618 & 0.0674 & 0.0865 & 0.0734 & 0.0794 & 0.0914 & 0.1048 & 0.0835 & 0.097 & 0.0809 \\
8 & 0.0612 & 0.0786 & 0.0794 & 0.0637 & 0.0734 & 0.0655 & 0.0783 & 0.0678 & 0.0674 & 0.0584 & 0.0547 \\
9 & 0.1085 & 0.103 & 0.1062 & 0.0902 & 0.082 & 0.073 & 0.055 & 0.0678 & 0.0693 & 0.0517 & 0.0614 \\
10 & 0.0666 & 0.0603 & 0.061 & 0.073 & 0.0831 & 0.0775 & 0.0771 & 0.0738 & 0.0678 & 0.0648 & 0.0625 \\
11 & 0.0722 & 0.1077 & 0.091 & 0.1412 & 0.0962 & 0.1003 & 0.0899 & 0.0816 & 0.0835 & 0.0798 & 0.085 \\
12 & 0.0662 & 0.0633 & 0.0652 & 0.0622 & 0.0798 & 0.0655 & 0.061 & 0.0539 & 0.061 & 0.0652 & 0.0719 \\
MEAN & 0.078 & 0.084 & 0.088 & 0.092 & 0.093 & 0.085 & 0.088 & 0.079 & 0.077 & 0.076 & 0.072 \\
SD & 0.017 & 0.017 & 0.032 & 0.031 & 0.034 & 0.035 & 0.032 & 0.021 & 0.019 & 0.020 & 0.017
\end{tabular}




\begin{tabular}{cccccccccccccc}
\hline O-Men & HRT-Base & HRT & HRT 2 & HRT & HRT 4 & HRT & HRT 6 & HRT & HRT 8 & HRT & HRT & 10 \\
1 & 0.0699 & 0.0876 & 0.0625 & 0.052 & 0.0607 & 0.0592 & 0.0694 & 0.0631 & 0.0499 & 0.0629 & 0.0721 \\
2 & 0.0749 & 0.0708 & 0.0674 & 0.0745 & 0.073 & 0.0831 & 0.0816 & 0.0854 & 0.0914 & 0.094 & 0.0944 \\
3 & 0.0553 & 0.0685 & 0.067 & 0.0685 & 0.079 & 0.0771 & 0.0674 & 0.0719 & 0.0696 & 0.0625 & 0.0513 \\
4 & 0.0716 & 0.0839 & 0.0783 & 0.0745 & 0.0404 & 0.0505 & 0.0404 & 0.0629 & 0.0359 & 0.0412 & 0.0786 \\
5 & 0.0899 & 0.1007 & 0.1077 & 0.1015 & 0.0828 & 0.0829 & 0.1618 & 0.322 & 0.1296 & 0.067 & 0.084 \\
6 & 0.0699 & 0.064 & 0.0947 & 0.0959 & 0.1228 & 0.1513 & 0.163 & 0.1127 & 0.173 & 0.1239 & 0.079 \\
7 & 0.0749 & 0.0944 & 0.091 & 0.1082 & 0.1213 & 0.1367 & 0.134 & 0.1393 & 0.1179 & 0.1 & 0.112 \\
8 & 0.0666 & 0.0614 & 0.0734 & 0.0959 & 0.0977 & 0.0771 & 0.0813 & 0.0824 & 0.0839 & 0.0786 & 0.0947 \\
9 & 0.0549 & 0.0603 & 0.0524 & 0.0592 & 0.0678 & 0.0532 & 0.0674 & 0.0592 & 0.0625 & 0.0689 & 0.0554 \\
10 & 0.0682 & 0.0711 & 0.0738 & 0.0805 & 0.0842 & 0.0835 & 0.0962 & 0.0861 & 0.1 & 0.0887 & 0.0846 \\
11 & 0.0533 & 0.0476 & 0.0487 & 0.0914 & 0.0932 & 0.0809 & 0.0813 & 0.0734 & 0.0689 & 0.0614 & 0.0794 \\
12 & 0.0562 & 0.0464 & 0.0577 & 0.0696 & 0.0667 & 0.0547 & 0.0865 & 0.0786 & 0.0678 & 0.0741 & 0.0711 \\
MEAN & 0.0671 & 0.0714 & 0.0729 & 0.0810 & 0.0825 & 0.0825 & 0.0942 & 0.1031 & 0.0875 & 0.0769 & 0.0797 \\
SD & 0.0108 & 0.0173 & 0.0177 & 0.0176 & 0.0239 & 0.0315 & 0.0386 & 0.0726 & 0.0381 & 0.0219 & 0.0167
\end{tabular}

\begin{tabular}{|c|c|c|c|c|c|c|c|c|c|c|c|}
\hline$m$ & M-Da & 12 & HRT 2 & $12 \pi$ & IRT & HET & HRT 6 & $2+24$ & I HRT 8 & 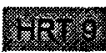 & HRT 10 \\
\hline 1 & 0.0699 & 0.0607 & 0.0505 & 0.0389 & 0.0303 & 0.0367 & 0.0562 & 0.0408 & $0.043 \varepsilon$ & 0.0505 & 0.05 \\
\hline 2 & .0999 & 0884 & 0.0779 & 0.0812 & 0.0595 & 0.0925 & 0.1075 & 0.1074 & 0.0925 & 0.0997 & 0.086 \\
\hline 3 & 6 & 629 & 6683 & 404 & 0423 & 0.0464 & 0412 & 513 & 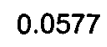 & 0554 & .05 \\
\hline 4 & 1248 & .0984 & 0917 & .1498 & 1011 & 0.0964 & 0.1078 & 0.0944 & 0.0987 & 0.1086 & 0.168 \\
\hline 5 & .0749 & 0.0914 & 0.0816 & 0.0899 & 0.0801 & 0.073 & 0.0824 & 0.0779 & 0.0927 & 0.0738 & 0.091 \\
\hline 6 & 0832 & 0.097 & 0.0794 & .0864 & 0.064 & 0.1056 & 0.1015 & 0.1135 & 0.058 & 0.0498 & 0.07 \\
\hline 7 & .0699 & 0.0622 & .0532 & .0573 & 0.0618 & 0.061 & 0.0577 & 0.0629 & 0.0704 & 0.067 & .065 \\
\hline 8 & 0.0782 & 0.0734 & 0.0681 & 0.0565 & 0.0655 & 0.0711 & 0.0412 & 0.0569 & 0.0685 & 0.0951 & 0.0625 \\
\hline 9 & 0.0849 & 0.0764 & 0.0693 & 0.0989 & 0.0784 & 0.1071 & 0.0798 & 0.0821 & 0.0927 & 0.0799 & 0.0846 \\
\hline 10 & 0.0589 & 0.0513 & 1408 & 0.1865 & .0946 & 0.0461 & 0.1168 & 0.073 & 0.0618 & 0.0547 & 0.0629 \\
\hline 1 & 0.0726 & 0.0685 & 0891 & .0768 & .0786 & 0.0934 & 0.0979 & 0.0333 & 0.0614 & 0.0895 & 0.0846 \\
\hline 2 & 0.0722 & 0.0846 & .0817 & 0.0719 & 0.0611 & 0.0722 & 0.0549 & 0.0739 & 0.0647 & 0.0502 & 0.0723 \\
\hline$m a r$ & 0.0801 & 0.0763 & 0793 & .0862 & .0681 & 0.0751 & 787 & 0.0723 & 0.071 & 0.0729 & .08 \\
\hline SD & 0.0173 & 156 & & 33 & & & & & & & \\
\hline
\end{tabular}




\begin{tabular}{|c|c|c|c|c|c|c|c|c|c|c|c|}
\hline \multicolumn{12}{|c|}{ MRTD } \\
\hline Y-Men & MRTD-B & Whror & MRTD 2 & MRTO 3 & MRTD 4 & 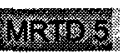 & MRTD 6 & Whero: & MRTD 8 & MRTD & MRTD 10 \\
\hline 1 & 22.75 & 29.19 & 42.88 & 33.59 & 30.52 & 27.05 & 26.31 & 23.71 & 24.84 & 23.78 & 23.71 \\
\hline 2 & 26.28 & 24.44 & 36.6 & 39.28 & 26.04 & 26.04 & 22.3 & 19.24 & 18.44 & 18.96 & 20.04 \\
\hline 3 & 33.1 & 37.6 & 48 & 35.4 & 26.8 & 21.2 & 18 & 17.2 & 14.4 & 14.8 & 15 \\
\hline 4 & 16.38 & 16.4 & 21.8 & 19 & 21.4 & 19.8 & 18.6 & 17.9 & 18.4 & 16.6 & 18.5 \\
\hline 5 & 21.97 & 22.2 & 24 & 25.8 & 16.6 & 9.8 & 10.9 & 12 & 12.4 & 12.8 & 13.2 \\
\hline 6 & 22.47 & 23.57 & 14.51 & 31.76 & 25.86 & 24.69 & 22.75 & 21.94 & 21.28 & 18.38 & 16.8 \\
\hline 7 & 18.88 & 20.2 & 32.2 & 25.2 & 22.4 & 22.2 & 14 & 7.2 & 9.2 & 6.8 & 6.6 \\
\hline 8 & 26.94 & 33.7 & 52.12 & 46.94 & 37.66 & 33.5 & 31.56 & 29.02 & 28.82 & 28.2 & 29.12 \\
\hline 9 & 27.46 & 22.7 & 31.7 & 23 & 22 & 18.2 & 16.3 & 13.9 & 13.9 & 10.9 & 12.8 \\
\hline 10 & 32.67 & 27.3 & 27.5 & 17.3 & 5.8 & 3.7 & 5.5 & 5.7 & 7.4 & 4.9 & 4.5 \\
\hline 11 & 29.54 & 26.5 & 36.1 & 31.2 & 23.6 & 21.7 & 19.8 & 17.7 & 15.5 & 16.8 & 17 \\
\hline 12 & 31.37 & 31.9 & 50.5 & 36.9 & 34.5 & 28.1 & 25.1 & 21.9 & 22.1 & 23.3 & 21.8 \\
\hline MEAN & 25.82 & 26.31 & 34.83 & 30.45 & 24.43 & 21.33 & 19.26 & 17.28 & 17.22 & 16.35 & 16.59 \\
\hline SD & 5.41 & 6.02 & 11.90 & 8.72 & 8.27 & 8.07 & 7.10 & 6.75 & 6.34 & 6.89 & 6.91 \\
\hline Y-Women & MRTD-B & URTD & MRTD 2 & WRTO: & MRTD 4 & MrTo 5 & MRTD 6 & MRTD & MRTD 8 & MRTO & MRTD 10 \\
\hline 1 & 21.11 & 25.3 & 25.9 & 23 & 12 & 10.2 & 7.7 & 7 & 4.5 & 4.4 & 7.2 \\
\hline 2 & 16.12 & 14.7 & 27.46 & 25.2 & 21.11 & 10.1 & 11.8 & 13.1 & 14.4 & 11.5 & 11.7 \\
\hline 3 & 16.1 & 16.2 & 28.5 & 27.8 & 25.5 & 17.6 & 18.8 & 18.6 & 15.7 & 16.2 & 13.1 \\
\hline 4 & 24.87 & 23.7 & 34.5 & 25.8 & 21.4 & 17.9 & 16.5 & 14.8 & 15.8 & 13.7 & 12.3 \\
\hline 5 & 21.75 & 19.1 & 32.4 & 26 & 22.5 & 21.4 & 17.3 & 17.1 & 14.8 & 13.6 & 12.4 \\
\hline 6 & 19.63 & 18.9 & 36.1 & 32.1 & 25.8 & 21.8 & 17.9 & 16.2 & 14.5 & 14.2 & 14.3 \\
\hline 7 & 15.06 & 13.8 & 26.3 & 24.2 & 24.6 & 20.4 & 16 & 11.6 & 8.6 & 9 & 9.3 \\
\hline 8 & 20.72 & 17.4 & 23.8 & 26.8 & 21.3 & 18.3 & 18.9 & 16 & 16.2 & 15.8 & 21.2 \\
\hline 9 & 18.49 & 17.92 & 24.08 & 22.24 & 18.88 & 18.12 & 17.15 & 17.87 & 18.27 & 18.32 & 17.66 \\
\hline 10 & 15.82 & 11.8 & 23.7 & 21 & 17.3 & 14.2 & 13.1 & 11.6 & 12 & 11.9 & 11.2 \\
\hline 11 & 14.93 & 16.95 & 18.48 & 19.44 & 20.21 & 17.15 & 14.05 & 12.78 & 12.06 & 12.11 & 10.49 \\
\hline 12 & 20.09 & 32.8 & 47.3 & 28.9 & 22.7 & 25.3 & 20.6 & 20.5 & 16.9 & 15.8 & 14.9 \\
\hline MEAN & 18.724 & 19.048 & 29.043 & 25.207 & 21.108 & 17.706 & 15.817 & 14.763 & 13.644 & 13.044 & 12.979 \\
\hline SD & 3.152 & 5.763 & 7.608 & 3.530 & 3.830 & 4.499 & 3.601 & 3.733 & 3.865 & 3.701 & 3.738 \\
\hline
\end{tabular}




\begin{tabular}{|c|c|c|c|c|c|c|c|c|c|c|c|}
\hline & & & & & & & & magnang & & In & \\
\hline 1 & 18.65 & 17.7 & 15.7 & 11.5 & 13.6 & 6.9 & 5.2 & 3 & 4.2 & 4.7 & 4.9 \\
\hline 2 & 38.66 & 37.3 & 31.7 & 46.4 & 19.9 & 21.9 & 20.5 & 13.9 & 16.7 & 12.1 & 14.5 \\
\hline 3 & 16.6 & 18.68 & 22.4 & 17.66 & 15.73 & 16.85 & 17.92 & 13.44 & 15.47 & 11.2 & 12.37 \\
\hline 4 & 24.58 & 18.4 & 14.8 & 26 & 16.1 & 16.9 & 14.4 & 22.9 & 13.7 & 16.8 & 19.1 \\
\hline 5 & 19.49 & 16.6 & 15.5 & 22.3 & 16.2 & 15.1 & 16.7 & 11.9 & 8.4 & 13 & 10.8 \\
\hline 6 & 22.19 & 17.5 & 33.7 & 23.1 & 23.5 & 18.4 & 15.6 & 10.4 & 14.8 & 15.3 & 11.1 \\
\hline 7 & 12.86 & 11.25 & 15.47 & 13.39 & 14.1 & 12.57 & 12.67 & 11.71 & 10.64 & 12.01 & 9.16 \\
\hline 8 & 19.19 & 17.5 & 34 & 40.9 & 26.6 & 24.8 & 24.8 & 18.3 & 20. & 17.1 & 13.6 \\
\hline 9 & 16.15 & 21.6 & 32.5 & 23.9 & 23.8 & 21.2 & 15.6 & 14.4 & 15.5 & 11.2 & 11.1 \\
\hline 10 & 19.5 & 20.9 & 44.9 & 41.5 & 37.3 & 30.7 & 27.1 & 21 & 18.2 & 18.8 & 20.6 \\
\hline 11 & 16.56 & 17 & 28.71 & 29.22 & 24.69 & 24.08 & 22.96 & 25.45 & 21.28 & 18.63 & 16.7 \\
\hline 12 & 20.3 & 34.1 & 46.4 & 46.2 & 34.6 & 23 & 28.9 & 21.4 & 29.9 & 28.2 & 21.7 \\
\hline MEAN & 20.394 & 20.711 & 27.982 & 28.506 & 22.177 & 19.367 & 18.529 & 15.650 & 15.808 & 14.920 & 13.803 \\
\hline SD & 6.497 & 7.475 & 11.270 & 12.383 & 7.813 & 6.298 & 6.700 & 6.353 & 6.626 & 5.767 & 4.973 \\
\hline
\end{tabular}

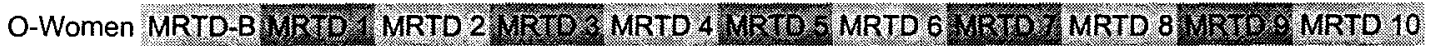

$\begin{array}{cccccccccccc}1 & 9.17 & 8.9 & 10.5 & 15.1 & 10.8 & 13.1 & 18.7 & 13.9 & 14 & 15.6 & 13.8 \\ 2 & 11.82 & 13.6 & 15.5 & 15.34 & 7.9 & 8.27 & 7.8 & 9.2 & 7.4 & 6.61 & 6.74 \\ 3 & 11.52 & 9.1 & 10.36 & 11.6 & 11.8 & 12.1 & 9.6 & 7.2 & 8.1 & 7.52 & 7.49 \\ 4 & 10.28 & 10.84 & 12.65 & 10.59 & 8.9 & 9.42 & 9.72 & 9.24 & 6.16 & 8.91 & 5.8 \\ 5 & 16.41 & 20 & 27.2 & 21.7 & 17.3 & 10.5 & 10.12 & 9.4 & 9.31 & 9 & 7.9 \\ 6 & 10.74 & 9.5 & 11.9 & 11.37 & 9.36 & 6.7 & 10.9 & 10.11 & 10.07 & 9.28 & 9.45 \\ 7 & 13.81 & 8.55 & 12.06 & 14.1 & 10.13 & 11.86 & 10.89 & 11.55 & 10.84 & 9.47 & 11.96 \\ 8 & 12.5 & 12.93 & 12.27 & 14 & 14.91 & 14.05 & 15.37 & 9.32 & 9.11 & 8.81 & 10.43 \\ 9 & 13.15 & 11.6 & 12.1 & 11.5 & 12.7 & 12.3 & 11.8 & 10.6 & 9.7 & 9.3 & 9 \\ 10 & 11.71 & 11.53 & 13.24 & 10 & 8.44 & 17.1 & 17.73 & 14.6 & 15.1 & 14.7 & 16 \\ 11 & 15.22 & 16 & 14.2 & 12 & 13.3 & 12.6 & 10.8 & 10.9 & 15.8 & 8.4 & 12.5 \\ 12 & 15.07 & 16.54 & 19.28 & 17.04 & 13.19 & 12.86 & 12.55 & 9.81 & 9.75 & 7.88 & 6.51 \\ \text { MEAN } & 12.617 & 12.424 & 14.272 & 13.695 & 11.561 & 11.738 & 12.165 & 10.486 & 10.445 & 9.623 & 9.798 \\ \text { SD } & 2.183 & 3.565 & 4.731 & 3.316 & 2.843 & 2.742 & 3.372 & 2.068 & 3.023 & 2.719 & 3.201\end{array}$




\begin{tabular}{|c|c|c|c|c|c|c|c|c|c|c|c|}
\hline Y-Men & $\pi$ & 100: & $\mathrm{mb} 2$ & & mo 4 & & & STS & & & \\
\hline 1 & 20.2 & 056 & 23.461 & 23.203 & 22.664 & 22.162 & 22.033 & 21.171 & 21.107 & 21.416 & 21.325 \\
\hline 2 & 17.762 & 19.036 & 20.322 & 20.014 & 17.275 & 18.11 & 16.412 & 15.473 & 15.345 & 15.602 & 16.245 \\
\hline 3 & 15.528 & 15.238 & 17.454 & 16.669 & 15.82 & 14.405 & 13.968 & 13.583 & 13.029 & 12.566 & 12.464 \\
\hline 4 & 21.095 & 9.859 & 14.032 & 17.712 & 14.65 & 12.296 & 10.792 & 13.068 & 13.642 & 13.008 & 13.114 \\
\hline 5 & 21.286 & 23.088 & 26.87 & 23.14 & 23.165 & 21.814 & 21.519 & 20.747 & 18.92 & 19.615 & 18.419 \\
\hline 6 & 20.214 & 21.146 & 17.84 & 19.821 & 12.966 & 14.187 & 14.342 & 7.756 & 7.512 & 6.933 & 6.599 \\
\hline 7 & 23.623 & 24.13 & 25.235 & 25.39 & 25.712 & 24.683 & 24.554 & 23.756 & 23.306 & 22.754 & 22.779 \\
\hline 8 & 18.541 & 17.467 & 19.666 & 18.097 & 16.708 & 14.598 & 13.878 & 12.296 & 12.104 & 9.441 & 9.981 \\
\hline 9 & 17.634 & 13.21 & 9.197 & 6.264 & 4.167 & 4.052 & 3.782 & 4.373 & 5.44 & 6.881 & 6.792 \\
\hline 10 & 20.532 & 19.962 & 20.76 & 20.541 & 18.612 & 17.801 & 17.57 & 17.441 & 16.811 & 16.786 & 16.593 \\
\hline 11 & 24.031 & 23.461 & 24.85 & 24.58 & 23.217 & 22.689 & 21.531 & 721 & 20.323 & 19.602 & 9.576 \\
\hline MEAN & 20.041 & 19.787 & 19.972 & 19.585 & 17.723 & 16.982 & 16.398 & 15.490 & 15.231 & 14.964 & 14.899 \\
\hline SD & 2.556 & 3.415 & 5.238 & 5.265 & 6.079 & 5.944 & 5.994 & 6.021 & 5.607 & 5.649 & 5.570 \\
\hline Women & Mamp-B & Mampi & Mamp 2 & Namp 3 & Mamp 4 & Mame & Mamp 6 & Muno & Mamp 8 & hame & Mamp 10 \\
\hline 1 & 18.724 & 19.307 & 21.712 & 20.554 & 20.412 & 20.387 & 20.335 & 19.178 & 19.062 & 17.416 & 184 \\
\hline 2 & 23.99 & 21.429 & 16.167 & 13.313 & 8.695 & 20.502 & 24.824 & 24.014 & 24.799 & 22.226 & 271 \\
\hline 3 & 23.435 & 14.882 & 21.493 & 25.48 & 25.094 & 23.937 & 21.802 & 21.724 & 23.217 & 23.384 & 3.037 \\
\hline 4 & 15.246 & 16.515 & 17.66 & 17.493 & 16.502 & 16.849 & 16.206 & 16.438 & 16.078 & 19 & 139 \\
\hline 5 & 16.69 & 13.338 & 16.528 & 15.032 & 15.152 & 39 & 13 & 29 & 12.142 & 11.268 & 598 \\
\hline 6 & 20.864 & 17.789 & 21.107 & 24.605 & 21.866 & 24.412 & 23.989 & 23.705 & 23.217 & 22.201 & 1.48 \\
\hline 7 & 21.426 & 19.949 & 22.316 & 22.574 & 19.332 & 19.602 & 19.345 & 9.1 & 19.216 & 19.023 & 19.229 \\
\hline 8 & 18.643 & 15.396 & 18.046 & 16.078 & 14.046 & 13.801 & 13.429 & 827 & 13.145 & 82 & 12 \\
\hline 9 & 16.064 & 16.554 & 17.608 & 17.814 & 18.2 & 18.098 & 17.274 & 17.879 & 18.097 & 18.162 & 84 \\
\hline MEAN & 19.454 & 17.240 & 19.182 & 19.216 & 17.700 & 18.998 & 18.815 & 18.766 & 18.775 & 17.869 & 18.037 \\
\hline SD & 3.169 & 2.613 & 2.436 & 4.308 & 4.804 & 3.907 & 4.434 & 3.945 & 4.465 & 4.422 & 4.644 \\
\hline O-Men & Mamp-B & 1) 2 me & Mamp 2 & Manos & Mamp 4 & Yhano 5 & Mamp 6 & ame & Mamp 8 & Mano & Mamp 10 \\
\hline 1 & 12.693 & 12.232 & 15.409 & 15.165 & 14.586 & 10.046 & 5.521 & 4.423 & 4.116 & 4.232 & 4.309 \\
\hline 2 & 9.564 & 11.512 & 18.509 & 17.235 & 17.814 & 17.017 & 15.949 & 15.512 & 16.065 & 15.1 & 15.1 \\
\hline 3 & 8.504 & 7.048 & 7.974 & 6.675 & 5.904 & 6.045 & 6.02 & 4.219 & 142 & 575 & 3.653 \\
\hline 4 & 15.017 & 10.625 & 8.926 & 11.126 & 9.415 & 10.394 & 10.071 & 9.734 & 9.347 & 11 & 9.749 \\
\hline 5 & 13.88 & 13.737 & 11.821 & 13.968 & 12.901 & 14.971 & 10.097 & 9.904 & 9.763 & 57 & 9.771 \\
\hline 6 & 11.393 & 11.409 & 14.231 & 14.585 & 13.164 & 9.52 & 6.045 & 5.115 & .231 & 308 & 5.231 \\
\hline 7 & 12.821 & 13.158 & 15.126 & 15.885 & 15.923 & 15.307 & 15.255 & 14.226 & 14.355 & 13.338 & 3.698 \\
\hline 8 & 22.895 & 18.735 & 16.128 & 18.109 & 17.87 & 19.791 & 15.178 & 14.366 & 14.408 & 13.176 & 14.751 \\
\hline MEAN & 13.346 & 12.307 & 13.516 & 14.094 & 13.447 & 12.886 & 10.517 & 9.687 & 9.678 & 9.137 & 9.533 \\
\hline SD & 4.416 & 3.295 & 3.647 & 3.673 & 4.133 & 4.588 & 4.460 & 4.705 & 4.877 & 4.486 & 4.721 \\
\hline -Women & Mamp-B & Manes & Wamp 2 & Mano: & Mamp 4 & andioge & Namp 6 & Whanos & Mamp 8 & (2mo: & Vamp 10 \\
\hline 1 & 8.657 & 9.107 & 10.11 & 12.669 & 14.65 & 14.11 & 12.116 & 10.033 & 8.541 & 9.248 & 8.181 \\
\hline 2 & 16.51 & 17.647 & 19.782 & 19.358 & 19.126 & 18.908 & 18.92 & 19.461 & 18.972 & 19.01 & 18.856 \\
\hline 3 & 14.978 & 16.424 & 18.972 & 18.406 & 17.622 & 16.63 & 15.345 & 16.09 & 14.688 & 14.174 & 8.605 \\
\hline 4 & 10.714 & 10.598 & 11.73 & 13.582 & 13.801 & 14.457 & 14.843 & 14.65 & 14.11 & 13.981 & 14.02 \\
\hline 5 & 16.268 & 17.145 & 21.184 & 21.056 & 20.541 & 20.399 & 20.862 & 20.207 & 19.73 & 18.881 & 18.985 \\
\hline 6 & 11.939 & 13.879 & 15.77 & 13.582 & 11.614 & 9.402 & 10.804 & 9.067 & 9.196 & 8.669 & 7.319 \\
\hline 7 & 10.93 & 9.003 & 14.946 & 15.294 & 14.135 & 12.721 & 14.187 & 12.914 & 12.129 & 12.412 & 11.962 \\
\hline 8 & 13.178 & 12.927 & 15.28 & 14.47 & 13.274 & 11.474 & 11.01 & 10.238 & 9.942 & 8.695 & 8.155 \\
\hline 9 & 6.589 & 7.087 & 7.566 & 7.891 & 6.53 & 6.581 & 7.032 & 5.239 & 5.027 & 4.921 & 4.665 \\
\hline MEAN & 12.196 & 12.646 & 15.038 & 15.145 & 14.588 & 13.854 & 13.902 & 13.100 & 12.482 & 12.221 & 11.194 \\
\hline & 86 & 05 & 4.575 & 991 & 4.207 & 18 & 4.258 & 4.975 & 4.882 & .803 & 5.13 \\
\hline
\end{tabular}




\begin{tabular}{|c|c|c|c|c|c|c|c|c|c|c|c|}
\hline & & & & & & & & & & & \\
\hline Y-Men & Marea-B & Sered 1 & Marea 2 & 31832 & Marea 4 & area & Marea 6 & dertest & Marea 8 & 15.tes: & Marea 10 \\
\hline 1 & 0.077 & 0.09 & 0.088 & 0.099 & 0.1 & 0.09 & 0.101 & 0.082 & 0.087 & 0.085 & 0.087 \\
\hline 2 & 0.073 & 0.077 & 0.07 & 0.071 & 0.064 & 0.065 & 0.061 & 0.056 & 0.058 & 0.059 & 0.062 \\
\hline 3 & 0.062 & 0.063 & 0.072 & 0.072 & 0.067 & 0.068 & 0.066 & 0.065 & 0.061 & 0.059 & 0.057 \\
\hline 4 & 0.096 & 0.081 & 0.05 & 0.07 & 0.06 & 0.05 & 0.05 & 0.06 & 0.06 & 0.05 & 0.05 \\
\hline 5 & 0.075 & 0.089 & 0.082 & 0.082 & 0.082 & 0.08 & 0.078 & 0.077 & 0.07 & 0.075 & 0.073 \\
\hline 6 & 0.081 & 0.083 & 0.072 & 0.079 & 0.057 & 0.061 & 0.058 & 0.037 & 0.037 & 0.035 & 0.034 \\
\hline 7 & 0.093 & 0.092 & 0.088 & 0.089 & 0.088 & 0.084 & 0.083 & 0.082 & 0.081 & 0.077 & 0.078 \\
\hline 8 & 0.091 & 0.084 & 0.089 & 0.087 & 0.092 & 0.088 & 0.083 & 0.083 & 0.084 & 0.081 & .083 \\
\hline 9 & 0.079 & 0.068 & 0.045 & 0.035 & 0.051 & 0.029 & 0.025 & 0.044 & 0.046 & 0.058 & 0.057 \\
\hline 10 & 0.069 & 0.074 & 0.07 & 0.076 & 0.072 & 0.071 & 0.07 & 0.074 & 0.073 & 0.073 & 0.073 \\
\hline 11 & 0.083 & 0.087 & 0.095 & 0.097 & 0.095 & 0.099 & 0.091 & 0.088 & 0.09 & 0.09 & 0.09 \\
\hline MEAN & 0.080 & 0.081 & 0.075 & 0.078 & 0.075 & 0.071 & 0.070 & 0.068 & 0.068 & 0.067 & 0.068 \\
\hline SD & 0.010 & 0.009 & 0.016 & 0.017 & 0.017 & 0.020 & 0.021 & 0.017 & 0.017 & 0.017 & 0.017 \\
\hline$Y$-Women & Marea-B & Wreter & Marea 2 & Vlarea & Marea 4 & Mare: & Marea 6 & Mares & Marea 8 & heres & Marea 10 \\
\hline 1 & 0.09 & 0.1 & 0.102 & 0.101 & 0.107 & 0.107 & 0.112 & 0.109 & 0.106 & 0.1 & 0.102 \\
\hline 2 & 0.112 & 0.1 & 0.073 & 0.059 & 0.043 & 0.095 & 0.116 & 0.119 & 0.119 & 0.108 & .124 \\
\hline 3 & 0.088 & 0.07 & 0.077 & 0.087 & 0.089 & 0.082 & 0.076 & 0.073 & 0.081 & 0.08 & 0.081 \\
\hline 4 & 0.097 & 0.105 & 0.101 & 0.104 & 0.094 & 0.094 & 0.102 & 0.093 & 0.122 & 0.099 & .094 \\
\hline 5 & 0.049 & 0.04 & 0.044 & 0.044 & 0.045 & 0.04 & 0.037 & 0.041 & 0.037 & 0.033 & .031 \\
\hline 6 & 0.096 & 0.084 & 0.098 & 0.098 & 0.101 & 0.098 & 0.076 & 0.084 & 0.156 & 0.072 & 0.092 \\
\hline 7 & 0.075 & 0.068 & 0.07 & 0.075 & 0.068 & 0.071 & 0.073 & 0.072 & 0.07 & 0.071 & 0.073 \\
\hline 8 & 0.078 & 0.065 & 0.076 & 0.069 & 0.064 & 0.064 & 0.063 & 0.065 & 058 & 0.055 & 059 \\
\hline 9 & 0.08 & 0.085 & 0.084 & 0.087 & 0.091 & 0.091 & 0.091 & 0.094 & 0.098 & 0.098 & 0.098 \\
\hline MEAN & 0.085 & 0.080 & 0.081 & 0.080 & 0.078 & 0.082 & 0.083 & 0.083 & 0.094 & 0.080 & 0.084 \\
\hline SD & 0.018 & 0.021 & 0.018 & 0.020 & 0.024 & 0.021 & 0.025 & 0.024 & 0.037 & 0.025 & 0.027 \\
\hline O-Men & Marea-B & Meros in & Marea 2 & Mora? & Marea 4 & Wrevers & Marea 6 & 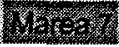 & Marea 8 & 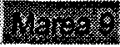 & Mark \\
\hline 1 & 0.052 & 0.052 & 0.057 & 0.06 & 0.06 & 0.048 & 0.03 & 0.022 & 0.023 & 0.025 & 0.028 \\
\hline 2 & 0.038 & 0.044 & 0.069 & 0.064 & 0.072 & 0.067 & 0.066 & 0.071 & 0.074 & 0.063 & 0.062 \\
\hline 3 & 0.042 & 0.033 & 0.037 & 0.031 & 0.031 & 0.03 & 0.031 & 0.02 & 0.022 & 0.018 & 0.021 \\
\hline 4 & 0.047 & 0.034 & 0.03 & 0.035 & 0.025 & 0.03 & 0.031 & 0.035 & 025 & 0.027 & 025 \\
\hline 5 & 0.062 & 0.058 & 0.049 & 0.056 & 0.054 & 0.06 & 0.046 & 0.043 & 0.045 & 0.049 & 0.045 \\
\hline 6 & 0.05 & 0.045 & 0.06 & 0.066 & 0.062 & 0.048 & 0.037 & 0.036 & 0.033 & 0.033 & 0.032 \\
\hline 7 & 0.066 & 0.069 & 0.075 & 0.081 & 0.082 & 0.08 & 0.081 & 0.079 & 0.079 & 0.074 & 0.077 \\
\hline 8 & 0.074 & 0.071 & & 0.076 & 0.076 & 0.08 & 0.069 & 0.071 & 0.06 & 0.06 & 0.071 \\
\hline MEAN & 0.054 & 0.051 & 0.056 & 0.059 & 0.058 & 0.055 & 0.049 & 0.047 & 0.045 & 0.044 & 0.045 \\
\hline SD & 0.012 & 0.014 & 0.016 & 0.018 & 0.021 & 0.020 & 0.020 & 0.023 & 0.023 & 0.021 & 0.022 \\
\hline -Women & Marea-B & 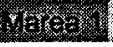 & |Marea 2 & Weterat & Marea 4 & V10 & Marea 6 & 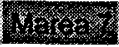 & Marea 8 & Mreres & Marea 10 \\
\hline 1 & 0.036 & 0.035 & 0.041 & 0.054 & 0.061 & 0.059 & 0.05 & 0.042 & 0.034 & 0.038 & 0.034 \\
\hline 2 & 0.07 & 0.072 & 0.077 & 0.077 & 0.077 & 0.077 & 0.083 & 0.082 & 0.086 & 0.085 & 0.084 \\
\hline 3 & 0.096 & 0.103 & 0.109 & 0.113 & 0.116 & 0.112 & 0.114 & 0.118 & 0.125 & 0.12 & 0.087 \\
\hline 4 & 0.053 & 0.055 & 0.047 & 0.053 & 0.054 & 0.056 & 0.055 & 0.056 & 0.058 & 0.059 & 0.059 \\
\hline 5 & 0.071 & 0.067 & 0.08 & 0.082 & 0.083 & 0.083 & 0.086 & 0.085 & 0.084 & 0.087 & 0.087 \\
\hline 6 & 0.068 & 0.054 & 0.066 & 0.052 & 0.047 & 0.033 & 0.04 & 0.033 & 0.034 & 0.036 & 0.029 \\
\hline 7 & 0.036 & 0.026 & 0.043 & 0.046 & 0.046 & 0.039 & 0.047 & 0.046 & 0.043 & 0.044 & 0.042 \\
\hline 8 & 0.048 & 0.048 & 0.053 & 0.05 & 0.049 & 0.044 & 0.042 & 0.039 & 0.039 & 0.035 & 0.033 \\
\hline 9 & 0.035 & 0.041 & 0.042 & 0.044 & 0.037 & 0.04 & 0.041 & 0.034 & 0.03 & 0.027 & 0.025 \\
\hline MEAN & 0.0570 & 0.0557 & 0.0620 & 0.0634 & 0.0633 & 0.0603 & 0.0620 & 0.0594 & 0.0592 & 0.0590 & 0.0533 \\
\hline SD & 0.0208 & 0.0230 & 0.0231 & 0.0228 & 0.0248 & 0.0259 & 0.0261 & 0.0293 & 0.0325 & 0.0316 & 0.0263 \\
\hline
\end{tabular}




\begin{tabular}{|c|c|c|c|c|c|c|c|c|c|c|c|}
\hline Y-Men & Mdur-B & Ware & Mour 2 & 1968: & Mdur 4 & Mañ & Mour 6 & What & Idur 8 & atorg & Mau \\
\hline 1 & 0.018 & 0.0247 & 0.0202 & 0.0236 & 0.0251 & 0.0202 & 0.0247 & 0.0195 & 0.0198 & 0.0191 & 0.0195 \\
\hline 2 & 0.022 & 0.0217 & 0.0183 & 0.0187 & 0.0198 & 0.0202 & 0.0202 & 0.0195 & 0.0202 & 0.0206 & 0.021 \\
\hline 3 & 0.0226 & 0.0228 & 0.0213 & 0.0228 & 0.0213 & 0.0236 & 0.0247 & 0.0258 & 0.0251 & 0.0258 & 0.0255 \\
\hline 4 & 0.0223 & 0.0262 & 0.0187 & 0.0202 & 0.0202 & 0.0206 & 0.0198 & 0.0236 & 0.0217 & 0.0189 & 0.0206 \\
\hline 5 & 0.0203 & 0.0217 & 0.0165 & 0.0202 & 0.0187 & 0.0213 & 0.0202 & 0.0217 & 0.024 & 0.0247 & .0228 \\
\hline 6 & 0.0186 & 0.0183 & 0.0187 & 0.0195 & 0.0202 & 0.0206 & 0.0206 & 0.0228 & 0.0251 & 0.0273 & 0.0288 \\
\hline 7 & 0.0216 & 0.0228 & 0.0191 & 0.0195 & 0.0191 & 0.0195 & 0.0183 & 0.0191 & 0.0187 & 0.0172 & 0.0172 \\
\hline 8 & 0.0253 & 0.0255 & 0.021 & 0.0228 & 0.0236 & 0.0266 & 0.0273 & 0.0285 & 0.0285 & 0.0333 & 0.0348 \\
\hline 9 & 0.0233 & 0.0273 & 0.0277 & 0.0307 & 0.0308 & 0.0374 & 0.03 & 0.0449 & 0.0374 & 0.0363 & 0.0367 \\
\hline 10 & 0.0196 & 0.0236 & 0.0195 & 0.0228 & 0.0213 & 0.0213 & 0.0206 & 0.0213 & 0.0225 & 0.0228 & 0.0236 \\
\hline 11 & 0.0183 & 0.0195 & 0.0195 & 0.0198 & 0.0202 & 0.0213 & 0.0213 & 0.0217 & 0.0221 & 0.0232 & 0.0232 \\
\hline MEAN & 0.0211 & 0.0231 & 0.0200 & 0.0219 & 0.0218 & 0.0230 & 0.0225 & 0.0244 & 0.0241 & 0.0245 & 0.0249 \\
\hline SD & 0.0023 & 0.0028 & 0.0029 & 0.0034 & 0.0035 & 0.0052 & 0.0036 & 0.0074 & 0.0052 & 0.0060 & 0.0062 \\
\hline Y-Women & Mdur-B & Molis: & Mdur 2 & noles & Mour 4 & Dores & Mdur 6 & Whorz & Nour 8 & Moura & Mdur 10 \\
\hline 1 & 0.0246 & 0.0277 & 0.0247 & 0.0255 & 0.0262 & 0.0255 & 0.0258 & 0.0262 & 0.0258 & 0.0273 & 0.0266 \\
\hline 2 & 0.0226 & 0.0225 & 0.0206 & 0.0213 & 0.0206 & 0.0217 & 0.0228 & 0.0247 & 0.0228 & 0.0228 & 0.0243 \\
\hline 3 & 0.0236 & 0.0322 & 0.0206 & 0.021 & 0.0213 & 0.021 & 0.0232 & 0.0206 & 0.0213 & 0.021 & 0.0221 \\
\hline 4 & 0.0359 & 0.0363 & 0.0337 & 0.0348 & 0.0337 & 0.0303 & 0.0382 & 0.0318 & 0.0562 & 0.0442 & 0.0378 \\
\hline 5 & 0.016 & 0.0142 & 0.0135 & 0.0165 & 0.0154 & 0.0142 & 0.0146 & 0.0154 & 0.015 & 0.0146 & 0.0146 \\
\hline 6 & 0.0246 & 0.0266 & 0.0236 & 0.0221 & 0.024 & 0.0232 & 0.0139 & 0.0191 & 0.0524 & 0.015 & 0.0255 \\
\hline 7 & 0.018 & 0.0176 & 0.0161 & 0.0165 & 0.0165 & 0.0172 & 0.018 & 0.0187 & 83 & 0.0191 & 191 \\
\hline 8 & 0.0213 & 0.0221 & 0.0206 & 0.0206 & 0.0213 & 0.0228 & 0.0225 & 0.0236 & 0.0217 & 0.0232 & 0.0228 \\
\hline 9 & 0.024 & 0.0251 & 0.0213 & 0.0221 & 0.0221 & 0.0213 & 0.0217 & 0.0225 & 0.0217 & 0.0221 & 0.0228 \\
\hline MEAN & 0.0234 & 0.0249 & 0.0216 & 0.0223 & 0.0223 & 0.0219 & 0.0223 & 0.0225 & 0.0284 & 0.0233 & 0.0240 \\
\hline SD & 0.0056 & 0.0069 & 0.0057 & 0.0055 & 0.0054 & 0.0046 & 0.0072 & 0.0048 & 0.0150 & 0.0088 & 0.0063 \\
\hline O-Men & Mdur-B & Matir & Mdur 2 & Norrs & Mdur 4 & Mant & Mdur 6 & Wration & Maur 8 & Wours & Mdur 10 \\
\hline 1 & 0.021 & 0.0221 & 0.0191 & 0.0198 & 0.0 & 0.0232 & 0.0262 & 0.0277 & 0.03 & 0.03 & 26 \\
\hline 2 & 0.0186 & 0.0191 & 0.0176 & 0.0168 & 0.0191 & 0.0176 & 0.0191 & 0.021 & 0.0228 & 0.0195 & 0.0183 \\
\hline 3 & 0.023 & 0.0225 & 0.021 & 0.0206 & 0.0217 & 0.0217 & 0.0217 & 0.0206 & 0.0221 & 0.021 & 0.0236 \\
\hline 4 & 0.017 & 0.0165 & 0.0168 & 0.0154 & 0.0161 & 0.0165 & 0.0158 & 0.0202 & 0.0187 & 0.0154 & 0.0161 \\
\hline 5 & 0.022 & 0.0206 & 0.0172 & 0.0195 & 0.0202 & 0.0172 & 0.0213 & 0.0168 & 0.0221 & 0.0292 & 0.0206 \\
\hline 6 & 0.0186 & 0.0221 & 0.0176 & 0.0198 & 0.0191 & 0.0232 & 0.0191 & 0.0277 & 0.0227 & 0.0195 & 0.0183 \\
\hline 7 & 0.0206 & 0.0217 & 0.0198 & 0.0206 & 0.021 & 0.0213 & 0.0221 & 0.0228 & 0.0232 & 0.0221 & 0.0221 \\
\hline 8 & 0.021 & 0.0202 & 0.0195 & 0.0172 & 0.0213 & 0.0168 & 0.0221 & 0.0206 & 0.0187 & 0.0221 & 0.0187 \\
\hline MEAN & 0.0202 & 0.0206 & 0.0186 & 0.0187 & 0.0197 & 0.0197 & 0.0209 & 0.0222 & 0.0225 & 0.0224 & 0.0213 \\
\hline SD & 0.0020 & 0.0020 & 0.0015 & 0.0020 & 0.0018 & 0.0029 & 0.0030 & 0.0038 & 0.0035 & 0.0050 & 0.0052 \\
\hline O-Women & Mdur-B & 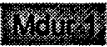 & Maur 2 & Whor & Mdur 4 & 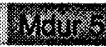 & Mour 6 & Whoriz & Mour 8 & Whating & IMdur 10 \\
\hline 1 & 0.0206 & 0.0191 & 0.0191 & 0.0202 & 0.0198 & 0.0191 & 0.0191 & 0.0195 & 0.0191 & 0.0187 & 0.0191 \\
\hline 2 & 0.019 & 0.0191 & 0.0168 & 0.0168 & 0.0176 & 0.018 & 0.0191 & 0.0191 & 0.0198 & 0.0202 & 0.0202 \\
\hline 3 & 0.0283 & 0.0273 & 0.0247 & 0.0251 & 0.0262 & 0.0281 & 0.03 & 0.0281 & 0.0341 & 0.0356 & 0.0423 \\
\hline 4 & 0.0243 & 0.0277 & 0.0198 & 0.0217 & 0.0206 & 0.021 & 0.0202 & 0.0202 & 0.0228 & 0.0221 & 0.0232 \\
\hline 5 & 0.0236 & 0.0198 & 0.0187 & 0.0183 & 0.0191 & 0.0183 & 0.0187 & 0.0187 & 0.0191 & 0.0206 & 0.0198 \\
\hline 6 & 0.0226 & 0.0183 & 0.021 & 0.0191 & 0.0202 & 0.0191 & 0.0198 & 0.0202 & 0.0206 & 0.021 & 0.0213 \\
\hline 7 & 0.0143 & 0.0109 & 0.0139 & 0.0157 & 0.0161 & 0.0154 & 0.0161 & 0.018 & 0.0191 & 0.0187 & 0.018 \\
\hline 8 & 0.02 & 0.0195 & 0.0168 & 0.0165 & 0.0168 & 0.0183 & 0.0187 & 0.015 & 0.0183 & 0.0198 & 0.0187 \\
\hline 9 & 0.0263 & 0.0281 & 0.0206 & 0.0206 & 0.0187 & 0.0194 & 0.0221 & 0.0225 & 0.0197 & 0.0185 & 0.0206 \\
\hline MEA & 0.0221 & 0.0211 & 0.0190 & 0.0193 & 0.0195 & 0.0196 & 0.0204 & 0.0201 & 0.0214 & 0.0217 & 0.0226 \\
\hline SD & 0.0042 & 0.0056 & 0.0031 & 0.0030 & 0.0030 & 0.0035 & 0.0039 & 0.0036 & 0.0049 & 0.0054 & 0.0076 \\
\hline
\end{tabular}




\begin{tabular}{|c|c|c|c|c|c|c|c|c|c|c|c|}
\hline Men & AEMG B & MS & EMG 2 & EMG & AEMG 4 & $A E$ & IG 6 & & & & \\
\hline 1 & 0.602 & 0.578 & 0.604 & 0.564 & 0.52 & 0.566 & 0.49 & 0.632 & 0.632 & 0.644 & 0.496 \\
\hline 2 & 0.356 & 0.322 & 0.41 & 0.47 & 0.33 & 0.378 & 0.362 & 0.346 & 0.348 & 0.35 & 0.282 \\
\hline 3 & 0.476 & 0.482 & 0.456 & 0.522 & 0.408 & 0.456 & 0.526 & 0.352 & 0.364 & 0.392 & 0.268 \\
\hline 4 & 0.688 & 0.74 & 0.58 & 0.64 & 0.44 & 0.34 & 0.44 & 0.424 & 0.413 & 0.4176 & 0.4206 \\
\hline 5 & 0.382 & 0.28 & 0.296 & 0.244 & 0.278 & 0.268 & 0.318 & 0.214 & 0.298 & 0.266 & 0.28 \\
\hline 6 & 0.512 & 0.446 & 0.52 & 0.382 & 0.394 & 0.468 & 0.506 & 0.388 & 0.372 & 0.33 & 0.42 \\
\hline 7 & 0.464 & 0.386 & 0.452 & 0.42 & 0.364 & 0.304 & 0.374 & 0.438 & 0.314 & 0.326 & 0.554 \\
\hline 8 & 0.662 & 0.57 & 0.778 & 0.62 & 0.698 & 0.5 & 0.528 & 0.482 & 0.398 & 0.412 & 0.442 \\
\hline 9 & 0.552 & 0.502 & 0.668 & 0.614 & 0.688 & 0.694 & 0.578 & 0.552 & 0.526 & 0.698 & 0.72 \\
\hline 10 & 0.518 & 0.554 & 0.476 & 0.456 & 0.45 & 0.396 & 0.426 & 0.374 & 0.378 & 0.308 & 0.346 \\
\hline 11 & 0.696 & 0.734 & 0.656 & 0.64 & 0.49 & 0.588 & 0.46 & 0.462 & 0.5 & 0.45 & 0.398 \\
\hline MEAN & 0.537 & 0.509 & 0.536 & 0.507 & 0.460 & 0.451 & 0.455 & 0.424 & 0.413 & 0.418 & 0.421 \\
\hline SD & 0.116 & 0.149 & 0.137 & 0.126 & 0.134 & 0.130 & 0.080 & 0.111 & 0.101 & 0.137 & 0.135 \\
\hline Y-Women & AEMG B & AEMG 1 & AEMG 2 & AEMG 3 & AEMG 4 & AEMG 5 & AEMG 6 & AEMG 7 & AEMG 8 & AEMG 9 & AEMG10 \\
\hline 1 & 0.964 & 0.848 & 0.908 & 1.092 & 0.676 & 0.88 & 0.81 & 0.948 & 0.468 & 0.49 & 1.052 \\
\hline 2 & 0.964 & 0.534 & 1.262 & 1.544 & 0.766 & 0.992 & 0.578 & 1.048 & 0.868 & 0.798 & 0.654 \\
\hline 3 & 0.67 & 0.482 & 0.524 & 0.388 & 0.354 & 0.29 & 0.34 & 0.278 & 0.27 & 0.29 & 0.27 \\
\hline 4 & 0.312 & 0.342 & 0.292 & 0.328 & 0.298 & 0.346 & 0.308 & 0.3 & 0.34 & 0.338 & 0.322 \\
\hline 5 & 0.46 & 0.398 & 0.426 & 0.382 & 0.308 & 0.32 & 0.298 & 0.326 & 0.308 & 0.344 & 0.322 \\
\hline 6 & 0.392 & 0.422 & 0.388 & 0.556 & 0.394 & 0.572 & 0.326 & 0.282 & 0.35 & 0.35 & 0.274 \\
\hline 7 & 0.408 & 0.39 & 0.388 & 0.48 & 0.506 & 0.606 & 0.506 & 0.574 & 0.564 & 0.55 & 0.4 \\
\hline 8 & 0.432 & 0.398 & 0.392 & 0.498 & 0.51 & 0.454 & 0.436 & 0.438 & 0.364 & 0.41 & 0.466 \\
\hline 9 & 0.656 & 0.604 & 0.558 & 0.774 & 0.858 & 0.788 & 0.604 & 0.812 & 0.892 & 0.742 & 0.656 \\
\hline MEAN & 0.584 & 0.491 & 0.571 & 0.671 & 0.519 & 0.583 & 0.467 & 0.556 & 0.492 & 0.479 & 0.491 \\
\hline SD & 0.245 & 0.157 & 0.315 & 0.405 & 0.205 & 0.256 & 0.174 & 0.305 & 0.237 & 0.184 & 0.258 \\
\hline O-Men & AEMG B & AEMG 1 & AEMG 2 & AEMG 3 & AEMG 4 & AEMG 5 & AEMG 6 & AEMG 7 & AEMG 8 & AEMG 9 & AEMG10 \\
\hline 1 & 0.558 & 0.562 & 0.552 & 0.47 & 0.486 & 0.472 & 0.564 & 0.378 & 0.406 & 0.42 & 0.358 \\
\hline 2 & 0.304 & 0.324 & 0.342 & 0.258 & 0.286 & 0.282 & 0.256 & 0.322 & 0.288 & 0.302 & 0.274 \\
\hline 3 & 0.264 & 0.294 & 0.284 & 0.29 & 0.212 & 0.37 & 0.324 & 0.344 & 0.318 & 0.34 & 0.306 \\
\hline 4 & 0.378 & 0.316 & 0.32 & 0.318 & 0.248 & 0.394 & 0.388 & 0.23 & 0.364 & 0.266 & 0.262 \\
\hline 5 & 0.49 & 0.398 & 0.33 & 0.436 & 0.398 & 0.346 & 0.448 & 0.386 & 0.478 & 0.446 & 0.354 \\
\hline 6 & 0.42 & 0.416 & 0.358 & 0.45 & 0.336 & 0.402 & 0.394 & 0.368 & 0.364 & 0.398 & 0.37 \\
\hline 7 & 0.334 & 0.18 & 0.256 & 0.214 & 0.24 & 0.312 & 0.23 & 0.238 & 0.23 & 0.24 & 0.252 \\
\hline 8 & 0.462 & 0.44 & 0.422 & 0.482 & 0.474 & 0.444 & 0.418 & 0.422 & 0.398 & 0.414 & 0.428 \\
\hline MEAN & 0.401 & 0.366 & 0.358 & 0.365 & 0.335 & 0.378 & 0.378 & 0.336 & 0.356 & 0.353 & 0.326 \\
\hline SD & 0.100 & 0.114 & 0.093 & 0.106 & 0.107 & 0.064 & 0.108 & 0.069 & 0.077 & 0.077 & 0.062 \\
\hline
\end{tabular}

O-Women AEMG B AEMG 1 AEMG 2 AEMG 3 AEMG 4 AEMG 5 AEMG 6 AEMG 7 AEMG 8 AEMG 9 AEMG10

$\begin{array}{cccccccccccc}1 & 0.394 & 0.298 & 0.346 & 0.394 & 0.398 & 0.448 & 0.578 & 0.42 & 0.658 & 0.564 & 0.476 \\ 2 & 0.636 & 0.594 & 0.654 & 0.63 & 0.728 & 0.702 & 0.71 & 0.688 & 0.75 & 0.582 & 0.674 \\ 3 & 0.928 & 0.824 & 0.876 & 0.902 & 1.008 & 0.86 & 1.05 & 0.798 & 1.016 & 0.772 & 0.968 \\ 4 & 0.196 & 0.194 & 0.35 & 0.184 & 0.308 & 0.278 & 0.294 & 0.308 & 0.36 & 0.324 & 0.37 \\ 5 & 0.924 & 0.624 & 0.542 & 0.696 & 0.738 & 0.628 & 0.644 & 0.428 & 0.642 & 0.6 & 0.6 \\ 6 & 0.472 & 0.484 & 0.51 & 0.53 & 0.478 & 0.446 & 0.514 & 0.576 & 0.484 & 0.508 & 0.466 \\ 7 & 0.366 & 0.31 & 0.328 & 0.426 & 0.352 & 0.416 & 0.3 & 0.376 & 0.376 & 0.35 & 0.32 \\ 8 & 0.378 & 0.46 & 0.45 & 0.494 & 0.444 & 0.462 & 0.39 & 0.398 & 0.476 & 0.392 & 0.408 \\ 9 & 0.29 & 0.3 & 0.346 & 0.284 & 0.238 & 0.182 & 0.228 & 0.234 & 0.216 & 0.206 & 0.28 \\ \text { MEAN } & 0.509 & 0.454 & 0.489 & 0.504 & 0.521 & 0.491 & 0.523 & 0.470 & 0.553 & 0.478 & 0.507 \\ \text { SD } & 0.265 & 0.201 & 0.183 & 0.218 & 0.251 & 0.209 & 0.259 & 0.183 & 0.241 & 0.174 & 0.214\end{array}$




\begin{tabular}{|c|c|c|c|c|c|c|c|c|c|c|c|}
\hline \multicolumn{12}{|l|}{$82915 T$} \\
\hline er & MUAB & $m$ & & & & & & & & & \\
\hline 1 & 96.12 & 90.53 & 86.93 & 84.71 & 84.30 & 91.11 & 62.80 & 7.00 & 83.44 & 60.83 & 70.75 \\
\hline 2 & 65.38 & 76.63 & 71.63 & 87.70 & 77.22 & 75.11 & 71.22 & 89.02 & 62.54 & 67.76 & 49.03 \\
\hline 3 & 93.78 & 88.88 & 96.34 & 96.65 & 78.12 & 85.34 & 66.47 & 87.50 & 56.25 & 73.50 & 78.22 \\
\hline 4 & 100.00 & 100.00 & 91.29 & 100.00 & 100.00 & 48.64 & 89.89 & 78.13 & 71.04 & 14.98 & 73.71 \\
\hline 5 & 92.54 & 88.65 & 93.84 & 95.32 & 84.27 & 83.09 & 79.44 & 68.36 & 53.70 & 77.94 & 76.12 \\
\hline 6 & 94.62 & 43.78 & -3.20 & 49.51 & 44.21 & 34.73 & 30.49 & 29.85 & 22.93 & 27.87 & -0.64 \\
\hline 7 & 98.94 & 85.58 & 90.40 & 91.43 & 89.39 & 88.90 & 67.84 & 86.60 & 75.80 & 68.18 & 84.31 \\
\hline 8 & 62.23 & 68.47 & 74.09 & 56.02 & 56.79 & 51.58 & 28.96 & 56.43 & 38.61 & 45.15 & 49.81 \\
\hline 9 & 100.00 & 92.95 & 84.16 & 79.19 & 75.08 & 97.71 & 97.89 & 77.16 & 74.01 & 47.63 & 90.57 \\
\hline 10 & 100.00 & 96.65 & 98.91 & 100.00 & 98.05 & 100.00 & 73.43 & 97.48 & 100.00 & 100.00 & 100.00 \\
\hline 11 & 91.03 & 91.13 & 92.23 & 80.97 & 80.21 & 64.99 & 65.85 & 70.51 & 64.48 & 63.10 & 60.00 \\
\hline 12 & 94.76 & 100.00 & 81.56 & 81.99 & 77.85 & 71.60 & 61.62 & 46.20 & 60.44 & 1.45 & 1.45 \\
\hline MEAN & 90.78 & 85.27 & 79.85 & 83.62 & 78.79 & 74.40 & 66.32 & 72.85 & 63.60 & 59.03 & 66.11 \\
\hline SD & 12.98 & 15.92 & 27.46 & 16.17 & 15.65 & 20.76 & 20.26 & 19.94 & 20.17 & 22.69 & 26.07 \\
\hline Woms & MUA B & MUA 1 & MUA2 & MOA 3. & MUA4 & MUAS & MUA 6 & MUA7 & MUA 8 & MUA 9 & MUA \\
\hline 1 & 88.47 & 92.81 & 85.52 & 80.15 & 69.69 & 59.22 & 55.56 & 30.53 & 80.32 & 34.39 & \\
\hline 2 & 80.34 & 99.18 & 88.78 & 100.00 & 91.35 & 86.21 & 83.10 & 67.14 & 82.01 & & \\
\hline 3 & 82.81 & 80.93 & 74.91 & & 79.62 & 93.09 & 68.39 & 80.50 & 63.55 & .15 & 60.64 \\
\hline 4 & 57.22 & 77.55 & 86.47 & 86.79 & 84.97 & 74.21 & 86.94 & 71.04 & 74.28 & 0.79 & 9.83 \\
\hline 5 & 88.96 & 91.60 & 73.10 & 85.76 & 82.73 & 79.39 & 78.54 & 75.38 & 72.78 & 2.78 & 34 \\
\hline 6 & 76.83 & 62.29 & 55.50 & 47.22 & 50.53 & 54.30 & 44.74 & 28.72 & 37.63 & 2.05 & 40.17 \\
\hline 7 & 89.36 & 65.73 & 77.92 & 88.8 & 69.68 & 64.45 & 73.97 & 69.62 & 56.97 & 77 & 63.17 \\
\hline 8 & 88.29 & 71.99 & 66.84 & 88.62 & 88.04 & 87.07 & 65.27 & 87.83 & 100.00 & .86 & 3.44 \\
\hline 9 & 67.56 & 78.43 & 84.39 & 68.87 & 66.37 & 64.03 & 62.35 & 67.13 & 37.07 & .10 & 3.54 \\
\hline MEAN & 79.98 & 80.06 & 77.05 & 81.72 & 75.89 & 73 & 76 & 21 & 18 & 21 & 58.61 \\
\hline SD & 11.18 & 12.53 & 10.85 & 15.36 & 12.94 & 13.73 & 13.53 & 20.72 & 20.76 & 21.82 & 17.90 \\
\hline Me & & & & MUA 3 & & & MUA 6 & MUAT & MUA 8 & MUA 9 & MUA 10 \\
\hline 1 & 73.44 & 87.02 & 82.77 & 80.78 & 69.49 & & 92.01 & 82.80 & & 55 & 00 \\
\hline 2 & 95.82 & 89.64 & 97.49 & 93. & 77.27 & 82 & 35 & 66.80 & 51.51 & 88 & \\
\hline 3 & 80.65 & 64.26 & 88.13 & 85.07 & 84.35 & 83.48 & 86.36 & 77.94 & 83.37 & 70 & 1 \\
\hline 4 & 79.43 & 62.90 & 79.96 & & 57.91 & & & & & & 11 \\
\hline 5 & 30 & 84 & 84 & 86 & 82.53 & 70 & 39 & 30 & 8 & 50 & 4 \\
\hline 6 & 95.83 & 77.43 & 82.68 & 94.25 & 69.18 & 86.73 & 72.59 & 71.91 & 75.29 & 10 & 5 \\
\hline 7 & 90.00 & & 73 & & 78.06 & & 60 & & & & 0 \\
\hline 8 & 30 & & 77 & & & & & & & & 33 \\
\hline 9 & 95.68 & 39.88 & 55.30 & 46 & 44.33 & 60.44 & 23.00 & -20.24 & -16.18 & -29.91 & 14.32 \\
\hline 10 & & & 84.64 & & & & & & & 19 & 72.69 \\
\hline 11 & & & 80 & & & & & & & & 18 \\
\hline 12 & 71.27 & 75.44 & 80.20 & 92 & 82.06 & 57 & 64.11 & 77.99 & 34 & 6.32 & 70.53 \\
\hline MEAN & & 74.61 & 80.56 & & 74.09 & & & 63.14 & & 8.57 & 67.15 \\
\hline $\mathrm{s}$ & & 13.73 & 9.96 & & 13.02 & & & 29.69 & & 33.87 & \\
\hline Wom & MUAB & MUA 1 & MUA 2 & MUA3 & MUA 4 & MUA 5 & MUA6 & MUA7 & MUA 8 & MUA & MU \\
\hline 1 & 86.81 & 80.47 & 72.77 & 85.52 & 52.47 & 64.71 & 60.91 & 39.66 & 59.85 & 48.24 & 64.25 \\
\hline 2 & & & 53 & & & & & & & 28 & \\
\hline 3 & & & 6 & & & & & & & & \\
\hline 4 & 94.02 & 86.01 & 96.36 & 79.03 & 76.94 & 70.34 & 66.97 & 57.53 & 63.73 & 62.37 & 56.98 \\
\hline 5 & 100.00 & 99.29 & 100.00 & 85.29 & 88.37 & 87.85 & 86.95 & 90.73 & 88.39 & 90.60 & 77.42 \\
\hline 6 & & 67. & 84 & & & & & & & 65 & \\
\hline 7 & 75.56 & 56.11 & & & 65.07 & 58.29 & 46 & & & 63 & 38 \\
\hline 8 & 82.78 & 68.32 & 65.42 & 71. & 69.08 & 71.82 & 72.15 & 55.99 & 91 & 33 & 57.65 \\
\hline 9 & 22 & 73.89 & 81. & & & & & & & 93 & \\
\hline 10 & & 9 & 70. & & & & & & 21 & 24 & \\
\hline 11 & 8 & 77.05 & 92.69 & 95. & 86.74 & 80.76 & 92.36 & 77.99 & 81.51 & 3.54 & 39.05 \\
\hline $\mathrm{ME}$ & & 71.82 & 72.13 & 74. & 71.24 & 70.94 & 69.27 & 61.63 & 66.59 & 5.00 & 63.15 \\
\hline $\mathrm{SL}$ & 13.51 & 14.26 & 25.05 & 15.67 & 14.78 & 16.55 & 17.32 & 28.34 & 19.00 & 20.58 & 19.89 \\
\hline
\end{tabular}




\begin{tabular}{|c|c|c|c|c|}
\hline Y-Men & & PT 1: & PT 10 & FIPT \\
\hline & 1 & 0.887 & 0.906 & -2.14205 \\
\hline & 2 & 1.01 & 0.718 & 28.91089 \\
\hline & 3 & 1.304 & 0.528 & 59.5092 \\
\hline & 4 & 0.55 & 0.563 & -2.36364 \\
\hline & 5 & 0.934 & 0.49 & 47.53747 \\
\hline & 6 & 0.957 & 0.626 & 34.58725 \\
\hline & 7 & 0.832 & 0.274 & 67.06731 \\
\hline & 8 & 1.202 & 1.036 & 13.81032 \\
\hline & 9 & 0.893 & 0.594 & 33.48264 \\
\hline & 10 & 0.925 & 0.2 & 78.37838 \\
\hline & 11 & 1.128 & 0.62 & 45.03546 \\
\hline & 12 & 1.313 & 0.747 & 43.10739 \\
\hline MEAN & & 0.994583 & 0.6085 & 37.24339 \\
\hline SD & & 0.216184 & 0.234412 & 25.29473 \\
\hline
\end{tabular}

\begin{tabular}{|c|c|c|c|c|}
\hline O-Men & \multicolumn{2}{|c|}{ PT 1} & \multicolumn{2}{|c|}{ FI PT } \\
\hline & 1 & 0.709 & 0.183 & 74.189 \\
\hline & 2 & 1.516 & 0.763 & 49.67018 \\
\hline & 3 & 0.652 & 0.34 & 47.85276 \\
\hline & 4 & 0.779 & 0.709 & 8.985879 \\
\hline & 5 & 0.766 & 0.651 & 15.01305 \\
\hline & 6 & 0.7 & 0.401 & 42.71429 \\
\hline & 7 & 0.385 & 0.391 & -1.55844 \\
\hline & 8 & 0.607 & 0.547 & 9.884679 \\
\hline & 9 & 0.677 & 0.391 & 42.2452 \\
\hline & 0 & 0.687 & 0.791 & -15.1383 \\
\hline & 1 & 0.604 & 0.544 & 9.933775 \\
\hline & 2 & 1.205 & 0.811 & 32.6971 \\
\hline MEAN & & 0.773917 & 0.5435 & 26.3741 \\
\hline SD & & 0.299407 & 0.204082 & 25.80749 \\
\hline
\end{tabular}

\begin{tabular}{|c|c|c|c|}
\hline Y-Women & T 1 & PT 10 & FI PT \\
\hline 1 & 1.071 & 0.302 & 71.80205 \\
\hline 2 & 0.607 & 0.346 & 42.99835 \\
\hline 3 & 0.677 & 0.499 & 26.29247 \\
\hline 4 & 0.747 & 0.41 & 45.11379 \\
\hline 5 & 0.69 & 0.397 & 42.46377 \\
\hline 6 & 0.734 & 0.47 & 35.9673 \\
\hline 7 & 0.588 & 0.346 & 41.15646 \\
\hline 8 & 0.702 & 0.582 & 17.09402 \\
\hline 9 & 0.83 & 0.702 & 15.42169 \\
\hline 10 & 0.429 & 0.391 & 8.857809 \\
\hline 11 & 0.664 & 0.448 & 32.53012 \\
\hline 12 & 1.224 & 0.505 & 58.74183 \\
\hline MEAN & 0.746917 & 0.449833 & 36.53664 \\
\hline SD & 0.213761 & 0.112081 & 18.1403 \\
\hline
\end{tabular}

\begin{tabular}{lrrrr}
\multicolumn{2}{c}{ O-Women PT } & PT 10 & FI PT \\
\hline & 1 & 0.435 & 0.642 & -47.5862 \\
& 2 & 0.594 & 0.352 & 40.74074 \\
& 3 & 0.407 & 0.248 & 39.06634 \\
& 4 & 0.372 & 0.259 & 30.37634 \\
& 5 & 0.722 & 0.258 & 64.26593 \\
& 6 & 0.423 & 0.175 & 58.62884 \\
& 7 & 0.362 & 0.41 & -13.2597 \\
& 8 & 0.499 & 0.394 & 21.04208 \\
& 9 & 0.524 & 0.392 & 25.19084 \\
& 10 & 0.302 & 0.582 & -92.7152 \\
& 11 & 0.575 & 0.436 & 24.17391 \\
MEAN & 12 & 0.645 & 0.379 & 41.24031 \\
SD & 0.488333 & 0.37725 & 15.93035 \\
& 0.126903 & 0.136012 & 45.75599
\end{tabular}


FIMVE

\begin{tabular}{rrrrrr} 
Y-Men & \multicolumn{3}{c}{ MVC B } & MVC 10 & F MVC \\
\hline & 1 & 6.86 & 4.43 & 35.42274 \\
& 2 & 12.76 & 8.4 & 34.16928 \\
& 3 & 14.03 & 5.14 & 63.36422 \\
& 4 & 8.73 & 5.4 & 38.14433 \\
& 5 & 12.71 & 6.48 & 49.01652 \\
& 6 & 5.32 & 2.59 & 51.31579 \\
& 7 & 11.98 & 7.27 & 39.31553 \\
& 8 & 13.17 & 6.59 & 49.96203 \\
& 9 & 8.88 & 5.82 & 34.45946 \\
& 10 & 9.53 & 5.08 & 46.69465 \\
& 11 & 7.9 & 4.29 & 45.6962 \\
& 12 & 10.65 & 4.19 & 60.65728 \\
MEAN & 10.21 & 5.473333 & 45.68484 \\
SD & 2.768744 & 1.563634 & 9.817478
\end{tabular}

O-Men MVC B MVC 10 FIMVC

$\begin{array}{llll}1 & 8.85 & 5.92 & 33.10734\end{array}$

$\begin{array}{llll}2 & 7.6 & 5.12 & 32.63158\end{array}$

$\begin{array}{lrrr}3 & 6.81 & 4.5 & 33.9207\end{array}$

$\begin{array}{llll}4 & 6.47 & 5.78 & 10.66461\end{array}$

$\begin{array}{llll}5 & 7.38 & 7.65 & -3.65854\end{array}$

$\begin{array}{llll}6 & 6.39 & 4.09 & 35.99374\end{array}$

$\begin{array}{llll}7 & 4.54 & 3.5 & 22.90749\end{array}$

$\begin{array}{llll}8 & 8.52 & 8.03 & 5.751174\end{array}$

$\begin{array}{llll}9 & 6.1 & 5.42 & 11.14754\end{array}$

$\begin{array}{llll}10 & 5.37 & 4.62 & 13.96648\end{array}$

$\begin{array}{llll}11 & 6.18 & 4.54 & 26.53722\end{array}$

$\begin{array}{llll}12 & 9.53 & 6.98 & 26.75761\end{array}$

$\begin{array}{llll}\text { MEAN } & 6.978333 & 5.5125 & 20.81058\end{array}$

$\begin{array}{lllll}\text { SD } & 1.462021 & 1.423875 & 12.91436\end{array}$
Y-Women MVC B MVC 10 FI MVC

$\begin{array}{lrrrr} & 1 & 8.36 & 3.42 & 59.09091 \\ & 2 & 5.27 & 4.12 & 21.82163 \\ & 3 & 7.24 & 4.27 & 41.0221 \\ & 4 & 6.51 & 4.42 & 32.10445 \\ & 5 & 7.68 & 4.74 & 38.28125 \\ & 6 & 7.28 & 3.12 & 57.14286 \\ & 7 & 7.08 & 3.84 & 45.76271 \\ & 8 & 7.24 & 6.23 & 13.95028 \\ & 9 & 5.58 & 2.66 & 52.32975 \\ & 10 & 5.28 & 3.81 & 27.84091 \\ & 11 & 4.93 & 3.58 & 27.38337 \\ \text { MEAN } & 12 & 6.32 & 3.96 & 37.34177 \\ \text { SD } & 6.564167 & 4.014167 & 37.83933 \\ & & 1.094934 & 0.900903 & 14.06808\end{array}$

O-Women MVC B MVC 10 FIMVC

$\begin{array}{lrrrr} & 1 & 7.75 & 5.95 & 23.22581 \\ & 2 & 6.65 & 4.98 & 25.11278 \\ & 3 & 6.39 & 5.03 & 21.28326 \\ & 4 & 3.63 & 4.21 & -15.978 \\ & 5 & 7.25 & 4.13 & 43.03448 \\ & 6 & 6.27 & 4.49 & 28.38915 \\ & 7 & 4.43 & 3.97 & 10.38375 \\ & 8 & 4.65 & 3.6 & 22.58065 \\ & 9 & 7.67 & 5.85 & 23.72881 \\ & 10 & 5.02 & 4.25 & 15.33865 \\ & 11 & 6.14 & 5.83 & 5.04886 \\ & 12 & 6.05 & 2.77 & 54.21488 \\ \text { MEAN } & 5.991667 & 4.588333 & 21.36359 \\ \text { SD } & 1.312436 & 0.976029 & 17.68466\end{array}$




\begin{tabular}{|c|c|c|c|c|c|c|c|c|c|c|c|}
\hline & $t o B$ & Rati & & 3 & 3 Ratio 4 & I Ratio 5 & Ratio 6 & 3 Ratio 7 & rat & 3 & Ratio 1 \\
\hline 1 & 7.818 & 6.422 & 6.864 & 5.697 & 5.200 & 6.289 & 4.851 & 7.707 & 7.264 & 7.576 & 5.701 \\
\hline 2 & 4.877 & 4.182 & 5.857 & 6.620 & 5.156 & 5.815 & 5.934 & 6.179 & 000 & 5.932 & 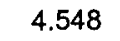 \\
\hline 3 & 7.677 & 7.651 & 6.333 & 7.250 & 6.090 & 6.706 & 7.970 & 5.415 & 5.967 & 6.644 & 4.702 \\
\hline 4 & 7.167 & 9.136 & 11.600 & 9.143 & 7.333 & 800 & .800 & 067 & 83 & & 2 \\
\hline 5 & 5.093 & 3.146 & 3.610 & 2.976 & 3.390 & 3.350 & 4.077 & 2.779 & 257 & 3.547 & .836 \\
\hline 6 & 6.321 & 5.373 & 7.222 & 4.835 & 6.912 & 7.672 & 8.724 & 10.486 & 10.054 & 9.429 & 12.353 \\
\hline 7 & 4.989 & 4.196 & 5.136 & 4.719 & 4.136 & 3.619 & 4.506 & 5.341 & 3.877 & 4.234 & 103 \\
\hline 8 & 7.275 & 6.786 & 8.742 & 7.126 & 7.587 & 5.682 & 6.361 & 5.807 & .738 & 5.086 & 325 \\
\hline 9 & 6.987 & 7.382 & 14.844 & 17.543 & 13.490 & 23.931 & 23.120 & 12.545 & 11.435 & 12.034 & 12.632 \\
\hline 10 & 7.507 & 7.486 & 6.800 & 6.000 & 6.250 & 5.577 & 6.086 & 5.054 & 5.178 & 4.219 & 4.740 \\
\hline 11 & 8.386 & 8.437 & 6.905 & 6.598 & 5.158 & 5.939 & 5.055 & 5.250 & 5.556 & 5.000 & 422 \\
\hline MEAN & 6.736 & 6.382 & 7.629 & 7.137 & 6.428 & 7.398 & 7.771 & 6.694 & 6.474 & 6.550 & 6.707 \\
\hline SD & 1.237 & 1.921 & 3.134 & 3.804 & 2.676 & 5.631 & 5.348 & 2.728 & 2.364 & 2.597 & 3.146 \\
\hline
\end{tabular}

Y-Women Ratio B Ratio 1 Ratio 2 Ratio 3 Ratio 4 Ratio 5 Ratio 6 Ratio 7 Ratio 8 Ratio 9 Ratio 10

$\begin{array}{lllllllllllll}1 & 10.711 & 8.480 & 8.902 & 10.812 & 6.318 & 8.224 & 7.232 & 8.697 & 4.415 & 4.900 & 10.314\end{array}$

$\begin{array}{lllllllllllll}2 & 8.607 & 5.340 & 17.288 & 26.169 & 17.814 & 10.442 & 4.983 & 8.807 & 7.294 & 7.389 & 5.274\end{array}$

$\begin{array}{lllllllllllll}3 & 7.614 & 6.886 & 6.805 & 4.460 & 3.978 & 3.537 & 4.474 & 3.808 & 3.333 & 3.625 & 3.333\end{array}$

$\begin{array}{llllllllllll}4 & 3.216 & 3.257 & 2.891 & 3.154 & 3.170 & 3.681 & 3.020 & 3.226 & 2.787 & 3.414 & 3.426\end{array}$

$\begin{array}{lllllllllllll}5 & 9.388 & 9.950 & 9.682 & 8.682 & 6.844 & 8.000 & 8.054 & 7.951 & 8.324 & 10.424 & 10.387\end{array}$

$\begin{array}{lllllllllllll}6 & 4.083 & 5.024 & 3.959 & 5.673 & 3.901 & 5.837 & 4.289 & 3.357 & 2.244 & 4.861 & 2.978\end{array}$

$\begin{array}{lllllllllllll}7 & 5.440 & 5.735 & 5.543 & 6.400 & 7.441 & 8.535 & 6.932 & 7.972 & 8.057 & 7.746 & 5.479\end{array}$

$\begin{array}{lllllllllllll}8 & 5.538 & 6.123 & 5.158 & 7.217 & 7.969 & 7.094 & 6.921 & 6.738 & 6.276 & 7.455 & 7.898\end{array}$

$\begin{array}{llllllllllll}9 & 8.200 & 7.106 & 6.643 & 8.897 & 9.429 & 8.659 & 6.637 & 8.638 & 9.102 & 7.571 & 6.694\end{array}$

$\begin{array}{lllllllllllll}\text { MEAN } & 6.978 & 6.433 & 7.430 & 9.052 & 7.429 & 7.112 & 5.838 & 6.577 & 5.759 & 6.376 & 6.198\end{array}$

$\begin{array}{lllllllllllll}\text { SD } & 2.531 & 1.971 & 4.284 & 6.837 & 4.419 & 2.338 & 1.688 & 2.420 & 2.611 & 2.310 & 2.856\end{array}$

0-Men Ratio B Ratio 1 Ratio 2 Ratio 3 Ratio 4 Ratio 5 Ratio 6 Ratio 7 Ratio 8 Ratio 9 Ratio 10

$\begin{array}{lllllllllllll}1 & 10.731 & 10.808 & 9.684 & 7.833 & 8.100 & 9.833 & 18.800 & 17.182 & 17.652 & 16.800 & 12.786\end{array}$

$\begin{array}{lllllllllllll}2 & 8.000 & 7.364 & 4.957 & 4.031 & 3.972 & 4.209 & 3.879 & 4.535 & 3.892 & 4.794 & 4.419\end{array}$

$\begin{array}{llllllllllll}3 & 6.286 & 8.909 & 7.676 & 9.355 & 6.839 & 12.333 & 10.452 & 17.200 & 14.455 & 18.889 & 14.571\end{array}$

$\begin{array}{lllllllllllll}4 & 8.043 & 9.294 & 10.667 & 9.086 & 9.920 & 13.133 & 12.516 & 6.571 & 14.560 & 9.852 & 10.480\end{array}$

$\begin{array}{lllllllllllll}5 & 7.903 & 6.862 & 6.735 & 7.786 & 7.370 & 5.767 & 9.739 & 8.977 & 10.622 & 9.102 & 7.867\end{array}$

$\begin{array}{lllllllllllll}6 & 8.400 & 9.244 & 5.967 & 6.818 & 5.419 & 8.375 & 10.649 & 10.222 & 11.030 & 12.061 & 11.563\end{array}$

$\begin{array}{llllllllllll}7 & 5.061 & 2.609 & 3.413 & 2.642 & 2.927 & 3.900 & 2.840 & 3.013 & 2.911 & 3.243 & 3.273\end{array}$

$\begin{array}{lllllllllllll}8 & 6.243 & 6.197 & 6.029 & 6.342 & 6.237 & 5.550 & 6.058 & 5.944 & 6.633 & 6.900 & 6.028\end{array}$

$\begin{array}{llllllllllll}\text { MEAN } & 7.583 & 7.661 & 6.891 & 6.737 & 6.348 & 7.888 & 9.366 & 9.205 & 10.219 & 10.205 & 8.873\end{array}$

$\begin{array}{lllllllllllll}\text { SD } & 1.728 & 2.536 & 2.394 & 2.358 & 2.247 & 3.600 & 5.148 & 5.430 & 5.334 & 5.508 & 4.104\end{array}$

O-Women Rato B Ratio 1 Ratio 2 Rato 3 Ratio 4 Ratio 5 Ratio 6 Ratio 7 Rato 8 Ratio 9 Ratio 10

$\begin{array}{cccccccccccc}1 & 10.944 & 8.514 & 8.439 & 7.296 & 6.525 & 7.593 & 11.560 & 10.000 & 19.353 & 14.842 & 14.000 \\ 2 & 9.086 & 8.250 & 8.494 & 8.182 & 9.455 & 9.117 & 8.554 & 8.390 & 8.721 & 6.847 & 8.024 \\ 3 & 9.667 & 8.000 & 8.037 & 7.982 & 8.690 & 7.679 & 9.211 & 6.763 & 8.128 & 6.433 & 11.126 \\ 4 & 3.698 & 3.527 & 7.447 & 3.472 & 5.704 & 4.964 & 5.345 & 5.500 & 6.207 & 5.492 & 6.271 \\ 5 & 13.014 & 9.313 & 6.775 & 8.488 & 8.892 & 7.566 & 7.488 & 5.035 & 7.643 & 6.897 & 6.897 \\ 6 & 6.941 & 8.963 & 7.727 & 10.192 & 10.170 & 13.515 & 12.850 & 17.455 & 14.235 & 14.111 & 16.069 \\ 7 & 10.167 & 11.923 & 7.628 & 9.261 & 7.652 & 10.667 & 6.383 & 8.174 & 8.744 & 7.955 & 7.619 \\ 8 & 7.875 & 9.583 & 8.491 & 9.880 & 9.061 & 10.500 & 9.286 & 10.205 & 12.205 & 11.200 & 12.364 \\ 9 & 8.286 & 7.317 & 8.238 & 6.455 & 6.432 & 4.550 & 5.561 & 6.882 & 7.200 & 7.630 & 11.200 \\ \text { MEAN } & 8.853 & 8.377 & 7.919 & 7.912 & 8.064 & 8.461 & 8.471 & 8.712 & 10.271 & 9.045 & 10.397 \\ \text { SD } & 2.637 & 2.238 & 0.581 & 2.047 & 1.550 & 2.844 & 2.586 & 3.737 & 4.244 & 3.464 & 3.406\end{array}$




\begin{tabular}{|c|c|c|}
\hline \multicolumn{2}{|c|}{ 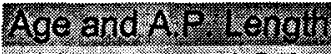 } & \\
\hline Y-Men & AGE & A.P. \\
\hline 1 & 26 & 4.2 \\
\hline 2 & 24 & 4.3 \\
\hline 3 & 26 & 5.5 \\
\hline 4 & 24 & 3.8 \\
\hline 5 & 25 & 3.9 \\
\hline 6 & 27 & 3.6 \\
\hline 7 & 24 & 4 \\
\hline 8 & 24 & 3.8 \\
\hline 9 & 28 & 4.2 \\
\hline 10 & 30 & 4.6 \\
\hline 11 & 23 & 3.3 \\
\hline 12 & 23 & 4.1 \\
\hline MEAN & 25.33 & 4.11 \\
\hline SD & 2.15 & 0.56 \\
\hline O-Men & AGE & A.P. \\
\hline 1 & 61 & 3.7 \\
\hline 2 & 74 & 3.3 \\
\hline 3 & 76 & 3.5 \\
\hline 4 & 77 & 3.6 \\
\hline 5 & 77 & 3.6 \\
\hline 6 & 69 & 2.8 \\
\hline 7 & 74 & 4.2 \\
\hline 8 & 68 & 4.2 \\
\hline 9 & 62 & 4.3 \\
\hline 10 & 75 & 3.8 \\
\hline 11 & 76 & 3.5 \\
\hline 12 & 71 & 4 \\
\hline MEAN & 71.67 & 3.71 \\
\hline SD & 5.60 & 0.43 \\
\hline
\end{tabular}

\begin{tabular}{ccc} 
Y.Women & AGE & A.P. \\
\hline 1 & 24 & 3.5 \\
2 & 21 & 4.6 \\
3 & 24 & 3.3 \\
4 & 27 & 4 \\
5 & 24 & 3.8 \\
6 & 23 & 3.9 \\
7 & 22 & 3.6 \\
8 & 21 & 4 \\
9 & 26 & 3.7 \\
10 & 21 & 3.5 \\
11 & 24 & 3.7 \\
12 & 26 & 3.6 \\
MEAN & 23.58 & 3.77 \\
SD & 2.07 & 0.34
\end{tabular}

$\begin{array}{ccc}\text { O-Women } & \text { AGE } & \text { AP } \\ 1 & 77 & 3.8 \\ 2 & 65 & 3.6 \\ 3 & 68 & 3.5 \\ 4 & 77 & 3.3 \\ 5 & 68 & 3.5 \\ 6 & 74 & 3.6 \\ 7 & 65 & 3.6 \\ 8 & 67 & 3.9 \\ 9 & 72 & 3 \\ 10 & 70 & 3.4 \\ 11 & 63 & 3.1 \\ 12 & 68 & 3.1 \\ \text { MEAN } & 69.50 & 3.45 \\ \text { SD } & 4.62 & 0.28\end{array}$

